\title{
䨔 American Psychological Association
}

2 http://www.apa.org/pubs/journals/rev/

3 "This article may not exactly replicate the final version published in the APA journal. It is not

4 the copy of record."

5 
The complementary roles of auditory and motor information evaluated in a Bayesian perceptuo-motor model of speech perception Raphaël Laurent, Marie-Lou Barnaud, Jean-Luc Schwartz

$$
\text { GIPSA-Lab }
$$

Univ. Grenoble Alpes, GIPSA-Lab, F-38000 Grenoble, France CNRS, GIPSA-Lab, F-38000 Grenoble, France

$$
\text { Pierre Bessière }
$$

CNRS, Sorbonne Universités - Université Pierre et Marie Curie Institut des Systèmes Intelligents et de Robotique, Paris, France

$$
\text { Julien Diard }
$$

Laboratoire de Psychologie et NeuroCognition Univ. Grenoble Alpes, LPNC, F-38000 Grenoble, France CNRS, LPNC, F-38000 Grenoble, France

Authors' Note

The research leading to these results received funding from the European Research Council under the European Community's Seventh Framework Programme (FP7/2007-2013 Grant Agreement no. 339152, "Speech Unit(e)s”, J.-L. Schwartz PI). This work includes research conducted as part of Raphaël Laurent's PhD thesis, defended at the Universite de Grenoble on October, 8th, 2014 (manuscript in French: "COSMO : un modèle bayésien des interactions sensori-motrices dans la perception de la parole"). Portions of this work were also presented at the 9th International Seminar on Speech Production (ISSP 11) in Montréal, Canada (June 20-23, 2011), at the 14th Annual Conference of the International Speech Communication Association (Interspeech 2013) in Lyon, France (August 25-29, 2013) and at the Workshop on Infant Language Development (WILD 15) in Stockholm, 
32 Sweden (June 10-12, 2015). The authors wish to thank Clément Moulin-Frier and Louis-

33 Jean Boë for support and inspiration. Correspondence concerning this paper should be

34 addressed to J.-L. Schwartz, Université Grenoble Alpes, GIPSA-Lab, F-38000 Grenoble,

35 France; CNRS, GIPSA-Lab, F-38000 Grenoble, France; 11 Rue des Mathématiques, 38400

36 Saint-Martin-d'Hères, France. Email: Jean-Luc.Schwartz@gipsa-lab.grenoble-inp.fr, Phone:

$37 \quad(+33) 4765747$ 12, Fax: (+33)4 76574710. 
Abstract

There is a consensus concerning the view that both auditory and motor representations intervene in the perceptual processing of speech units. However, the question of the functional role of each of these systems remains seldom addressed and poorly understood. We capitalized on the formal framework of Bayesian Programming to develop COSMO (Communicating Objects using Sensory-Motor Operations), an integrative model that allows principled comparisons of purely motor or purely auditory implementations of a speech perception task and tests the gain of efficiency provided by their Bayesian fusion. Here, we show three main results. (i) In a set of precisely defined "perfect conditions", auditory and motor theories of speech perception are indistinguishable. (ii) When a learning process that mimics speech development is introduced into COSMO, it departs from these perfect conditions. Then auditory recognition becomes more efficient than motor recognition in dealing with learned stimuli, while motor recognition is more efficient in adverse conditions. We interpret this result as a general "auditory-narrowband vs. motor-wideband" property. (iii) Simulations of plosive-vowel syllable recognition reveal possible cues from motor recognition for the invariant specification of the place of plosive articulation in context, that are lacking in the auditory pathway. This provides COSMO with a second property, where auditory cues would be more efficient for vowel decoding and motor cues for plosive articulation decoding. These simulations provide several predictions, which are in good agreement with experimental data and suggest that there is natural complementarity between auditory and motor processing within a perceptuo-motor theory of speech perception. Keywords: Speech perception, computational modeling, sensory-motor interactions, adverse conditions, plosive invariance 
The complementary roles of auditory and motor information evaluated in a Bayesian perceptuo-motor model of speech perception

\title{
On the functional role of auditory vs. motor systems in speech perception
}

\author{
Since the introduction in the 1960s of the so-called Motor Theory of Speech
}

Perception (Liberman, Cooper, Shankweiler, \& Studdert-Kennedy, 1967), it is striking to remark how the debate pertaining to auditory and motor theories of speech communication has evolved. There were basically two main periods of reasoning.

The arguments from the 1960s to the 1980s mainly derived from experimental phonetics and what would now be called laboratory phonology. These were basically focused on functional questions. Auditory and motor theories were discussed according to their respective abilities to deal with the question of invariance (see an extensive review by Perkell $\&$ Klatt, 1986). Invariants were thought to exist somewhere in the acoustic signal, providing a key for abstract and categorical phonologic units from the continuous and physical substance of phonetics. The debate concerned the nature of these invariants, be this auditory or motor (see reviews of functional arguments in favor of auditory theories e.g. Diehl, Lotto, \& Holt, 2004; Kingston \& Diehl, 1994; Kluender 1994; Lotto 2000; Massaro \& Oden 1980; Nearey, 1990; or in favor of motor invariance in Liberman et al., 1967; Liberman \& Mattingly, 1985, 1989; Liberman \& Whalen, 2000; and a review in Galantucci, Fowler, \& Turvey, 2006). Since the 1990s, the arguments have evolved progressively towards experimental data provided by cognitive neuroscience. With the discovery of mirror neurons (Rizzolatti, Fadiga, Gallese, \& Fogassi, 1996a) and the proposal of a "mirror system" in the human perception of complex actions (Grafton, Arbib, Fadiga, \& Rizzolatti, 1996; Iacoboni et al., 1999; Rizzolatti et al., 1996b), neurophysiological and behavioral experimental data made it progressively clear that the motor system plays a role in speech perception (see a recent detailed review in 
Skipper, Devlin \& Lametti, 2017). Evidence emerged in two steps. Firstly, neuroanatomical studies repeatedly showed that parietal and frontal brain areas associated with speech production were consistently stimulated upon speech perception tasks (e.g. Fadiga, Craighero, Buccino, \& Rizzolatti, 2002; Pulvermüller et al., 2006; Watkins, Strafella, \& Paus, 2003; Wilson, Saygin, Sereno, \& Iacoboni, 2004). This was particularly shown in non-standard conditions involving noise (Binder, Liebenthal, Possing, Medler, \&Ward, 2004; Zekveld, Heslenfeld, Festen, \& Schoonhoven, 2006), non-native stimuli (Callan, Callan, \& Jones, 2014; Callan, Jones, Callan, \& Akahane-Yamada, 2004; Wilson \& Iacoboni, 2006), or conflicting audiovisual inputs (Jones \& Callan, 2003; Ojanen et al., 2005; Skipper, van Wassenhove, Nusbaum, \& Small, 2007). Secondly, behavioral studies looked for a causal role of motor areas in speech perception by altering or modulating the potential efficiency of speech motor centers, by Transcranial Magnetic Stimulation (TMS), repeated TMS or motor perturbations. Such studies have shown small but consistent perceptual effects in categorization or discrimination of speech stimuli, in ambiguous or noisy conditions (e.g., d'Ausilio et al., 2009; d'Ausilio, Bufalari, Salmas, \& Fadiga ,2012; Grabski, Tremblay, Gracco, Girin, \& Sato, 2013; Ito, Tiede, \& Ostry, 2009; Meister, Wilson, Deblieck, Wu, \& Iacoboni, 2007; Möttönen, Dutton, \& Watkins, 2013; Möttönen \& Watkins, 2009; Rogers, Möttönen, Boyles, \& Watkins, 2014; Sato, Tremblay, \& Gracco, 2009; Sato et al., 2011; Shiller, Sato, Gracco, \& Baum, 2009). In this context, the strong "auditory" vs. "motor" controversy about invariance at the crossroads of phonetics and phonology that prevailed until the end of the 1980s was almost completely replaced since the beginning of the 1990s by an integrative view from cognitive neuroscience, assuming that the motor and auditory systems collaborate in speech perception. This has the merits of taking into account new experimental insights, but its drawback is that the question of the respective functions of sensory and motor systems has almost completely 
111 disappeared from the literature. However, if both auditory and motor processes do intervene

112 in speech perception ${ }^{(1)}$, the potential specificity and complementarity of these two systems

113 within a perceptuo-motor speech perception architecture becomes essential. How could it be

114 useful for speech perception to capitalize on two different systems? How could the motor

115 system be more helpful in adverse conditions? What specific aspects of computation, for what

116 kind of information extraction, are respectively implemented by the motor and auditory (if not

117 visual or somatosensory) components of the speech perception system?

118 These are the questions we address in the theoretical framework of the "Perception-

119 for-Action-Control Theory" (PACT). PACT is a perceptuo-motor theory of speech perception,

120 connecting perceptual shaping and motor procedural knowledge in a principled way, in

121 speech multisensory processing within the human brain (Schwartz, Basirat, Ménard, \& Sato,

122 2012a; Schwartz, Boë, \& Abry, 2007). PACT considers that perceptual knowledge is involved

123 in both speech comprehension and speech control, in a communicative process. The

124 communication unit through which parity may be achieved, is neither a sound, nor a gesture,

125 but a perceptually-shaped gesture, that is a perceptuo-motor unit characterized both by its

126 articulatory coherence, provided by its gestural nature and its perceptual value, necessary for

127 function. Motor processes could be associated with multisensory processes through audio-

128 visuo-motor binding, enabling a better extraction of adequate cues for further categorization

129 processes (Basirat, Schwartz, \& Sato, 2012; see also Skipper, van Wassenhove, Nusbaum \&

130 Small, 2007). Furthermore, perceptual categorization would benefit from motor information

131 in addition to auditory and possibly visual clues. This would, improve variability processing

132 and the extraction of invariance (Schwartz, Abry, Boë, \& Cathiard, 2002; Schwartz et al.,

$1332007,2012 a)$.

134 In PACT, it is also acknowledged that perception and action are co-structured in the

135 course of speech development, which involves both producing and perceiving speech items. 
136 The schedule of perceptuo-motor development in the first few years of age is important in this

137 context, and seems to incorporate several major steps (Kuhl, 2004; Kuhl et al., 2008). First,

138 auditory processes mature, enabling categorization of many phonetic contrasts almost from

139 birth (e.g., Bertoncini, Bijeljac-Babic, Blumstein, \& Mehler, 1987; Eimas, Siqueland,

140 Jusczyk, \& Vigorito, 1971; Jusczyk \& Derrah, 1987), with an early focus on the sounds of the

141 infant's language. This can be as early as 6 months old for vowels (Kuhl, Williams, Lacerda,

142 Stevens, \& Lindblom, 1992) and 10 months old for consonants (Werker \& Tees, 1984). Motor

143 processes evolve later and more slowly, beginning by articulatory exploration of the possible

144 vocal repertoire, with canonical babbling at around 7 months of age (Davis, MacNeilage, \&

145 Matyear, 2002; MacNeilage, 1998). This continues with a later focus on the sounds of the

146 phonological system from the end of the first year and through the following ones.

147 Importantly, canonical babbling, sometimes considered as a purely endogenous process

148 enabling infants to extensively explore the possibilities of their vocal tracts, seems to be

149 influenced since its very beginning by the language heard in the surrounding environment.

150 Such "babbling drift" has been displayed in a number of experiments concerning vowel

151 formants, consonant-vowel associations and prosodic schemes (e. g. de Boysson-Bardies,

152 1993; de Boysson-Bardies, Hallé, Sagart, \& Durant, 1989; de Boysson-Bardies, Sagart, \&

153 Durant, 1984).

154 Auditory perception is hence mature and focused before orofacial control occurs.

155 Furthermore, the connection between the speech perception system and the motor system

156 through the parieto-frontal dorsal pathway in the cortex does not seem to be completely

157 mature at birth, but rather evolves throughout the first year of life (Dehaene-Lambertz,

158 Dehaene, \& Hertz-Pannier, 2002; Dehaene-Lambertz et al., 2006; vs. Kuhl, Ramírez,

159 Bosseler, Lotus Lin, \& Imada, 2014; Imada et al., 2006). Consequently, motor information

160 would not be mature, focused or fully available for perception until the end of the first year. 


\section{Computational models of auditory vs. motor theories of speech perception}

In the context of debates concerning the potential role of auditory and motor systems

There are already many computational models of auditory theories of speech perception.

167 Many, if not all of acoustic speech recognition systems can be construed as such, as they involve the best statistical analyses of the acoustic content of large speech corpora for speech understanding (see recent reviews in e.g. Hinton et al., 2012; Huang \& Deng, 2010). They also often incorporate more or less sophisticated computational models of the auditory analysis of acoustic stimuli in the human brain (e.g. Hermansky, 1998; Deng, 1999). Auditory theory models also include computational psycholinguistic models of cognitive speech processing (e.g. Trace: McClelland \& Elman, 1986; the Distributed Cohort Model: Gaskell \&

A widespread mathematical framework, in this domain, is probabilistic modeling, where a generative, predictive model associates probable acoustic signals with linguistic categories. Then, perception is cast as a categorization process, in which Bayes theorem is used to infer the most likely linguistic category given some acoustic stimulus:

$$
P\left(\left[O=o_{i}\right] \mid S\right)=\frac{P\left(S \mid\left[O=o_{i}\right]\right) P\left(\left[O=o_{i}\right]\right)}{\sum_{j} P\left(S \mid\left[O=o_{j}\right]\right) P\left(\left[O=o_{j}\right]\right)},
$$

181 where $P\left(S \mid\left[O=o_{i}\right]\right)$ expresses the probability distribution of acoustic cues for a given

182 category and $P\left(\left[O=o_{i}\right]\right)$ defines prior probabilities of each category.

183 The origin of such models can be traced back, historically, to Signal Detection Theory

184 (Tanner \& Swets, 1954; Green \& Swets, 1966; more recent references include Dayan \& 
Recognition Theory (Ashby \& Townsend, 1986; Ashby \& Perrin, 1988). Recent years saw the resurgence and spread of Bayesian models of speech perception, that consider the categorization process above to model the optimal, ideal acoustic (or possibly visual, see footnote 1) information processing system, without any reference to motor processes. Such “optimal” models include ideal listener models (Feldman, Griffiths \& Morgan, 2009;

Sonderegger \& Yu, 2010) and ideal adapter models (Clayards, Aslin, Tanenhaus \& Jacobs, 2007; Clayards, Tanenhaus, Aslin, \& Jacobs, 2008; Kleinschmidt \& Jaeger, 2011, 2015), where categories are either learned in a batch manner (De Boer \& Kuhl, 2003; Dillon, Dunbar \& Idsardi, 2013) or acquired and adapted incrementally (McMurray, Aslin \& Toscano, 2009; Vallabha, McClelland, Pons, Werker \& Amano, 2007). In this trend, many extensions were proposed, for instance for dealing with multiple cues (Toscano \& McMurray, 2008, 2010) or with higher-level structures above phonemic representations (e.g., words, syllables), in hierarchical models (Norris \& McQueen, 2008; Feldman, Griffiths \& Morgan, 2009b; Kiebel, Daunizeau, \& Friston, 2009; Feldman, Griffiths, Goldwater \& Morgan, 2013).

In comparison, there are many less computational motor theory models, basically because of the lack of easily available articulatory or motor data required for the training of such models. A few automatic speech recognition systems attempt to introduce articulatory data into their statistical processes (e.g. Deng \& Ma, 2000; Deng, Ramsay, \& Sun, 1997; Frankel, Richmond, King \& Taylor, 2000; Sun \& Deng, 2002) and a recent series of machine learning models based on artificial neural networks and applied to articulatory data recorded through electromagnetic articulography aimed to show the efficiency of articulatory inputs for phonetic decoding (e.g. Canevari, Badino, d'Ausilio, Fadiga, \& Metta, 2013; Castellini et al., 2011). A few variants of Bayesian models of speech perception, because they consider computations of the speaker's intentions through motor inversion, can be construed as involving motor knowledge during perception, although it was not their initial purpose, as 
211 they were developed instead to model perceptual magnet effects (Feldman \& Griffiths, 2007;

212 Feldman, Griffiths \& Morgan, 2009). Other authors attempted to develop formal models

213 without real articulatory ground truth data to evaluate the possibility of implementing motor

214 or perceptuo-motor theories of speech perception (e.g. Kröger, Kannampuzha, \& Kaufmann,

215 2014; Kröger, Kannampuzha, \& Neuschaefer-Rube, 2009; Moore, 2007).

216 However, while all these developments basically aim to demonstrate that articulatory

217 or motor speech decoding is indeed feasible and potentially efficient, none of this research

218 attempts to really evaluate why and how motor information could be relevant for speech

219 decoding. Furthermore, it is always difficult in these models to precisely disentangle what

220 comes from the distribution of articulatory information and what comes from specific choices

221 in the computational implementation.

222 This is why the Bayesian implementation of an instance of motor theory

223 (implementing motor decoding in a Bayesian framework) or perceptuo-motor theory

224 (including the fusion of auditory and motor information) could enable the functional role of

225 the motor system to be assessed more clearly and rigorously. This is the objective of the

226 COSMO model (Communicating Objects using Sensory-Motor Operations) that will be 227 presented in the next section.

COSMO, a Bayesian computational framework for assessing the functional role of

auditory vs. motor systems

To attempt to better understand the function of motor information in speech

232 perception, we have developed over recent years a computational Bayesian framework called

233 COSMO. This model enables auditory, motor and perceptuo-motor theories of speech

234 communication to be implemented and compared in a coherent set of simple probabilistic

235 equations and processes, based on Bayesian modeling. COSMO was initially developed to 
deal with the emergence of sound systems in human languages (Moulin-Frier, Diard, Schwartz, \& Bessière, 2015; Moulin-Frier, Schwartz, Diard, \& Bessière, 2011) and was then adapted to the study of speech perception in adverse conditions (Moulin-Frier, Laurent, Bessière, Schwartz, \& Diard, 2012). The present article greatly expands the initial study of Moulin-Frier et al. (2012), which attempted to clearly assess when and how motor information could be useful for phonetic decoding.

A first part will present the COSMO model, together with an initial crucial result we obtained with COSMO, which we called the indistinguishability theorem. This theorem shows that in a set of precisely defined "perfect conditions", auditory and motor theories of speech perception are indistinguishable (Moulin-Frier et al., 2012). We will present this theorem in detail, since it is of great theoretical importance, providing a landmark for any further comparison of auditory and motor models of speech perception.

Indeed, distinguishing the functional roles of auditory and motor systems for speech perception can only be achieved by departing from these perfect conditions. This can occur in one of two major ways, providing the two major contributions of the present paper to the subject.

Firstly, the learning process can differentiate the auditory and motor systems. We claim that these two systems evolve differently during learning. The auditory system could focus rapidly and precisely on the set of learning stimuli provided by the environment, leading to a system finely tuned to this learning set. This would provide the auditory system with a "narrow-band" specificity with respect to the learning data. In contrast, the motor system would "wander" more through the sensory-motor space during its exploration stage, because of the complexity of the task at hand. Hence it would evolve more slowly and focus less efficiently on the set of learning stimuli provided by the environment, in agreement with the developmental timeline described previously. However, it would be able to process a wider 
set of stimuli thanks to the "wandering" phenomenon. This would provide the motor system with a "wide-band" specificity, making it poorer for learned stimuli, but better at generalizing about adverse conditions involving unlearned stimuli. This will be developed in Part 2, together with two predictions associated with this "auditory-narrow, motor-wide" property, that will be compared to the available experimental data.

Secondly, the two systems can be differentiated in terms of the nature and complexity of their internal representations, possibly leading to different processing of variability of the phonological units. Considering simulations of the recognition of plosive-vowel sequences, we explore the assumption that motor recognition might provide clues as to the invariant specification of the place of articulation of plosives in context, which is lacking in the auditory pathway, while the auditory categorization of vowels would be more straightforward than its motor counterpart. Altogether, this suggests that there should be a natural complementarity between auditory and motor systems within a perceptuo-motor theory of speech communication. This will be developed in Part 3, together with two other predictions that will be discussed in light of available experimental data.

Following the important simulation contributions and predictions, we will end this paper with a review of some major perspectives and challenges associated with the development of COSMO, in relation to cognitive processes involved in speech communication.

\section{Part 1 - COSMO and the indistinguishability theorem}

In this first part we will introduce the two major pieces of our computational framework.

Firstly, we will present and describe COSMO together with its mathematical specification and the way it enables modeling of auditory, motor or perceptuo-motor theories of speech perception. Secondly, we will derive the indistinguishability theorem, already published by 
Moulin-Frier et al. (2012), but which will be explained more precisely in the present paper.

This will provide us with the crucial landmark that will serve for further simulations presented in parts 2 and 3.

\section{The COSMO model}

COSMO stems from the analysis of spoken communication, which can be broken down into a minimal set of variables, with a high level of abstraction.

***FIGURE 1 ABOUT HERE***

As is shown in the upper part of Figure 1, to communicate about an object $O_{S}$, the Speaker Agent performs a motor gesture $M$ resulting in a sensory input $S$ from which the Listener Agent retrieves the object $O_{L}{ }^{(2)}$. The variable $C^{E n v}$ is a Boolean variable assessing the communication success: it is True when $O_{S}=O_{L}$.

Our work is based on the hypothesis that the Communicating Agent, is able to act both as a speaker and a listener, and has internal representations of the whole communication loop, as shown in the lower part of Figure 1. Consequently, the Communicating Agent model is made up of (i) a motor system associating motor representations $M$ to the object $O_{S}$ to be produced and of (ii) a sensory system associating the perceived object $O_{L}$ to the sensory representation $S$, which are linked by (iii) a sensory-motor system that allows the consequences of motor commands $M$ in terms of sensory inputs $S$ to be predicted. At this stage, $M, S$ and $O$ are still generic variables, in order not to lose generality. They will be instantiated for experiments and made more precise later in this paper.

The model contains two different variables $O_{S}$ and $O_{L}$, one for the intention of the speaker, the other for the perception of the listener. They are also useful to avoid a directed loop from a single variable $O$ to itself through motor and perceptual variables $M$ and $S$. Indeed, such loops are not compatible with straightforward application of Bayes theorem; 
312 duplication of the variables is a classic solution to circumvent this technical problem (e.g.

313 state variables are replicated over time for temporal series models). Such duplication between

314 phonological codes for production and perception, linked by conversion mechanisms, is

315 compatible with neuropsychological data (Jacquemot, Dupoux, \& Bachoud-Lévi, 2007).

316 Coherence between variables $O_{S}$ and $O_{L}$ is imposed by (iv) a Boolean variable $C$ when it is set

317 to True. The variable $C$ can also be conceived as the internalization of the $C^{E n v}$ variable

318 assessing communication success.

319 This Communicating Agent model has a name, COSMO, that also happens to recall

320 the model variables. COSMO is formally defined within the framework of Bayesian

321 Programming (Bessière, Laugier, \& Siegwart, 2008; Bessière, Mazer, Ahuactzin-Larios, \&

322 Mekhnacha, 2013; Lebeltel, Bessière, Diard, \& Mazer, 2004) by probability distributions,

323 which encode the subjective knowledge that the agent has about the relations between its

324 internal representations. The COSMO model is thus defined by one mathematical object, the

325 joint probability distribution over all its variables: $P\left(C O_{L} S M O_{S}\right)$. It contains all the

326 information the agent has about its internal variables and can be shown to be sufficient to

327 perform any inference task about these variables, whatever the form. In other words, any

328 conditional probability over some of these variables, knowing some others, can be computed

329 from the joint probability distribution. We chose to decompose and simplify this joint

330 probability distribution as follows (Moulin-Frier et al., 2012):

$$
P\left(C O_{L} S M O_{S}\right)=\underbrace{P\left(O_{S}\right)}_{\text {prior }} \underbrace{P\left(M \mid O_{S}\right)}_{\begin{array}{c}
\text { motor } \\
\text { repertoire }
\end{array}} \underbrace{P(S \mid M)}_{\begin{array}{c}
\text { forward } \\
\text { model }
\end{array}} \underbrace{P\left(O_{L} \mid S\right)}_{\begin{array}{c}
\text { sensory } \\
\text { classifier }
\end{array}} \underbrace{P\left(C \mid O_{S} O_{L}\right)}_{\begin{array}{c}
\text { communication } \\
\text { success }
\end{array}} \cdot(1)
$$

Various tasks can then be carried out by asking questions to the model, by computing

333 conditional probability distributions of the form P(SEARCHED |OBSERVATIONS): What is

334 the probability distribution over the SEARCHED variables, knowing the value of some

335 OBSERVATIONS? In the COSMO framework, a speech production task amounts to 
computing a conditional distribution of the form $P(M \mid O)$ : What is the probability distribution over motor commands $M$ corresponding to the object $O$ to be communicated? A speech perception task amounts to computing a conditional distribution of the form $P(O \mid S)$ : What is the probability distribution over perceived objects $O$, given the sensory input $S$ ? Within the framework of COSMO, these questions can be instantiated in three different ways: (i) by replacing $O$ by $O_{S}$ we implement a motor theory focused on the speaker's perspective, (ii) by replacing $O$ by $O_{L}$ we implement an auditory theory focused on the listener's perspective, (iii) by indifferently using either $O_{S}$ or $O_{L}$ and by further conditioning the computed distribution with the constraint $C=$ True, we implement a perceptuo-motor theory that ensures the coherence of both representations. This is the equivalent of explicitly setting the communication success as a goal of the task considered. Bayesian inference provides a way to compute the conditional probability distributions corresponding to all these tasks from the joint probability distribution that defines the COSMO model (Equation (1)). Figure 2 shows the results of these computations and how they can be interpreted. We now explain further the results of these Bayesian inferences, focusing on the speech perception task.

\section{***FIGURE 2 ABOUT HERE***}

As can be seen in Figure 2, the implementation of an auditory theory of perception consists of a direct computation of $P(O \mid S)=P\left(O_{L} \mid S\right)$, with no intervention of motor variables. This is consistent with classical proposals about auditory theories, which deny the role of motor knowledge in speech perception and consider that it is based exclusively on the set of auditory processing and categorization mechanisms available in the human brain (e.g. Diehl et al., 2004). We note that this portion of our model is equivalent to many preceding models of acoustic categorization, including the Ideal Adapter model of Kleinschmidt \& Jaeger (2015) mentioned previously. 
Likewise, the implementation of a motor theory of perception, i.e., computing

$P(O \mid S)=P\left(O_{S} \mid S\right) \propto \sum_{M}\left(P\left(M \mid O_{S}\right) P(S \mid M)\right)$ (Feldman \& Griffiths, 2007; Feldman,

Griffiths \& Morgan, 2009; Moulin-Frier et al., 2012), is consistent with the view that speech

perception occurs by retrieving the intended motor gestures of the speaker (Liberman \&

$S$ through an inverse model, which is followed by the categorization process estimating $O_{S}$

from $M$ through a motor decoder. In the Bayesian framework, these are replaced by the computation of the sum over the possible values of the variable $M$, weighted by the probability that they have the sensory consequence $S$ and by the probability that they are associated with $O_{S}$ the considered object. This is a Bayesian analogue to analysis-by-synthesis (Halle \& Stevens, 1959; Stevens \& Halle, 1967; see a review in Bever \& Poeppel, 2010). The deterministic two-stage process, firstly with motor-to-sensory inversion and secondly with motor decoding, is an approximation of the summation over all $M$ values.

376 of a mere Bayesian fusion of the predictions of the sensory and motor categorization

377 processes: $P([O=o] \mid S[C=$ True $]) \propto P\left(\left[O_{L}=o\right] \mid S\right) \sum_{M}\left(P\left(M \mid\left[O_{S}=o\right]\right) P(S \mid M)\right)$.

Indistinguishability of auditory and motor theories in perfect conditions of learning and communication

Although purely sensory and purely motor perceptions are described by different

382 equations (see Figure 2), it can be proven that if three hypotheses defining a set of "perfect 383 conditions" of learning are verified, the motor and auditory theories of perception make 384 exactly the same predictions. Therefore, these cannot be distinguished empirically. This demonstration has been presented previously (Moulin-Frier et al., 2012), but in a less explicit 
formulation. We will present it here again in detail, in its more rigorous form, with three hypotheses instead of the two used previously.

\section{***FIGURE 3 ABOUT HERE***}

We consider a supervised learning scenario, shown in Figure 3, which features

Learning Agents and a Master Agent, each described as a COSMO agent. To distinguish their variables, superscripts are added and variables become $O_{S}^{A g}, O_{S}^{\text {Master }}, M^{A g}, M^{\text {Master }}$, etc. In the learning scenario, the Learning Agent is provided by the Master Agent with the following < object, stimulus> pairs. The Master Agent uniformly selects $O_{S}^{\text {Master }}$ objects, draws corresponding $M^{\text {Master }}$ motor commands according to the production model $P\left(M^{\text {Master }} \mid O_{S}^{\text {Master }}\right)$, which are then transformed by the environment modeled by $P\left(S^{A g} \mid M^{\text {Master }}\right)$ and result in sensory $S^{A g}$ inputs. Furthermore, the variable $C^{E n v}$, which ensures coherence between the $O_{S}^{\text {Master }}$ and $O_{L}^{A g}$ objects, implements a shared attention mechanism, e.g. deixis, which allows the Learning Agent to retrieve the right objects $\left(O_{L}^{A g}\right)$ from the Master to associate with the $S^{A g}$ stimuli in its sensory classifiers $P\left(O_{L}^{A g} \mid S^{A g}\right)$. The Learning Agent builds its sensory classifier through successive random draws, which are mathematically expressed by the following approximation:

$P\left(O_{L}^{A g} \mid S^{A g}\right) \approx \sum_{M}\left(P\left(M^{\text {Master }} \mid O_{S}^{\text {Master }}\right) . P\left(S^{A g} \mid M^{\text {Master }}\right)\right)$.

In this equation, the sign $\approx$ expresses the fact that the set of learning stimuli (right part of the equation) has to be learned in some way from the $P\left(O_{L}^{A g} \mid S^{A g}\right)$ distribution (left part of the equation).

We now define the three hypotheses used in this approach and prove that their conjunction ensures the indistinguishability of the motor and auditory theories of speech perception.

i. H1 (the "perfect sensory learning hypothesis"): the sensory classifier is 
perfectly learned from the Master's productions, i.e. $P\left(O_{L}^{A g} \mid S^{A g}\right)=$ $\sum_{M}\left(P\left(M^{\text {Master }} \mid O_{S}^{\text {Master }}\right) \cdot P\left(S^{A g} \mid M^{\text {Master }}\right)\right)$. By replacing the operator $\approx$ of Equation (2) by an equality operator =, H1 explicitly states that the sensory classifier $P\left(O_{L}^{A g} \mid S^{A g}\right)$ learned by the agent perfectly encodes all the information expressed by the combination of the probability distributions $P\left(M^{\text {Master }} \mid O_{S}^{\text {Master }}\right)$ and $P\left(S^{A g} \mid M^{\text {Master }}\right)$. These describe the way the Master performs its motor gestures and the way they are transformed by the environment.

ii. H2 (the "perfect motor learning hypothesis"): the motor repertoire of the agent is identical to that of the Master, i.e. $P\left(M^{A g} \mid O_{S}^{A g}\right)=P\left(M^{\text {Master }} \mid O_{S}^{\text {Master }}\right)$. iii. H3 (the "perfect sensory-motor learning hypothesis"): the agent's sensorymotor system perfectly encodes the properties of the transformation performed by the environment during the learning process, i.e. $P\left(S^{A g} \mid M^{A g}\right)=$ $P\left(S^{A g} \mid M^{\text {Master }}\right)$.

The indistinguishability theorem states that if $\mathrm{H} 1, \mathrm{H} 2$ and $\mathrm{H} 3$ hold, then the motor and sensory instantiations of the speech perception task are indistinguishable.

The proof is straightforward. Starting from Equation (2), which states how the sensory decoder is learned along the paradigm in Figure 3, hypothesis H1 enables the learning operator $\approx$ to be replaced by an equality operator $=$, while hypotheses $\mathrm{H} 2$ and $\mathrm{H} 3$ enable the two terms on the right hand side of Equation (2) to be replaced by $P\left(M^{A g} \mid O_{S}^{A g}\right)$ and $P\left(S^{A g} \mid M^{A g}\right)$, respectively, which yields:

$$
P\left(O_{L}^{A g} \mid S^{A g}\right)=\sum_{M}\left(P\left(M^{A g} \mid O_{S}^{A g}\right) \cdot P\left(S^{A g} \mid M^{A g}\right)\right) .
$$

The right hand side of Equation (3) has now become the expression of the motor instantiation of the speech perception task, while the left hand side is the expression of the perception task instantiated within the framework of the auditory theory (see Figure 2). 
Therefore, if these three hypotheses are verified within a set of "perfect conditions" for learning, the sensory and motor models rely on the same information and make the same predictions. They are thus indistinguishable, whatever the test conditions might be.

When the indistinguishability theorem is satisfied, information encoded in the motor and sensory pathways is redundant. This shows that even when two theories or models are seemingly different - as the auditory and motor theories of speech perception appear to be they may be identical with respect to the computation they perform (as conceptualized by Marr, 1982, in his three-level framework, in which the same computational task can be carried out by algorithmic models with different representations; see also Laurent, Schwartz, Bessière, \& Diard, 2013).

Similar arguments are sometimes invoked in papers about auditory theories (e.g. Diehl et al, 2004, p. 168: "listeners do not recover gestures, but they do perceive the acoustic consequences of gestures. Any regularities of speech production (e.g., context dependencies) will be reflected in the acoustic signal and, through general mechanisms of perceptual learning, listeners come to make use of the acoustic correlates of these production regularities in judging the phonemic content of speech signals"). The indistinguishability theorem provides a theoretical basis based on Bayesian modeling to explain such more or less intuitive claims. More importantly, it suggests that what should drive our understanding of the respective roles of the auditory vs. motor systems in speech perception is related to what we are able to learn about them in the course of speech development. Understanding the potential role and complementarity of the sensory and motor recognition processes requires departing from the perfect conditions defined previously. Given the structure of the motor and sensory models, the possible differences between their predictions of perception tasks are strongly dependent on the information they encode, i.e., on how they were learned. 
The next parts of this article will introduce two sets of simulations providing two

461 directions in which auditory and motor theories depart from each other. Furthermore, some

462 fundamental sources of functional complementarity will be displayed.

Part 2 - The "auditory-narrow, motor-wide" framework for speech perception

In this part, we will focus on a generic property of COSMO that we consider largely independent from the specific implementation choices and that refers to structural aspects of property generates a natural complementarity between auditory and motor decoding processes, that we summarize by the so-called "auditory-narrow, motor-wide" framework. Finally we discuss the relationship between simulations and experimental data for speech perception development and speech processing in noise.

The sensory branch is narrow-band, the motor branch is wide-band: simulations within a simplified one-dimensional sensory-motor space structural difference between the two processes. While sensory perception implements a direct association between the sensory input $S$ and the perceived object $O_{L}$, motor perception appears to be more complex. Indeed the pathway from $S$ to $O_{S}$ involves motor information, $M$.

480 This suggests that motor recognition might require more time or cognitive resources before 481 convergence in the learning process, compared to sensory recognition. A possible 482 consequence is that the sensory system should be able to focus more rapidly and efficiently on 483 the set of exogenous learning stimuli provided by the environment, while the motor system 484 "wanders" through the sensory-motor space and endogenously explores regions, possibly 
different ones from the exogenous input. This would provide the sensory and motor systems with what we have called a "narrow-band" vs. "wide-band" specificity with respect to the learning data. The latter would be less efficient for learned stimuli, but would function better in adverse conditions involving unlearned stimuli.

This is what we set out to demonstrate, on a highly simplified theoretical framework based on 1-D motor and sensory variables linked by a sigmoid transformation. In this section the variables of the COSMO model are constrained to be very simple and are instantiated as follows: $M$ and $S$ are 1-D and discrete (with values regularly distributed between -15 and 15), while $O_{S}$ and $O_{L}$ both denote two possible objects $o_{1}$ and $o_{2}$. The Master Agent and the Learning Agent correspond to two different instances of the COSMO model with the same parametric forms (mostly Gaussian probability distributions) mathematically encoding the knowledge stored in the models. The two types of agent only differ by the values of the parameters of these parametric forms (for instance, means and standard deviations of the Gaussian probability distributions). We consider a supervised learning situation, where the parameters of the Master Agent and of the motor-to-sensory transformation performed by the simulated environment are fixed and the Learning Agent determines values for its parameters of internal representations through interactions with the Master according to the supervised learning scenario shown in Figure 3. We now describe the probability distribution forms and the parameters that are constant throughout learning. The prior objects $P\left(O_{S}\right)$ for both types of agent are set as uniform probability distributions; objects $o_{1}$ and $o_{2}$ are produced by the Master with the same frequency and the Learning Agent has no prior knowledge of the frequency of object apparition.

For both types of agent, motor repertoire probability distributions $P\left(M \mid O_{S}\right)$ are encoded as Gaussian probability distributions. For instance, to select a motor command corresponding to object $o_{1}$, the Master Agent draws a value of $M^{\text {Master }}$ according to the 
probability distribution $P\left(M^{\text {Master }} \mid\left[O_{S}^{\text {Master }}=o_{1}\right]\right)=\operatorname{Gauss}\left(\mu_{1}^{M}, \sigma_{1}^{M}\right)$, where the mean value $\mu_{1}^{M}$ of the Gaussian probability distribution corresponds to a prototypic motor gesture and the standard deviation $\sigma_{1}^{M}$ quantifies the variability of the Master Agent's production. In the Master Agent model, we set $\mu_{1}^{M}=-5, \mu_{2}^{M}=5$ and $\sigma_{1}^{M}=\sigma_{2}^{M}=1$ (see Figure 4, bottom plot).

The motor-to-sensory transformation $P(S \mid M)$ occurring in the environment is modeled as Gaussian probability distributions. More precisely, when the Master Agent issues a motor command $m$, the Learning Agent receives a value of the sensory input $S^{A g}$ drawn according to the probability distribution $P\left(S^{A g} \mid\left[M^{\text {Master }}=m\right]\right)=\operatorname{Gauss}\left(\mu_{m}^{S}, \sigma_{m}^{S}\right)$, where the value $\mu_{m}^{S}=f(m)$ is given by a function $f$ modeling the motor-to-sensory transformation and $\sigma_{m}^{S}=\sigma^{E n v}=1$ is a constant encoding the communication noise at learning time.

\section{***FIGURE 4 ABOUT HERE***}

Next, we consider nonlinear monotonous transformations, to keep some level of generality. Interestingly, nonlinear motor-to-sensory transformations have been exploited by the Quantal Theory of Speech (Stevens, 1972) as providing natural category boundaries for phonetic contrasts. In the Quantal Theory of Speech, it is proposed that such nonlinearities lead to the existence of articulatory plateaus, where variations in the articulation input lead essentially to no, or only small, acoustic variations. These are separated by discontinuity regions, where a small articulatory variation results in a strong acoustic jump. Stevens (1972, 1989) suggested that human languages exploit such discontinuities to set universal phonetic contrasts. This principle was confirmed in COSMO simulations of the emergence of phonological systems (Moulin-Frier et al., 2015). In the present study, we define the physical link $f$ between the motor gestures $M$ and their sensory consequences $S$ as a sigmoid function $f(m)=b \cdot \frac{\tan ^{-1}(a \cdot m)}{\tan ^{-1}(a \cdot b)}$, which is shown in Figure 4 (top left plot). Parameter $a$ allows the slope 
value of 12 , slightly lower than the $\mathrm{M}$ and $\mathrm{S}$ values of 15 and we set $a$ to either 0.01 in a quasi-linear case, or 0.1 to obtain a nonlinear case compatible with the Quantal Theory. The corresponding probability distributions $P\left(S^{A g} \mid\left[O_{S}^{\text {Master }}=o_{1}\right]\right)$ and $P\left(S^{A g} \mid\left[O_{S}^{\text {Master }}=\right.\right.$ $\mathrm{O}_{2}$ ]) are displayed in Figure 4 (top right plot). In the nonlinear case they are naturally more widely separated in sensory space than the equivalent distribution in motor space. At this stage, we consider that the Master, along with the Learning Agent, have the same nonlinear transformation between their motor and sensory variables. A major departure from this assumption would concern differences in vocal tract shape mainly associated with age and sex. We consider that all agents are equipped with a normalization mechanism enabling them to transform sensory information provided by the Master into an $\mathrm{S}$ value appropriately situated in their internal sensory space. Such normalization processes exist and have been displayed since the first months of age (e.g. Kuhl, 1979, 1983; Polka, Masapollo and Ménard, 2014). Once the stimuli are transmitted by the Master to the Learning Agent and have been appropriately normalized, the nonlinear transformation is of no further use to the Master Agent. Hence, remaining differences between such transformations in the case of the Master and the Learning Agent play no role in further processing in COSMO. We will return in the discussion of Part 2 to consider how realistic normalization processes could modify the present simulations.

In the computer simulations presented below, all Gaussian probability distributions are truncated: we define a baseline value $\varepsilon=10^{-5}$ and probability values below this threshold are set to $\varepsilon$; the probability distribution is normalized afterward ${ }^{(3)}$. This avoids cognitively implausible numerical precision of probability distributions, which would yield unwanted side effects. For instance, in classification tasks, when comparing the predictions of Gaussian models too far from their mean values, an infinitely precise model generalizes too well and behaves like an analytical model, with a precise classification frontier and abruptly changing 
560

561

562

563

564

565

566

567

568

569

570

571

572

573

574

575

576

577

578

579

580

581

582

583

584

responses. In the present simulations, probability distributions, truncated in this manner, degenerate outside of their "competence domains" and classification responses behave according to chance when exotic stimuli are presented.

\section{Learning in COSMO}

Hypotheses $\mathrm{H} 1, \mathrm{H} 2$ and $\mathrm{H} 3$ are at the basis of the indistinguishability theorem, expressing unrealistic perfect learning conditions. We now add a plausible learning algorithm to the hypotheses and we will describe how the result departs from ideal learning conditions and ultimately enables sensory and motor recognition processes to be distinguished.

Learning follows the interaction paradigm introduced as Figure 3. To recapitulate the learning scenario, both the Master and Learning Agent interact in a simulated environment. Probability distributions defining the Master Agent and motor-to-sensory transformation of the environment are set constant during learning. The Master Agent provides the Learning Agent with $<$ object, stimulus $>$ pairs (later referred to as $\langle o, s\rangle$ ). The Learning Agent ascertains from this data both its sensory and motor classification systems; more precisely, it identifies parameters for its sensory prototypes $P\left(S^{A g} \mid O_{L}^{A g}\right)$, internal model $P\left(S^{A g} \mid M^{A g}\right)$ and motor repertoire $P\left(M^{A g} \mid O_{S}^{A g}\right)$, which are all implemented using Gaussian probability distributions. To express the fact that the Learning Agent starts without any knowledge, initial states of all these Gaussian probability distributions are characterized by values of mean parameters $\mu$ at the center of their domains and initial standard deviation values $\sigma$ that are large relative to the domain size. This approximates to uniform probability distributions.

To allow fair comparisons of the sensory and motor instantiations of the speech perception task, the components of the sensory and motor classification systems are learned independently and using the same data. 
In the sensory recognition system based on a direct association between stimuli and

objects, firstly we learn sensory prototypes of the form $P\left(S^{A g} \mid\left[O_{L}^{A g}=o\right]\right)=\operatorname{Gauss}\left(\mu_{o}^{S}, \sigma_{o}^{S}\right)$ which correspond to each object. Learning consists of computing a Gaussian probability fit distribution $P\left(S^{A g} \mid O_{L}^{A g}\right)$ from $<o, s>$ pairs. Each time the Learning Agent receives such a pair from its Master, the values of the mean $\mu_{o}^{S}$ and of the variance $\sigma_{o}^{S}$ of the corresponding Gaussian probability distribution are updated accordingly. An extensive study of the dynamics of learning sensory prototypes and their effects on the resulting classifiers is provided by Kleinschmidt \& Jaeger (2015); as such dynamics are not the focus of our contribution, we implement a straightforward learning procedure, where the order of learning data has no effect.

The motor recognition system exploits the same $\langle o, s\rangle$ pairs to learn both components of its pathway, namely the internal model of the motor-to-sensory transformation $P\left(S^{A g} \mid M^{A g}\right)$ and the motor repertoire $P\left(M^{A g} \mid O_{S}^{A g}\right)$. We exploit a Learning by Accommodation algorithm, which allows learning of the two components at the same time. Importantly, this algorithm takes into account "babbling drift", i.e. the fact, presented in the Introduction, that the agent should not explore systematically and uniformly its sensory-motor space but rather should focus on regions of interest provided by the Master's sounds. The accommodation algorithm enables the Learning Agent to progressively focus on the Master's stimuli, making learning quicker and more efficient (Barnaud, Schwartz, Diard, \& Bessière, 2016).

The algorithm involves a simple imitation paradigm without any error measurements. It works in the following way:

(i) The Learning Agent tries to mimic the sensory input $s$ of the $\langle o, s\rangle$ pair provided by the Master, by producing a motor command $m$ given its current state of knowledge, the input stimulus $S$ and the input object $o$. After probabilistic inference, this amounts to 
randomly drawing a value for $m$ according to the following probability distribution:

$$
P\left(M^{A g} \mid\left[S^{A g}=s\right]\left[O_{S}^{A g}=o\right]\right)=P\left(M^{A g} \mid\left[O_{S}^{A g}=o\right]\right) . P\left(\left[S^{A g}=s\right] \mid M^{A g}\right) .
$$

Equation (4) shows that the choice of motor commands $m$ is driven by two factors:

first, the need to match the stimulus $s$ given by the Master, as predicted by the current state of knowledge encoded in the internal model $P\left(S^{A g} \mid M^{A g}\right)$; second, the tendency to use the same motor commands that were previously associated with the object $o$ communicated by the Master, as stored in the motor repertoire $P\left(M^{A g} \mid O_{S}^{A g}\right)$.

(ii) Once selected, the motor command $m$ is performed and has a sensory consequence $s^{\prime}$. The Learning Agent then uses the observed correspondence of $s^{\prime}$ and $m$ to improve the internal model by updating the parameters $\mu_{m}^{S}$ and $\sigma_{m}^{S}$ of the probability distribution $P\left(S^{A g} \mid\left[M^{A g}=m\right]\right)$. It also exploits the selected value $m$ in its motor repertoire by updating the $\mu_{o}^{M}$ and $\sigma_{o}^{M}$ parameters of the probability distribution $P\left(M^{A g} \mid\left[O_{S}^{A g}=o\right]\right)$. Therefore, the algorithm progressively refines the internal model of motor-to-sensory mapping, both with some endogenous random exploration due to inaccurate imitation in the first stages of the learning process and with a progressive focus on the learning stimuli that result in a better mapping around the regions of the stimuli provided by the Master. In parallel, the algorithm progressively anchors adequate motor gestures for each object, i.e. gestures producing sounds that correspond to the sensory distribution produced by the Master for the object.

\section{Simulation results}

\section{Learning pace: fast and focused sensory learning vs. slow and diffuse motor}

\section{learning.}

\section{***FIGURE 5 ABOUT HERE***}

We use the evolution of entropy $H(P(X))=-\sum_{i} P\left(X=x_{i}\right) \log P\left(X=x_{i}\right)$ as a 
numeric indicator that quantifies how much information becomes stored in the probability distributions of the models. We compare learning speeds using the evolution of $H\left(P\left(S^{A g} \mid O_{L}^{A g}\right)\right)$, the entropy of the sensory model on the one hand and $H\left(P\left(S^{A g} \mid O_{S}^{A g}\right)\right)$, the entropy of the motor model on the other hand. $H\left(P\left(S^{A g} \mid O_{S}^{\text {Master }}\right)\right)$, the entropy of the probability distribution over the stimuli produced by the Master Agent, which is constant during learning, is used as a reference. Each of these entropy values is actually a set of measurements, one for each possible object value. We therefore average them over objects and, since we have two objects in these 1-D experiments, consider $\frac{1}{2} \sum_{O_{L}^{A g}} H\left(P\left(S^{A g} \mid O_{L}^{A g}\right)\right)$ for the sensory model, $\frac{1}{2} \sum_{O_{S}^{A g}} H\left(P\left(S^{A g} \mid O_{S}^{A g}\right)\right)$ for the motor model and $\frac{1}{2} \sum_{O_{S}^{M a s t e r}} H\left(P\left(S^{A g} \mid O_{S}^{\text {Master }}\right)\right)$ as the Master's reference.

The corresponding curves are displayed in Figure 5 for the two values of nonlinearity in the motor-to-sensory transformation. This Figure shows that the entropy of the sensory model converges quickly to a level close to the entropy of the stimuli produced by the Master, while the entropy of the motor model converges more slowly. In the linear case, the sensory model is able to converge to exactly the same entropy as that of the Master Agent, whereas it remains larger in the nonlinear case because the constraint that distributions $P\left(S^{A g} \mid O_{L}^{A g}\right)$ are Gaussian leads to some residual discrepancy between the models of the Master Agent and Learning Agent. However, whatever the nonlinearity, the motor model entropy $H\left(P\left(S^{A g} \mid O_{S}^{A g}\right)\right)$ decreases more slowly than the sensory model entropy, indicating slower learning. This corresponds to our prediction that the inference process is more complex in the motor model. Hence the learning mechanism is slower and less efficient, since it "visits" portions of the sensory-motor space that are not available to the sensory recognition system. 


\section{***FIGURE 6 ABOUT HERE***}

To support this point, let us recall that learning the motor $P\left(S^{A g} \mid O_{S}^{A g}\right)$ model consists of learning both $P\left(S^{A g} \mid M^{A g}\right)$ and $P\left(M^{A g} \mid O_{S}^{A g}\right)$. We show in Figure 6 an instance of an internal $P\left(S^{A g} \mid M^{A g}\right)$ model in the nonlinear case learned by the agent after 20,000 iterations of learning by the accommodation algorithm. Data presented in Figure 6 first show that the shape of the motor-to-sensory transformation has been adequately learned. However, it appears that some regions are learned better than others: these regions, where the variance of the $P\left(S^{A g} \mid\left[M^{A g}=m\right]\right)$ distribution is small, correspond to those of the sensory space where stimuli have been provided by the Master Agent. Other regions, far from the data of the learning set, have a higher variance. However, the Learning Agent has acquired global knowledge, which provides the motor learning process with what we could call a more "diffuse" character.

In this learning process, we have implemented a mechanism that naturally leads to the departure from both hypotheses H1 and H2 of the "perfect learning conditions". Since learning is intrinsically incomplete, the Learning Agent cannot fully internalize all the production abilities of the Master Agent. This results in complementarity between the sensory and motor models. While the sensory system can focus on the stimuli provided by the Master Agent and learn them quickly and efficiently, the motor system has to learn both a sensorymotor model and a motor repertoire. This more complex process is slower and less focused on the learning set, because it requires exploring an intermediate motor space. However, this can be useful for unlearned conditions as we will assess next.

\section{motor model is more robust in noisy conditions.}

In this section we compare the models' robustness to communication noise in an 
683

684

685

evaluation experiment where the Learning Agent interacts with a Master Agent defined in the same way as previously, except that after the learning phase, we introduce a test phase where we vary the standard deviation $\sigma_{m}^{S}=\sigma^{E n v}$ of the Gaussian probability distribution $P\left(S^{A g} \mid\left[M^{\text {Master }}=m\right]\right)$, thus encoding various levels of environmental noise.

The Master Agent provides $<o, s>$ pairs of a given noise level and the agent estimates the object $o^{\prime}$ from the stimulus $s$ using either sensory recognition, i.e. by computing the probability distribution $P\left(O_{L}^{A g} \mid S^{A g}\right)$, or motor recognition, i.e. by computing $P\left(O_{S}^{A g} \mid S^{A g}\right)$, as defined in Figure 2. Sensory recognition is implemented as a Gaussian classifier obtained by probabilistic inversion of the sensory prototypes $P\left(S^{A g} \mid O_{L}^{A g}\right)$ :

$$
P\left(O_{L}^{A g} \mid S^{A g}\right)=\frac{P\left(S^{A g} \mid o_{L}^{A g}\right)}{\sum_{O_{L}^{A g}} P\left(S^{A g} \mid o_{L}^{A g}\right)}
$$

Motor recognition is implemented according to:

$$
P\left(O_{S}^{A g} \mid S^{A g}\right) \propto \sum_{M^{A g}}\left(P\left(M^{A g} \mid O_{S}^{A g}\right) P\left(S^{A g} \mid M^{A g}\right)\right) .
$$

Comparing the values of the object intended by the Master Agent, $o$, and that estimated by the Learning Agent, $o^{\prime}$, we compute confusion matrices and define the recognition rate as the mean of their diagonal coefficients.

***FIGURE 7 ABOUT HERE***

In Figure 7, we present the mean values of recognition rates for the linear and nonlinear cases. The scores are provided at three learning stages (after learning 500, 2,000 or $20,000<o, s>$ pairs), for a range of noise degradation, from no added noise to stimuli corrupted by high levels of noise (noise is indexed by variation of the $\sigma^{E n v}$ value). First, we observe a large effect of nonlinearity on the sensory classifier, with a sharp decline of performance with noise in the nonlinear case. This derives from more pointed and separated probability distributions (Figure 4, top right panel). The observations that follow are independent of nonlinearity. 
Second, since the sensory system learns rapidly, it has already converged before 500

learning iterations and does not evolve afterwards. It provides good recognition scores without noise, with a quick degradation of performance when noise is added.

Third, in contrast the motor system appears to learn slowly. At the beginning of the learning process (top row in Figure 7), it performs very poorly and the decrease of the recognition rate as noise increases is slower than for the sensory model. When learning proceeds with more iterations, the motor system performs increasingly well, the general trend being that it becomes better than the sensory model in noisy conditions, though still remaining poorer in the absence of noise. At the last stage of the learning process $(20,000$ iterations) the two models give rather similar performances (we tend towards the "perfect learning conditions" of the indistinguishability theorem).

Fourth, and finally, the perceptuo-motor model implementing a Bayesian fusion of the sensory and motor recognition models according to the Equation shown in Figure 2 performs better than the two isolated models under all conditions.

\section{***FIGURE 8 ABOUT HERE***}

We now explore how the sensory system is more efficient in the absence of noise and how the motor system is more efficient in its presence. This is illustrated in Figure 8, where we display probability distributions for the two objects, for both motor and sensory systems. Furthermore, we show the example $s_{\text {clean }}$ stimuli for a stimulus under normal conditions, i.e. without added noise, and $s_{\text {noise }}$ for a stimulus in adverse conditions, i.e. with added noise. When the "typical" $s_{\text {clean }}$ stimulus is considered, it is close to prototypes of the motor and sensory models, i.e. to the modes of corresponding probability distributions. However, the sensory model, being of lower variance than the motor model, yields a less uncertain probability distribution categorization than the motor process. The two models correctly recognize object $o_{2}$ as the cause of the $S_{\text {clean }}$ stimulus, but the sensory model is slightly more 
certain of perception than the motor model is.

When the "noisy" stimulus ( $s_{\text {noise }}$ ) is considered, it is far from prototypes of both motor and sensory models. However, the motor model, being of greater variance than the sensory model, generalizes better. Whereas for the sensory model, probability distributions quickly fall below the $\varepsilon$ threshold we defined, yielding random categorization, the motor model is more robust and conserves categorization capabilities.

\section{Concluding Part 2: summary and predictions}

The present simulations let a major difference between auditory and motor learning appear. Auditory learning is rapid and, by definition, perfectly focused on the acoustic stimuli provided by the Master. In fact, the auditory system in COSMO is an "ideal processor" of acoustic input, as in many previous Bayesian models (e.g. Norris \& McQueen, 2008;

Kleinschmidt \& Jaeger, 2015). However, the intrinsic limitation is provided by departures from exactly what has been learned. This is where the motor system may become relevant. Indeed, the motor system is intrinsically slower since it has a more complex inference process to deal with. It is also less well tuned to the learning corpus, because of the existence of an intermediate motor representation in the inference process. But it is this more complex learning process that supplies the possibility of wandering around stimuli and configurations that are not contained in the learning set provided by the environment. This is what makes it "wider" and hence better able to process unknown stimuli.

It is important to stress at this stage that the auditory-narrow, motor-wide hypothesis appears to be generic, i.e. intimately related to the basic COSMO structure, because of the more complex structure of the motor inference process compared with the auditory one (see Figure 2). We had to propose a number of technical and non-generic choices to perform simulations in this part of the article. These include: (i) the motor-to-sensory transformation 
was presumed to be nonlinear but monotonous, (ii) the Master was considered to be physically similar to the Learning Agents, with the same nonlinear motor-to-sensory transformation, supposing that the normalization process was solved in some way, (iii) only one Master was introduced into the learning process, while an infant typically has to deal with a number of Masters to learn from in the environment.

More realistic simulations, involving: non-monotonous motor-to-sensory transformations, variations of transformations from one agent to the other, possibly in relation with normalization processes between agents with different sizes and shapes of their vocal tract, multiple Master Agents in the learning process, would basically result in a large increase in complexity of the sensory-to-motor inference process and hence in an increase toward the trend for slow and diffuse motor learning. In some sense, the 1-D simulations presented in Part 2 minimize the trend towards the auditory-narrow vs. motor-wide contrast, which is likely to be larger in a more realistic simulation with COSMO - as will be displayed in Part 3. Therefore, it can safely be claimed that the auditory-narrow motor-wide hypothesis is an intrinsic property of the COSMO structure, and probably an intrinsic characteristic of motor vs. auditory decoding in a perceptuo-motor theory of speech perception.

This property of the model generates two predictions, in the sense that two consequences follow directly from the property. These consequences were not considered during modeling; they are logically entailed by the model. These predictions are in line with already available data and observations pertaining to speech development and processing.

\section{Prediction 1 - auditory learning should be more rapid than motor learning}

A strong prediction in COSMO is that auditory learning, which typically consists of learning the sensory distributions $\mathrm{P}\left(\mathrm{S}^{\mathrm{Ag}} / \mathrm{O}_{\mathrm{L}}{ }^{\mathrm{Ag}}\right)$, is a simpler process than motor learning i.e. learning the motor distributions $\mathrm{P}\left(\mathrm{M}^{\mathrm{Ag}} / \mathrm{O}_{\mathrm{S}}{ }^{\mathrm{Ag}}\right)$. It is well-known that the auditory system is developmentally mature before the motor one, as is reviewed in the Introduction, but this is 
782

783

784

785

786

787

788

789

790

791

792

793

794

795

796

797

798

799

800

801

802

803

804

805

806

generally only related to biological constraints. Firstly, audition begins to mature before birth, as is displayed by the sensitivity of newborns to language (Mehler, Jusczyk, Lambertz, Halsted, Bertoncini, \& Amiel-Tison, 1988) or to the voice of their mother (DeCasper \& Fifer, 1980). Secondly, critical periods seem to shape the course of development of speech perception and production towards the mature stage (see a recent review in Werker \& Hensch, 2015). Importantly, the present simulations suggest that an additional factor could be provided by the complexity of the learning process. In this respect, it is of interest to mention that even for vowels, language tuning in production has never been described before 10 months of age (e.g. de Boysson-Bardies et al., 1989) while it occurs in speech perception as soon as 6 months of age (Kuhl et al., 1992), though infants are capable of producing vowel-like vocalizations almost since birth and display vocal imitations as early as 4 months of age (Kuhl \& Meltzoff, 1996).

It would be of great interest in this discussion to attempt to correlate observed delays in the developmental schedule with some measurement of differences in the learning period or entropy reduction in a Figure such as Figure 5. However, this seems far from any reasonable prediction at the present state of possible simulations.

\section{Prediction 2 - motor processing should be more important in adverse conditions}

This is the major prediction of the auditory-narrow motor-wide hypothesis. Indeed, it is proposed as an intrinsic COSMO property that the motor system should be less efficient than the auditory system in learned conditions, while the motor system gains efficiency in unlearned ones, e.g. in noisy or adverse conditions. A likely consequence of this prediction is that the motor system should be more involved in such adverse conditions. As reported previously, this is exactly what is regularly observed for neurocognitive data, with an increased BOLD (Blood-Oxygen Level Dependent) activity in fMRI (functional Magnetic Resonance Imaging) data in motor regions for noisy (Binder et al., 2004; Zekveld et al., 
807 2006), or non-native stimuli (Callan et al., 2004, 2014; Wilson \& Iacoboni, 2006). This is also

808 in line with evidence for motor perturbations seen in auditory perception only in noisy

809 conditions (e.g. d'Ausilio et al., 2012 vs. 2009), or for ambiguous stimuli around a phonetic

810 boundary (e.g. Möttönen \& Watkins, 2009; Rogers et al., 2014).

811

812 


\section{Part 3 - Extracting perceptuo-motor invariance in syllabic units}

While in the previous part the focus was on generic properties of the COSMO model, we now move towards non-generic properties associated with the specific way auditory and motor information is most probably distributed in a specific case of phonetic sequences made of CV syllables with a C stop consonant and a V oral vowel. This part of the article will deal with a problem that has long been considered crucial in the debate about auditory vs. motor theories, namely the invariance problem. The question of invariance has often been raised by motor theorists around the alleged lack of acoustic invariance for the plosive place of articulation, considering that in this specific case motor invariance was straightforward (see below). However, the case of vowels seems different and it has already been suggested that invariance could be of a different nature for vowel vs. plosive place of articulation, which would be auditory in one case and articulatory in the other (see Bailly, 1997; Kröger et al., 2009, 2014). Therefore, COSMO appears as a perfect framework for dealing with this question in a perceptuo-motor framework.

To address the question of auditory vs. motor invariance for vowel vs. plosive place of articulation, we will need to introduce specific knowledge and hypotheses concerning CV syllable production, perception and development. Furthermore, simulations will be carried out on a specific model of the vocal tract, VLAM (variable linear articulatory model), enabling generation of articulatory and acoustic configurations associated with CV sequences. Finally, simulations will include specific simplifications about sensory and motor variables, as well as about the learning process.

In light of this specific implementation of COSMO for syllables, which we will call COSMO-S, the question we address is: in the distribution of information for plosives and vowels, is there any potential evidence for differentiation of the motor and auditory systems in extracting phonetic invariance from acoustic stimuli? We will firstly present a literature 
review on the perception and production of $\mathrm{CV}$ syllables in relation to the place of articulation cues. Then we will describe the vocal tract model VLAM, and the COSMO-S version of COSMO for syllable perception and production, together with the way learning is implemented in COSMO-S. Finally, we will describe simulations with COSMO-S and explore what light they might shed on the question of vowel vs. plosive place of articulation invariance.

\section{Auditory or motor cues for vowel vs. plosive place of articulation}

The question of the plosive place of articulation invariance has long been considered as a crucial test for auditory vs. motor theories of segmental invariance. On the one hand, partisans of motor theories have regularly mentioned it as a typical case, where auditory invariance was out of reach while motor invariance would be directly available (Liberman et al., 1967; Liberman \& Mattingly, 1985). On the other hand, the classical objection to the motor theory is the probable complexity of the cognitive or computational implementation of the inversion process that would enable the listener to recover the proposed motor invariant from the acoustic speech input: the labial gesture for bilabials, the tongue apex gesture for coronals, the tongue dorsum gesture for palato-velars.

Partisans of auditory theories have also searched possible invariant auditory cues that characterize the plosive place of articulation. The pioneer work by Delattre, Liberman, \& Cooper (1955) on the "acoustic locus" actually served as a precursor for both auditory and motor proposals on invariance. In the framework of his Quantal Theory, Stevens proposed at the end of the 1970s that there might be a local spectral invariant for the plosive place of articulation, located around the position of the acoustic burst and independent of the speaker, the plosive manner of articulation and the context. Bilabial spectra would be "diffuse falling" (with energy all over the spectrum but more of it at low frequencies), alveolars would be 
863

864

865

866

867

868

869

870

871

872

873

875

876

877

878

879

880

881

882

883

884

885

886

887

"diffuse raising" (idem but with more energy at high frequencies) and velars would be compact (with most of the energy packed into the medium) (Blumstein \& Stevens, 1979; Stevens, 1980; Stevens \& Blumstein, 1978). Following further proposals by Kewley-Port (1983), a progressive shift was made towards dynamic cues associating spectral values with the plosive and the next vowel. At the end of this process, the locus came back with the "locus equations" introduced by Sussman (Sussman, Fruchter, Hilbert, \& Sirosh, 1998; Sussman, Hoemeke, \& Ahmed, 1993; Sussman, McCaffrey, \& Matthews, 1991) assuming relational invariance (correlations between F2 values for the plosive and the next vowel) as a correlate of the place of articulation. Importantly, acoustic characterization of the plosive place of articulation seems basically to rely on spectral data at two instants; plosive release and vowel climax.

Our proposal is different. In the PACT framework and in light of the perceptuo-motor developmental schedule described at the beginning of this paper, we presume that in a first stage, speech perception would benefit from rapidly maturing auditory processing that enables infants to categorize all CV sequences available in their environments. In this first stage, the motor system would not be mature and probably not even completely functionally related to the speech perception system according to Kuhl et al. (2014). Hence, the infants would not have at their disposal invariant cues for the plosive place of articulation. This question is debated, with negative results on plosive invariance before 6 months in Bertoncini, BijeljacBabic, Jusczyk, Kennedy, \& Mehler, 1988; Eimas, 1999; vs. data suggesting the possibility to discriminate /b/ from /d/ at 6 months of age, Hochmann \& Papeo, 2014; and a discussion on possible confounding effects in Dole, Loevenbruck, Pascalis, Schwartz, \& Vilain, 2015. In a second stage, after 7 months there is progressive coupling and maturation of the speech motor system. Then, infants could discover that plosive-vowel sequences heard in the environment are produced by specific movements of the lips for bilabials, and the tongue apex 
or dorsum for alveolars or velars. Hence, the content of the motor repertoire would enhance perceptual representations and allow invariance to emerge in a perceptuo-motor space. For vowels, the situation is probably different. Indeed, auditory representations for oral vowels have been described in a number of studies, and oral vowels seem properly characterized in all their phonetic dimensions in a bundle of frequency parameters (e.g. (F1F0) for height, (F2-F1) for place of articulation and $F^{\prime} 2$ for rounding; all values are in Barks: see Ménard, Schwartz, Boë, Kandel, \& Vallée (2002). In contrast, the articulatory characterization of oral vowels is less straightforward (e.g., Boë, Perrier, \& Bailly, 1992) and perturbation experiments suggest that invariants for vowels could be auditory rather than motor (Savariaux, Perrier, \& Orliaguet, 1995; Savariaux, Perrier, Orliaguet, \& Schwartz, 1999). It is more in terms of vowel reduction that articulatory dynamics could play a role, though the debate on this topic was vigorous in the 1980s and 1990s (e.g., Strange (1989) vs. Nearey (1989) or Perrier, Lœvenbruck, \& Payan (1996) vs. Pitermann (2000)). Therefore, our hypothesis is that the auditory and motor systems could be complementary in terms of the content of their representations for phonetic invariance, motor or gestural cues probably being crucial for the plosive place of articulation, while auditory parameters would be efficient for vowel characterization ${ }^{(4)}$. This is what we now propose to test with COSMO. For this aim, since natural articulatory data are sparse, particularly about perceptuo-motor development early in life, we will use synthetic data in the framework of the articulatory model of the vocal tract, VLAM.

\section{VLAM and the generation of synthetic CV syllables}

VLAM is a realist vocal tract model (Maeda, 1990) thanks to which seven articulatory parameters (Jaw, Larynx, TongueBody, TongueDorsum, TongueApex, LipHeight, LipProtrusion) have been derived from a guided principal component analysis of 
913 cineradiographic images of the vocal tract. These allow the description of the jaw and larynx

914 position, and of the tongue and lips shape. The parameters can be interpreted in terms of

915 phonetic and muscular commands (Maeda \& Honda, 1994). The areas of 28 sections of the

916 vocal tract are estimated as linear combinations of these seven parameters, which then allows

917 computation of the transfer function and formants (Badin \& Fant, 1984) (see Figure 9).

$918 \quad * * *$ FIGURE 9 ABOUT HERE***

919 In short, VLAM is a geometric model enabling formants from articulatory parameters

920 to be computed. This model has been evaluated over the last fifteen years in terms of its

921 ability to generate vowels and plosive stimuli compatible with data from infants (Boë et al.,

922 2013), children and adults (Laurent et al., 2013; Ménard et al., 2002; Ménard, Schwartz, \&

923 Aubin, 2008; Ménard, Schwartz, \& Boë, 2004; Schwartz, Boë, Badin, \& Sawallis, 2012b). It

924 is also the articulatory synthesizer of the DIVA (Directions Into Velocities of Articulators)

925 model of speech production (Guenther, 2006; Guenther, Hampson, \& Johnson, 1998).

926 Here, VLAM is considered as a simplified implementation of the motor-to-auditory

927 relationship in the human vocal tract ${ }^{(5)}$. It is used both to generate CV syllables thought to be

928 produced by the Master Agent and as an external simulator of the Learning Agent' vocal tract

929 so that it can learn from the perceptual consequences of the motor commands it is sending.

930 VLAM also incorporates a model for vocal tract scaling associated with age, thanks to which

931 the size increases with age in a nonlinear way compatible with experimental data (see Boë et

932 al., 2013; Ménard et al., 2002, 2008). However, as in Part 2, we do not consider here vocal

933 tract differences between the Learning Agent and the Master, supposing that if there were any,

934 they could be solved by appropriate normalization processes (see Ménard et al., 2002).

$935 \quad$ Generation of oral vowels

936 Vowels are defined as articulatory configurations that are not too closed, so as not to 937 generate noise in their acoustic output. This is characterized in VLAM by setting a constraint 
on the constriction, which is the position of the section of the vocal tract with the smallest area. The constriction area for vowels is higher than a minimum value of $0.15 \mathrm{~cm}^{2}$. In the present set of simulations, we only considered the three extreme oral vowels $/ \mathrm{i}, \mathrm{a}, \mathrm{u} / \mathrm{,}$, which provide the preferred choice in human languages (see Schwartz, Boë, Vallée \& Abry, 1997). Any speech sound should need all 7 VLAM parameters for its complete generation and characterization. However, we have attempted to keep the number of free parameters at the smallest possible value to minimize later computations. Hence, vowels are described here by three VLAM articulatory parameters (TongueBody, TongueDorsum and LipHeight), all other parameters being set to a neutral value (resting position). We define motor vowel prototypes for /a i u/, using average formant values for French vowels (Meunier, 2007) as targets and selecting values of the three $V L A M$ parameters that best fit the acoustic target. For each category of vowel, we generated a set of articulatory configurations according to a Gaussian probability distribution centered on the prototype value.

\section{Generation of stop consonants}

Plosives are defined as articulatory configurations achieved just after a complete closure of the vocal tract, i.e. at the time of acoustic release, which typically generates an acoustic burst. In the present simulations we characterize plosives by the formants produced with a constriction close to, but still slightly higher than, zero, so as to be able to compute formants. We only considered the three extreme plosive places of articulation (labial, alveolar, velar) that provide the preferred choice in human languages (see Schwartz et al., 2012b). The unvoiced stop consonants $/ \mathrm{p}, \mathrm{t}, \mathrm{k} /$ corresponding to these places of articulation are more frequent in human language than their voiced counterparts $/ \mathrm{b}, \mathrm{d}, \mathrm{g} /$, but, in the rest of this paper, we keep the voiced set of consonants /b, d, g/, because voiced plosives provide the clearest formant trajectories and enable a better specification of formants at the beginning of the opening trajectory from the plosive to the next vowel. 
We adopt the view proposed by Öhman (1966) that plosives are local perturbations

964 (vocal tract closing gestures) of vowel configurations within CV syllables. Therefore, we synthesize plosives by closing the vocal tract from a vowel position, using the VLAM Jaw parameter combined with one other articulator, i.e. Jaw and LipHeight for /b/, Jaw and TongueApex for /d/, and Jaw and TongueDorsum for/g/. Hence, plosives are described by 968 five parameters (Jaw, TongueBody, TongueDorsum, TongueApex and LipHeight).

969 Furthermore, the perturbation gesture allowing a consonant to be produced from a vowel is 970 characterized by two parameters: the variation (Delta) of Jaw and another one from among LipHeight, TongueApex or TongueDorsum. To obtain a consonant, both articulators should be combined, so that the vocal tract constriction area reaches a value between 0.05 and $0.15 \mathrm{~cm}^{2}$.

973 More specifically, the set of consonants that can be achieved from a vowel configuration of 974 the vocal tract is the set of configurations obtained by 1) going through all possible discrete 975 values of the parameter Jaw and 2) for each of these values selecting the value of the other 976 articulator (LipHeight, TongueApex or TongueDorsum) such that when the perturbation is 977 applied to the vowel the constriction area is the closest possible to $0.05 \mathrm{~cm}^{2}$. The choice of 978 modeling a consonant as a perturbation added to a vowel means that consonants and vowels 979 are linked by maximal co-articulation.

\section{Representation of $\mathrm{CV}$ sequences} acoustic parameters, the question is to define an adequate representation of the trajectory from

$983 \mathrm{C}$ to V, characterizing the syllable in articulatory and acoustic terms. Since we showed in the 984 previous section that the data converge towards a characterization based on plosive onset and 985 vowel formants, plosive-vowel syllables are characterized as a pair of two articulatory states: 986 one for the plosive and the other for the vowel, neglecting the geometry and temporal aspects 987 of the trajectory linking these two states. Altogether, a CV sequence is associated in VLAM 
with 8 articulatory parameters, 5 for the plosive and 3 for the vowel.

In the acoustic space, vowels are characterized by their first two formants $(F 1, F 2)$,which $V L A M$ computes from the articulatory parameters in the open state. For plosives, where $F 1$ is basically the same for all configurations (around $250 \mathrm{~Hz}$ ), characterization is by $F 2$ and $F 3$, computed by $V L A M$ in the closed state. A CV sequence is associated to 4 acoustic parameters, 2 for the plosive and 2 for the vowel.

\section{***FIGURE 10 ABOUT HERE***}

Figure 10 displays the acoustic properties of the vowels and plosives generated. The representation of vowels in the $(F 1, F 2)$ plane is classical, with /i, a, $\mathrm{u} /$ at the corners of the vowel triangle (Figure 10, top). The representation of plosives in the $(F 2, F 3)$ plane is less common (Figure 10, bottom). It has been extensively discussed in Schwartz et al. (2012b). We observe that there is a trend toward lower $(F 2, F 3)$ values for $/ \mathrm{b} /$, higher values for $/ \mathrm{d} / \mathrm{and}$ medium values for /g/. This recalls the "diffuse falling" vs. "diffuse raising" vs. "compact" contrasts proposed by Stevens and Blumstein (1978), but with considerable variations of the plosive recognition depending on the vowel context.

\section{***FIGURE 11 ABOUT HERE***}

We show in Figure 11 the relationship between the $F 2$ values for plosives and vowels, providing a portrait that is globally coherent with the one reported by Sussman et al. (1998) for natural speech.

The two-state implementation of syllabic trajectories is highly simplified in relation to natural CV sequences, and a number of more elaborate CV co-articulation models have been suggested since the pioneer one proposed by Öhman (1966). However, here we merely aimed to generate syllables whose variability patterns were similar to the complexity of real speech signals. The syllable material displayed in Figure 10 provides an adequate compromise. It corresponds to complex variations in an 8-D articulatory space and resulting in variations in a 
1013 4-D acoustic space with co-articulation patterns that are globally coherent with those of

1014 natural syllables. We will next examine how the motor and auditory systems of the COSMO

1015 model extended to syllables can deal with this variability.

1016

\section{COSMO-S, an extension of the COSMO model to process plosive-vowel syllables}

We have extended the COSMO model to $\mathrm{CV}$ syllable processing. The objects, $O_{S}$ from the speaker's point of view, and $O_{L}$ from a listener's perspective, refer to the syllables we consider: /ba/, /bi/, /bu/, /ga/, /gi/, /gu/,/da/, /di/,/du/. Since we model a syllable as a vowel state and a consonant state, the variable $S$ separates into $S_{V}$ and $S_{C}$, and the variable $M$ into $M_{V}$ and $M_{C}$. Apart from that, the COSMO-S model (see Figure 12, top) shares its global structure with COSMO as it is made of the same systems: (i) the auditory system associates sensory representations with the corresponding $O_{L}$ syllable labels; (ii) the sensory-motor system associates motor and sensory representations; (iii) the motor system associates motor representations with $O_{S}$ syllable labels.

These systems are linked by $\lambda$ coherence variables, which are a mathematical tool

1029 used to force duplicate variables to have the same values at all times during probabilistic

1030 inference (Bessière et al., 2013; Gilet, Diard, \& Bessière, 2011). This provides a mathematical

1031 implementation of a probabilistic switch, allowing the different parts of the model to be

1032 activated or deactivated during probabilistic inference, thus permitting constraints coming

1033 from the different sub-models to be integrated into the global model. Likewise, the

1034 specification of $C=$ True in an inference task allows the combination of motor and auditory 1035 cues.

1036 The auditory system describes the knowledge the agent has of the link between $O_{L}$ 1037 syllables and sensory variables: $S_{V}^{\prime}\left(F 1\right.$ and $F 2$ for the vowel) and $S_{C}^{\prime}(F 2$ and $F 3$ for the 
1038

1039

consonant). These are implemented as 4-D Gaussian probability distributions, the mean vectors and covariance matrices of which are estimated during the learning process (see below).

The sensory-motor system describes the knowledge the agent has of the motor-tosensory mapping, i.e. of mapping between articulatory gestures $M_{V}$ (vowel), $M_{C}$ (consonant) and formant values $S_{V}$ and $S_{C}$. Once again, mappings are described by Gaussian probability distributions, where mean vectors and covariance matrices are estimated during the learning process (see below). The term $P\left(M_{C} \mid M_{V}\right)$ encodes a support for consonants that can be achieved according to the perturbation hypothesis described in the section 'Generation of stop consonants'. More specifically, for each vowel motor gesture $M_{V}, P\left(M_{C} \mid M_{V}\right)$ defines a probability distribution that is a plateau in the 5-D articulatory space for consonants. It is uniform on the possible attainments of consonants obtained by the joint use of the parameter Jaw and another one (either LipHeight for /b/, TongueApex for /d/ or TongueDorsum for /g/), and it is null everywhere else for configurations that are not consonants (because the vocal tract is not closed enough) or for configurations that cannot be reached from the $M_{V}$ vowel configuration considered. The term $P\left(M_{C} \mid M_{V}\right)$ implements a constraint coming from the physics of the Learning Agent's vocal tract (modeled by VLAM), which does not have to be estimated in the learning stage. This constraint is implemented using conditional probability tables, assigning a constant value to each achievable consonant gesture and zero probability otherwise.

The motor system describes a state of knowledge of the link between $O_{S}$ syllable labels and articulatory gestures. The structure of the motor system implements a simplified co-articulation model based on Öhman's perturbation hypothesis (Öhman, 1966). This explicitly introduces a delta variable describing the perturbation superimposed on the vowel to obtain a plosive consonant. Furthermore, we assume in COSMO-S that the Learning Agent 
1063

1064

1065

1066

1067

1068

1069

1070

1071

1072

1073

1074

1075

1076

1077

1078

1079

1080

1081

1082

1083

1084

1085

1086

1087

would have at its disposal a set of primitive consonant gestures corresponding to the basic places of articulation for plosives: combined jaw and lips for bilabials, combined jaw and tongue apex for alveolars, and combined jaw and tongue dorsum for velars. The learning process would consist of discovering these basic primitive gestures through motor exploration, and identifying their correspondence with the $\mathrm{CV}$ sequences provided by the Master Agent. Therefore, while vowels in the motor repertoire are characterized by their articulatory configuration $M_{V}^{\prime}$ (TongueBody, TongueDorsum and LipHeight in VLAM), plosives are characterized by their primitive gesture $G^{\prime}{ }_{C}$, referring to the articulator used to make a plosive consonant in coordination with Jaw (LipHeight for /b/, TongueDorsum for /g/, and TongueApex for $/ \mathrm{d} / . G_{C}^{\prime}$ is thus a categorical variable, with three possible values).

Variables $M_{V}^{\prime}$ and $G_{C}^{\prime}$ are taken to be independent. Hence

$P\left(M_{V}^{\prime} G_{C}^{\prime} \mid O_{S}\right)=P\left(M_{V}^{\prime} \mid O_{S}\right) \cdot P\left(G_{C}^{\prime} \mid O_{S}\right)$. The motor configuration of the plosive in the framework of Öhman's perturbation theory is then defined by $\Delta^{\prime}{ }_{M C}$, the variation of the articulators (the specific combination of Jaw and another specific articulator) necessary to achieve a consonant from $M_{V}^{\prime}$. The motor command for the $M^{\prime}{ }_{C}$ consonant is finally obtained by the equation $M_{C}^{\prime}=M_{V}^{\prime}+\Delta^{\prime}{ }_{M C}$. The term $P\left(\Delta^{\prime}{ }_{M C} \mid M_{V}^{\prime} G_{C}^{\prime}\right)$ describes how the consonant is produced, depending on the vowel and the specific consonant gesture. This shows explicitly that the consonant is conditioned by the vowel, which can be interpreted as anticipation. For instance, to produce the sound $/ \mathrm{ba} /$, the $/ \mathrm{a} /$ is anticipated when $/ \mathrm{b} /$ is performed, which amounts to having maximal co-articulation. $P\left(M_{V}^{\prime} \mid O_{S}\right)$ and $P\left(\Delta_{M C}^{\prime} \mid M_{V}^{\prime} G_{C}^{\prime}\right)$ are described by Gaussian distributions, where the mean vectors and covariance matrices are estimated during the learning process (see below). Finally, $P\left(G_{C}^{\prime} \mid O_{S}\right)$ is implemented with a conditional probability table (histogram), the parameters of which are also identified during learning.

The COSMO-S model is thus defined by the joint probability distribution 
1088

1089

1090

1091

1092

1093

1094

1095

1096

1097

1098

1099

1100

1101

1102

1103

1104

1106

1107

1108

1109

1110

1111

decomposition shown in Figure 12 (bottom).

Similarly to the summary of Figure 2, the Bayesian inference within the COSMO-S model allows computing of conditional probability distributions. Purely motor, purely auditory and perceptuo-motor instances of the speech perception task are implemented.

Because of the complexity of the COSMO-S model, we have not detailed the corresponding Bayesian inferences here. However, they can be interpreted exactly as previously: auditory perception is expressed as direct use of the link between auditory representations and the corresponding object labels, motor perception as the combination of the motor repertoire with an internal model allowing association of motor and sensory representations, and perceptuomotor perception as the Bayesian fusion of the auditory and motor categorization processes. We will now describe how the Learning Agent acquires the different parts of the model.

\section{Learning in COSMO-S}

Some probability distributions of the model are not learned. Indeed, the prior $P\left(O_{S}^{A g}\right)$, $P\left(O_{L}^{A g}\right)$ and $P\left(M_{V}^{A g}\right)$ are set as uniform probability distributions. The biological constraints $P\left(M_{C}^{A g} \mid M_{V}^{A g}\right)$ describe the consonants achievable from a given vowel, and are pre-computed in $V L A M$. Finally, probability distributions over coherence variables, $P\left(\lambda_{S V}^{A g} \mid S_{V}^{A g} S_{V}^{\prime A g}\right)$, $P\left(\lambda_{S C}^{A g} \mid S_{C}^{A g} S_{C}^{\prime A g}\right), P\left(\lambda_{M V}^{A g} \mid M_{V}^{A g} M_{V}^{\prime A g}\right), P\left(\lambda_{M C}^{A g} \mid M_{C}^{A g} \Delta_{M C}^{\prime A g} M_{V}^{\prime A g}\right)$ and $P\left(C^{A g} \mid O_{S}^{A g} O_{L}^{A g}\right)$ are set as Dirac probability distributions, with True value of a probability of 1 for a given relationship between the variables on the right hand side, respectively $S_{V}^{A g}=S_{V}^{\prime A g}, S_{C}^{A g}=$ $S_{C}^{\prime A g}, M_{V}^{A g}=M_{V}^{\prime A g}, M_{C}^{A g}=\Delta_{M C}^{\prime A g}+M_{V}^{\prime A g}$ and $O_{S}^{A g}=O_{L}^{A g}$

The probability distributions that the Learning Agent apprehends in COSMO-S are the same as in the 1-D implementation studied in the previous section: the auditory categorization branch $P\left(O_{L}^{A g} \mid S_{V}^{A g} S_{C}^{A g}\right)$, the forward model implementing the motor-to-auditory 
1112 relationship $P\left(S_{V}^{A g} S_{C}^{A g} \mid M_{V}^{A g} M_{C}^{A g}\right)$ and the motor repertoire $P\left(M_{V}^{A g} M_{C}^{A g} \mid O_{S}^{A g}\right)$. As

1113 previously, we learn the auditory and motor branches independently from each other, but with

1114 the same set of data. This allows a fair comparison between the two branches.

1115 While the forward model and the motor repertoire were learned in 1-D, a two-stage

1116 process was implemented in COSMO-S. Indeed, considering the complexity of the motor-to-

1117 auditory relationship within a 12-D space ( 8 motor plus 4 auditory dimensions), it appeared

1118 easier to learn the forward model before the motor repertoire. This corresponds well to the

1119 developmental schedule presented previously (Kuhl, 2004), which led us to proceed in three

1120 consecutive steps:

1121 L1. learning the auditory categories;

1122 L2. learning motor-to-auditory mapping;

1123 L3. learning the motor repertoire.

1124 During these three learning phases, the Learning Agent interacts with a Master Agent 1125 to obtain syllable acoustic stimuli $(F 2, F 3$ for the plosive, $F 1, F 2$ for the vowel) taken from 1126 the data displayed in Figure 10 and, for steps L1 and L3, the corresponding syllable labels as 1127 well. Phases L2 and L3 are independent of phase L1; hence they will be evaluated separately 1128 in the following argument.

\section{L1: Learning the auditory categories by association.}

$1132 S_{V}^{\prime A g}$ and $S_{C}^{A g}$ and corresponding syllables $O_{L}^{A g}$, is learned by association, through interactions

1133 with the Master Agent. More precisely, the term $P\left({S^{\prime}}_{V}^{A g}{ }_{S_{C}^{\prime}}^{A g} \mid O_{L}^{A g}\right)$ consists of 9 auditory

1134 prototypes (one for each value of $O_{L}^{A g}$ ) encoded as 4-D Gaussian probability distributions on

1135 the formant space $\left(F 1_{V}, F 2_{V}, F 2_{C}, F 3_{C}\right)$, which the agent learns in a supervised manner from 1136 the Master Agent. This provides < formant values, syllable label $>$ pairs. Auditory recognition 
1137

1142

1144 available, it selects motor gestures randomly. New observations lead to improving the quality $P\left(O_{L}^{A g} \mid{S^{\prime}}_{V}^{A g} S_{C}^{\prime A g}\right)$ is then implemented by the Bayesian inversion of $P\left({S^{\prime}}_{V}^{A g} S_{C}^{A g} \mid O_{L}^{A g}\right)$ :

$$
P\left(O_{L}^{A g} \mid S_{V}^{\prime A g} S_{C}^{\prime A g}\right)=\frac{P\left(S_{V}^{A g} S_{C}^{A g} \mid O_{L}^{A g}\right)}{\sum_{o} P\left(S_{V}^{A g} S_{C}^{A g} \mid\left[O_{L}^{A g}=o\right]\right)}
$$

\section{L2: Learning the motor-to-auditory mapping by accommodation.}

Since we attempt to learn the sensory-motor system independently of the motor repertoire, learning is achieved by a variant of the learning by accommodation algorithm, in which the Learning Agent only obtains auditory input from the Master Agent, without object labels. Given a syllable acoustic target $\left(s_{V}, s_{C}\right)$, and using its current state of knowledge as given by $P\left(S_{V}^{A g} S_{C}^{A g} \mid M_{V}^{A g} M_{C}^{A g}\right)$, the Learning Agent carries out imitation tasks, by inferring a motor gesture $\left(m_{V}, m_{C}\right)$ likely to reach the target. This gesture is obtained by randomly drawing a value $\left(m_{V}, m_{C}\right)$ according to the inversion of the current forward model:

$$
\begin{gathered}
P\left(M_{C}^{A g} M_{V}^{A g} \mid\left[S_{V}^{A g}=s_{V}\right]\left[S_{C}^{A g}=s_{C}\right]\right) \propto \\
P\left(M_{V}^{A g}\right) \cdot P\left(\left[S_{V}^{A g}=s_{V}\right] \mid M_{V}^{A g}\right) \cdot P\left(M_{C}^{A g} \mid M_{V}^{A g}\right) \cdot P\left(\left[S_{C}^{A g}=s_{C}\right] \mid M_{C}^{A g}\right) .
\end{gathered}
$$

The gesture $\left(m_{V}, m_{C}\right)$ is sent to $V L A M$, which plays the role of an external vocal tract simulator. $V L A M$ outputs the formants $\left(s_{V}^{*}, s_{C}^{*}\right)$ corresponding to the motor command $\left(m_{V}, m_{C}\right)$, and the Learning Agent updates the knowledge stored in its internal models. It observes that the chosen motor commands produce a given set of formants. This knowledge is stored in the probability distributions $P\left(S_{V}^{A g} \mid\left[M_{V}^{A g}=m_{V}\right]\right)$ and $P\left(S_{C}^{A g} \mid\left[M_{C}^{A g}=m_{C}\right]\right)$, which are Gaussian probability distributions evolving as their parameters become updated through the learning process.

The syllable targets provided by the Master Agent to the Learning Agent are taken from the data presented in Figure 10. Since the Learning Agent initially has no knowledge of the internal model of the motor-to-auditory transformation, which in turn improves the 
1161

1162

1163

1164

motor inversion that relies on this internal model. This means that the computed probability distribution $P\left(M_{C}^{A g} M_{V}^{A g} \mid\left[S_{V}^{A g}=S_{V}\right]\left[S_{C}^{A g}=s_{C}\right]\right)$ driving the choice of motor gestures and allowing imitation of auditory inputs becomes more and more accurate. Thus, the agent becomes better and better at reaching its targets. All along the exploration process, the learning algorithm remains driven by the targets provided by the Master, rather than by an exhaustive sampling of the motor space as in other systems (e.g., Bailly, 1997; Guenther, 2006).

\section{L3: Learning the motor repertoire by imitation.}

The motor system is learned in a supervised way, in that syllable labels are given to the Learning Agent along with the corresponding stimuli. But while in other research the articulatory data are provided (Castellini et al., 2011; Canevari et al., 2013), here the Learning Agent is only given labeled acoustic data. We use the same $<$ formant values, syllable label $>$ pairs that served to learn auditory categorization in step L1, and we use the internal model of the motor-to-auditory transformation learned in step L2 to retrieve motor information. Given an acoustic target $\left(s_{V}, s_{C}\right)$ and the corresponding syllable label $o_{S}$, the Learning Agent infers a motor gesture allowing the target to be reached by inversing the motor-to-auditory mapping and by using its present state of knowledge of the correspondence between syllables and motor gestures. This is done by randomly drawing $\left(m_{V}^{\prime}, g_{C}^{\prime}, \delta^{\prime}{ }_{M C}\right)$ values according to the following probability distribution:

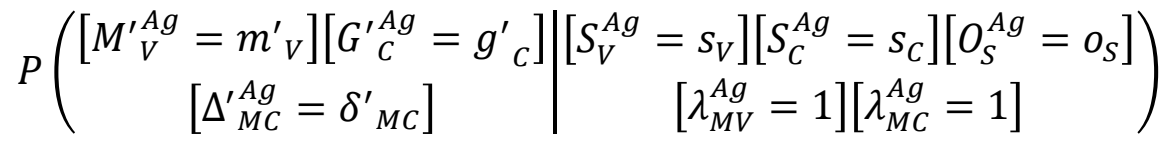

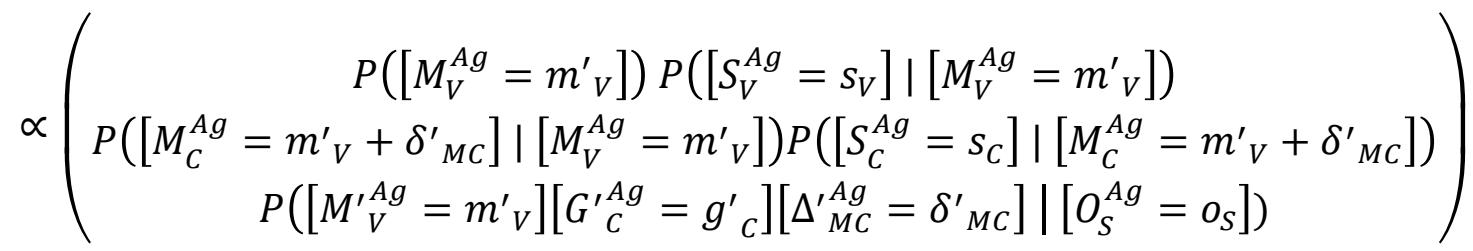


The correspondence between the chosen motor gesture $\left(m_{V}^{\prime}, g_{C}^{\prime}, \delta^{\prime}{ }_{M C}\right)$ and the

1182

1183 syllable label $o_{S}$ is then used to update parameters of the following probability elements: the Gaussian probability distribution $P\left(M_{V}^{\prime A g} \mid O_{S}^{A g}\right)$, the histogram $P\left(G_{C}^{\prime A g} \mid O_{S}^{A g}\right)$ and the Gaussian probability distribution $P\left(\Delta_{M C}^{\prime A g} \mid M_{V}^{\prime A g} G_{C}^{\prime A g}\right)$.

\section{Simulation results}

\section{Confirming the "auditory-narrowband vs. motor-wideband" portrait in COSMO-} $S$.

The aim of the simulations described in this section is to verify how the main principles we extracted from the results of the experiments carried out in the 1-D case are generalized to the more realistic case of syllable processing. We ran a single learning simulation with 4,000,000 < formant values, syllable label $>$ pairs. The first 3,000,000 were used during the L2 learning phase, with another 1,000,000 during L3 and the full set of the same 4,000,000 values were also used for the L1 learning phase. These were resampled from the data presented in Figure 10 and provided to the Learning Agent by the Master Agent. Since the L1 learning phase of the sensory model is independent from the L2 and L3 learning phases of the perceptuo-motor and motor models and for future comparisons of the auditory and motor models of perception to be fair, the same data is used as input to learn the components involved in motor and auditory perception.

\section{***FIGURE 13 ABOUT HERE***}

Firstly, in Figure 13 we compare the evolution through learning of the entropy

$H\left(P\left(S^{A g} \mid O_{L}^{A g}\right)\right)$ of the auditory model, with the evolution of the entropy $H\left(P\left(S^{A g} \mid O_{S}^{A g}\right)\right)$ of the motor model. To this end, as in Figure 5 we used the entropy $H\left(P\left(S^{A g} \mid O_{S}^{\text {Master }}\right)\right)$ of the probability distribution over the stimuli produced by the Master Agent, a constant over the learning process, as a reference. As in the 1-D case, we further average these entropies over 
1206

1207

1208

1211

1212

1213

1214

1215

1216

1217

1218

1219

objects; since we now have 9 possible objects, we consider $\frac{1}{9} \sum_{O_{L}^{A g}} H\left(P\left(S^{A g} \mid O_{L}^{A g}\right)\right)$ for the auditory model, $\frac{1}{9} \sum_{O_{S}^{A g}} H\left(P\left(S^{A g} \mid O_{S}^{A g}\right)\right)$ for the motor model, and

$\frac{1}{9} \sum_{O_{S}^{M a s t e r}} H\left(P\left(S^{A g} \mid O_{S}^{\text {Master }}\right)\right)$ as the Master's reference.

We observe that, as with the 1-D model, the entropy of the auditory model converges quickly to a level close to the entropy of the stimuli produced by the Master, whereas the entropy of the motor model converges more slowly ${ }^{(6)}$.

***FIGURE 14 ABOUT HERE***

To better display how exploration and learning proceed in COSMO-S, we show in Figure 14 the motor gestures $m^{\prime}{ }_{V}$ selected by the Learning Agent to attempt to reproduce the auditory targets provided by the Master Agent at five stages in the learning process: before any learning took place, during the L2 learning phase, between L2 and L3, during L3, and at the end of the learning process. We observe that learning enables progressive focusing of the vowel articulatory gestures around given areas in the articulatory space, corresponding to adequate commands for the three vowels /a, i, u/, but the size of the possible motor configurations remains wide.

\section{***FIGURE 15 ABOUT HERE***}

Comparatively, we display in Figure 15 the actual productions of the Learning Agent in acoustic space at the same five steps in the learning process: the size of the available auditory space is more reduced around the three vowels /a i $\mathrm{u} /$ provided by the Master Agent at the end of the learning process.

***FIGURE 16 ABOUT HERE***

To evaluate the global categorization ability of the auditory, motor and perceptuomotor branches in COSMO-S at the end of the learning process with 4,000,000 iterations, we exploited the same methodology as with the 1-D implementation of COSMO. We took as 
1230 input the formant values $(F 2, F 3$ for the consonant, $F 1, F 2$ for the vowel) produced by the

1231 Master Agent (and displayed in Figure 10). We added a given level of noise by adding a

1232 Gaussian perturbation to each formant value, with a given variance indexed by the noise level.

1233 We present these stimuli to the auditory, motor, or perceptuo-motor classifier defined in

$1234 \mathrm{COSMO}-\mathrm{S}$ according to the equations derived from Figure 2. In practice, we used exact

1235 inferences for evaluation: stimuli were not sampled from the Master Agent and then decoded,

1236 rather the whole probability distribution of stimuli was used to directly compute the resulting

1237 confusion matrices for each classifier. The original object $O_{S}^{\text {Master }}$ was compared with the

1238 decoded object $O_{L}^{\text {Agent }}$ or $O_{S}^{\text {Agent }}$ depending on the model considered. Average diagonal

1239 values of the confusion matrices provide recognition scores that are displayed in Figure 16.

1240 The pattern of results is similar to that obtained with 1-D simulations. For clean

1241 stimuli (Figure 16, noise $=0$ ), the auditory model is more accurate than the motor one. The

1242 difference is small, considering the large difference in entropies at the end of the learning

1243 process (see Figure 13), but this is because the distributions to categorize are well separated in

1244 these simulations. The difference would be larger in less clean learning configurations. When

1245 noise is added, the motor system performance decreases less rapidly than the auditory one,

1246 and it becomes more accurate for noise levels greater than 0.5 . The perceptuo-motor model

1247 capitalizes on the fusion of the two branches to provide better scores than the separate

1248 auditory and motor models, at all noise levels.

\section{Assessing auditory vs. motor invariance for the place of articulation of vowels}

1250 and plosives in COSMO-S.

1251 We now explore our second proposal about auditory-motor complementarity,

1252 assessing how phonemic invariance could be represented in the auditory or motor branches in

1253 COSMO-S.

1254 The situation for vowels has already been presented in Figures 14 and 15. It is in 
1255

1256

agreement with our predictions; while the acoustic characterization of vowels is rather straightforward (see the final learning stage in Figure 15), the distribution of motor variables is more disordered (see the final learning stage in Figure 14).

\section{***FIGURE 17 ABOUT HERE***}

For plosives, the acoustic configuration is more complicated than for vowels. Indeed, Figure 10 shows how intricate the formant configurations are for each plosive, due to vowel co-articulation. This is where the motor system could play a crucial role. Indeed, in Figure 17 we display the evolution of the motor variable $P\left({G_{C}^{\prime}}_{C}^{A g} \mid O_{S}^{A g}\right)$ distribution for the 9 objects $O_{S}^{A g}$. Each subplot displays the evolution of the probabilities of the three possible gestures (LipHeight, TongueDorsum and TongueApex) for each object at each learning stage. It appears that for 8 cases out of 9 , the identification of the correct gesture has been successful: LipHeight for /ba, bi, bu/, TongueDorsum for/ga, gi, gu/ and TongueApex for/da, di, du/. This means that the Learning Agent has selected a gesture compatible with that performed by the Master Agent, and that motor invariance is within reach through the existence of the ${G^{\prime}}_{C}^{A g}$ parameter in the motor repertoire.

However, there is one case where adequate identification has partly failed: for the object /gi/, the probability of the TongueApex gesture remains high, even though this is not the adequate gesture for the velar in /gi/. The reason is clear: looking at Figure 10, it can be seen that the acoustic regions for /di/ and for /gi/ are partially superimposed. This is probably due to an acoustic description of the plosives that is too simplified (e.g. lacking higher formants, burst fine characteristics or spectral dynamics, which could all play a part in improving the /di/-/gi/ contrast). However, even if there was an articulatory ambiguity, we may suppose that hyper-articulation by the Master Agent could guide the Learning Agent to solve the problem. In a further simulation, we implemented this process by having a Master Agent discarding productions before reaching an acoustic zone where both /di/ and /gi/ could be produced. With 
1280 $1281 \quad$ Figure 18.

1282

1283

1284

1285

1286

1287

1288

1289

1290

1291

1292

1293

1294

1295

1296

1297

1298

1299

1300

1301

1302

1303

1304

such a dataset for /gi/ production, the recovery of adequate gestures is perfect, as displayed in

***FIGURE 18 ABOUT HERE***

\section{Concluding Part 3: Summary and predictions}

The simulations with COSMO-S enable a significant gap in complexity to be crossed and the possibility of implementing and testing the COSMO model in a high dimension (8 articulatory +4 acoustic parameters) could be assessed. These provide two major results. Firstly, we confirmed the auditory-narrow motor-wide portrait introduced in Part 2.

Once again we obtain both quicker and more efficient learning of acoustic stimuli in the sensory compared with the motor pathway (Figures 13-15), resulting in better auditory recognition scores than motor ones without noise, but also a superiority of the motor decoding process in noisy conditions (Figure 16). In consequence, altogether the perceptuo-motor model performs better than both the auditory and the motor pathways whatever the noise level (Figure 16). This was expected, given that the auditory-narrow motor-wide hypothesis is considered to be generic and independent of the underlying specific implementation.

However, it is of interest to confirm that it is displayed in a much more complex and realistic sensory-motor environment in COSMO-S compared with the 1-D simulations of Part 2. Interestingly, the motor-to-sensory transformation in COSMO-S, associated with VLAM, is no longer monotonous. Altogether, and unsurprisingly, the increase in complexity even results in a much larger difference in learning speed and efficiency between auditory and motor inference in COSMO-S compared with 1-D simulations (compare Figures 5 and 13).

Secondly, the analysis of the information content of auditory vs. motor representations lets a natural complementarity appear, with plosives on one side, difficult to characterize in the auditory space, but clearly associated with a motor or gestural invariant in the motor 
1305 repertoire, and vowels on the other side, for which the auditory characterization is more

1306 efficient than the motor one.

1307 The potential limitations of this study are related to the nature of the hypotheses we

1308 introduced to make the implementation of tractable simulations possible. Firstly, we had to

1309 base our work on artificial synthetic CV sequences. Indeed, COSMO requires a sensory-motor

1310 model able to process stimuli characterized in the motor (articulatory) and sensory (acoustic)

1311 spaces. No currently available speech production model can produce completely realistic

1312 speech stimuli. Thus we needed to restrict our simulations to synthetic stimuli generated by

1313 the model we had at our disposal, i.e. VLAM. However, the realism of formant data in our

1314 simulations, and the relative complexity of the material that we provided for processing in

1315 COSMO-S, make us confident that real speech is not out of reach of COSMO development.

1316 A second hypothesis in COSMO-S is the assumption that the Learning Agent has at its

1317 disposal a set of primitive coordination gestures, i.e. Jaw/Lips, Jaw/Tongue Apex or

1318 Jaw/Tongue Dorsum, corresponding respectively to labial, alveolar and velar places of

1319 articulation. This hypothesis deserves further comment. It is consistent with data on infant

1320 imitation showing that infants have at their disposal basic facial gestures that they can identify

1321 from birth on the face of their communicating partner (Meltzoff \& Moore, 1977). The

1322 Articulatory Organ Hypothesis developed in the Haskins Labs at the beginning of the 2000s

1323 (see e.g., Best \& McRoberts, 2003; Goldstein \& Fowler, 2003) exploits precisely this kind of

1324 assumption to describe perception and control in the framework of Articulatory Phonology. It

1325 supposes that infants are able to detect in a speech signal the primary articulatory organ that

1326 produced it. Current simulations provide this hypothesis with some computational basis.

1327 These two results, "auditory-narrow, motor-wide" and "auditory-vowel, motor-

1328 plosive" provide two major sets of experimental predictions. 
Prediction 3 - early speech perception should be mostly auditory before the onset

\section{of babbling, then become progressively perceptuo-motor}

The PACT proposal is that speech perception should make use of only the auditory pathway in the first months of age, then progressively capitalize on feedback from the motor system when it is mature and mainly since babbling onset around 7 months. This appears to be reinforced in the context of the auditory-narrow, motor-wide hypothesis. The learning pattern in Figure 13 strongly confirms the view that auditory perception should be mature long before motor information could be used for phonetic decoding. Considering the potential role of the motor system for perception in noisy conditions, it could be suggested that as this is still immature at the first months of age, it should not intervene specifically in adverse conditions, before some significant degree of sensory-motor development. Basically, it is after babbling onset that infants can obtain a useful amount of information on the motor inference branch.

This is exactly what was described in a recent MEG (Magnetoencephalography) study on infants' brain responses to native vs. non-native stimuli at two developmental stages in the first year of age (Kuhl et al., 2014). Indeed, the data in this study showed that infants at 7 months of age do not display a significantly different involvement of the motor regions (including Broca's area and the cerebellum) for native vs. non-native speech. In contrast, at 11-12 months of age, i.e. after a significant amount of perceptuo-motor learning has occurred, following babbling onset at around 7 months, there is more involvement of motor regions for non-native compared with native stimuli.

\section{Prediction 4 - plosive place of articulation invariance should require motor}

\section{knowledge}

The last prediction is related to the second result obtained with COSMO-S about the 
"auditory-vowel, motor-plosive" hypothesis. The corresponding results in COSMO-S suggest that the identification of invariant cues for the plosive place of articulation should strongly depend on the acquisition of motor representations associated with sensory input in the motor inference process.

As mentioned in the Introduction, a number of experimental data do indeed suggest that infants cannot detect the plosive place of articulation invariance before 6 months of age. A recent study by Hochmann \& Papeo (2014) exploiting a novel methodology based on pupillometry provided a hint that the "b" vs. " $\mathrm{d}$ " contrast could be displayed independently in vowel context at 6 months. However, auditory and visual information could be at the basis of this result (Dole et al., 2015). Importantly, another recent study in our group, exploiting an inter-sensory matching procedure, provided different results compatible with the present prediction. This procedure provided no evidence for articulation plosive identification independent of vowel context at 6 months of age, but some such evidence was seen at 9 months. Importantly, infants' perceptual abilities appeared to be related to their motor abilities in babbling (Dole, Loevenbruck, Pascalis, Schwartz, \& Vilain, 2016).

The present prediction should not be generalized to the proposal that there would be no involvement at all of the motor system in speech perception before babbling onset. Indeed, Bruderer, Danielson, Kandhadai \& Werker (2015) demonstrated that teething displays used to control infants' tongues in their mouths may interfere with the perception of non-native stimuli related to the corresponding induced tongue shapes for 6 month old subjects. The important point of our prediction is that the plosive place of articulation requires learning the sensory-to-motor correspondence in complete $\mathrm{CV}$ sequences that are out of reach before the onset of babbling.

Finally, it is of interest that a recent analysis of fMRI responses to CV syllables using multivariate decoding shows that plosive place of articulation is indeed specifically found to 
1380 be represented in regions of the brain associated with speech production, including the

1381 posterior ventral frontal cortex, the basal ganglia, and the cerebellum (Correia, Jansma \& 1382 Bonte, 2015).

1383 


\section{Part 4 - Three challenges for a perceptuo-motor theory of speech perception} model of speech perception, COSMO, together with various implementations (from 1-D to COSMO-S). Furthermore, we have two major results about "non-perfect" learning conditions, enabling to depart from the indistinguishability theorem: the "auditory-narrow, motor-wide" and "auditory-vowel, motor-plosive" properties. This model opens a number of perspectives

1391 for future developments. We will discuss three major directions for research in the field of perceptuo-motor interactions involving potential developments in COSMO.

\section{Challenge 1: Perceptuo-motor complementarity and Perceptuo-motor fusion} functional arguments about the auditory vs. motor controversy, somewhat lacking of experimental data, to convincing experimental neurocognitive data supporting the role of the motor system, but somewhat lacking of functionalist views about why the motor system could

1399 be useful at all. The present study attempted to provide such functionalist arguments. Future

1400 studies should attempt to provide more data about when, how and why the motor system

1401 could enhance auditory perception. Furthermore, a perceptuo-motor theory of speech

1402 perception requires a fusion process enabling efficient combination of auditory (if not visual

1403 or somatosensory) and motor information for speech decoding.

1404 Interestingly, audiovisual speech perception research has asked more or less the same 1405 questions for about the last forty years. It was shown how auditory and visual inputs could be 1406 complementary to a certain extent (e.g. Summerfield, 1987; Robert-Ribes, Schwartz,

1407 Lallouache, \& Escudier, 1998). Audio-visual fusion led to many theoretical and 1408 methodological developments, proposing that it could be optimal in the Bayesian sense (see 
1409 Massaro and the Fuzzy-Logical Model of Perception, 1987, 1998; in relation with Ernst \&

1410 Banks, 2002), and that within fusion each sensory modality could possibly be weighted

1411 according to its reliability, depending on context, language, subjects, etc. (Schwartz, 2010).

1412 Perceptuo-motor complementarity and fusion should thus be set at a high position in

1413 the research agenda on perceptuo-motor speech perception, just as they were in past research

1414 on audiovisual speech perception.

1415

1416 Challenge 2 - Integrating speech perception and speech production in a common

\section{7 framework}

1418 Accumulating evidence for the role of motor knowledge in speech perception may be

1419 combined with accumulating evidence for the role of perceptual representations and processes

1420 in speech motor control (see reviews in Guenther, Hampson \& Johnson, 1998; Perrier, 2005).

1421 Importantly, the current perceptuo-motor model of speech perception capitalizes on a set of

1422 computational bricks traditionally involved in speech production models. Thus, sensory and

1423 motor representations are associated thanks to internal forward or inverse models (e.g.

1424 Guenther, Ghosh \& Tourville, 2006; Houde \& Nagarajan, 2011; Hickok, 2012; Patri, Diard \& 1425 Perrier, 2015).

This suggests that it could be possible to develop an integrated framework associating

1427 speech perception and speech production models within the same theoretical architecture.

1428 This is one aim of the COSMO architecture (see Moulin-Frier et al., 2012, 2015).

1429 Interestingly, the same objective has been introduced in recent phonological models (see e.g.

1430 Boersma, 2011, Boersma \& Hamman, 2008).

1432 Challenge 3 - From computational architecture to neurocognitive implementation

1433 It is widely acknowledged, at least since Marr (1982), that cognitive systems can be 
1434 analyzed at different levels, three in Marr's proposal: computational, algorithmic, and

1435 representational and implementation levels. These levels are independent to a certain extent,

1436 but the computational and algorithmic architectures may shed light on the way neurocognitive

1437 implementation could be realized. Conversely, neurocognitive constraints could suggest some

1438 proposals for algorithmic considerations.

1439 At this stage, we did not elaborate in any way the possible neurocognitive means by

1440 which the various components in COSMO could be implemented in the human brain. This is

1441 not to say that such an enterprise, relating computation and implementation levels, is out of

1442 reach, as was clearly displayed by the authors of the DIVA model of speech production

1443 (Guenther et al., 2006). Considering the increasing amount of details provided by

1444 neuroscience about the neural coding of speech perception and production in the auditory and

1445 motor cortex (see e.g. Bouchard, Mesgarani, Johnson \& Chang, 2013; Cheung, Hamiton,

1446 Johnson, \& Chang, 2016; Formisano, De, Bonte, \& Goebel, 2008; Pasley et al., 2012), it is

1447 now a challenging but intriguing and probably necessary enterprise to attempt to elaborate

1448 further the possible relationships between computational models such as COSMO and neural

1449 responses in a number of experimental tasks.

\section{Conclusion}

This paper develops an original perspective in the debate between auditory and motor

1453 theories of speech perception. Research in the cognitive neurosciences led to the now well-

1454 accepted views that (i) motor areas are activated during speech perception, and (ii) motor

1455 knowledge seems to play a certain role in speech perceptual processing in the human brain.

1456 From these points of view, we attempted to evaluate the precise functional role of motor

1457 knowledge. In the framework of PACT, a perceptuo-motor theory of speech perception, we

1458 explored these questions in computational terms, thanks to COSMO, the first Bayesian 
1459

1460

1461

1462

perceptuo-motor model of speech communication.

We showed here for the first time that, in conditions that are perfect in a certain sense, the information content of the auditory and motor branches of a perceptuo-motor speech processing system are exactly the same. We introduced realistic learning conditions and showed that they let a natural complementarity emerge between a "narrow-band" auditory system that is more efficient in good communication conditions, and a "wide-band" motor system that is more efficient in adverse conditions. Our simulations also suggest that invariants providing the phonetic characterization of phonological units could be perceptuomotor rather than auditory or motor, and show how this could be achieved, with auditory cues for vowels and motor cues for the plosive place of articulation.

COSMO simulations lead to a number of experimental predictions. Some of these are already being tested, with data in agreement with predictions. Others require more experimental efforts. COSMO also opens a number of perspectives in domains such as: the fusion of perceptual and motor inference in phonetic decoding; the co-development of computational models of speech production and speech perception; the possibility to apply COSMO simulations to a number of neurocognitive data on the coding and processing of speech in the human brain.

This research has placed computational simulations at the heart of the debate about the role of perceptual and motor knowledge in the speech perception process. Considering the rapidly increasing amount of experimental evidence and data available about the perceptuomotor relationship in speech communication, it seems that mathematical models can be of great help in clarifying arguments, precising mechanisms and suggesting new predictions and experimental paradigms. Perceptuo-motor complementarity, invariance, fusion and development are crucial steps in the agenda of future research into the cognitive bases of speech communication. The first pieces in the elaboration of the COSMO model described 
1484 and discussed in the present paper provide convincing elements for pursuing this direction. 
References

1486 Ashby, F. G., \& Townsend, J. T. (1986). Varieties of perceptual independence. Psychological Review, 93, 154-179. http://dx.doi.org/10.1037/0033-295X.93.2.154

Ashby, F. G., \& Perrin, N. A. (1988). Toward a unified theory of similarity and recognition. Psychological Review, 95, 124-150. http://dx.doi.org/10.1037/0033-295X.95.1.124

1490 d'Ausilio, A., Bufalari, I., Salmas, P., \& Fadiga, L. (2012). The role of the motor system in discriminating normal and degraded speech sounds. Cortex, 48, 882-887. http://dx.doi.org/10.1016/j.cortex.2011.05.017

d'Ausilio, A., Pulvermüller, F., Salmas, P., Bufalari, I., Begliomini, C., \& Fadiga, L. (2009). The motor somatotopy of speech perception. Current Biology, 19, 381-385. http://dx.doi.org/10.1016/j.cub.2009.01.017

Badin, P. \& Fant, G. (1984). Notes on Vocal Tract Computation. In Quarterly Progress and Status Report, Dept. for Speech, Music and Hearing, KTH, Stockholm (pp. 53-108).

Bailly, G. (1997). Learning to speak. Sensori-motor control of speech movements. Speech Communication, 22, 251-267. http://dx.doi.org/10.1016/S0167-6393(97)00025-3

1500 Barnaud, M.L., Schwartz, J.L., Diard, J., \& Bessière, P. (2016). Sensorimotor learning in a Bayesian computational model of speech communication. 6th International Conference on Development and Learning and on Epigenetic Robotics.

Basirat, A., Schwartz, J.-L., \& Sato, M. (2012). Perceptuo-motor interactions in the perceptual organization of speech: Evidence from the verbal transformation effect. Philosophical Transactions of the Royal Society B: Biological Sciences, 367, 965-976. http://dx.doi.org/10.1098/rstb.2011.0374 short CVs. Journal of the Acoustical Society of America, 82, 31-37. 
1510 Bertoncini, J., Bijeljac-Babic, R., Jusczyk, P. W., Kennedy, L. J., \& Mehler, J. (1988). An investigation of young infants' perceptual representations of speech sounds. Journal of Experimental Psychology: General, 117, 21-33. http://dx.doi.org/10.1037/0096-3445.117.1.21

1513

1514

Bessière, P., Laugier, C., \& Siegwart, R. (2008). Probabilistic reasoning and decision making in sensory-motor systems. Springer Tracts in Advanced Robotics. Berlin: Springer-Verlag.

Bessière, P., Mazer, E., Ahuactzin-Larios, J.-M., \& Mekhnacha, K. (2013). Bayesian Programming. CRC Press. Boca Raton, FL.

Best, C.C., \& McRoberts, G.W. (2003). Infant Perception of Non-Native Consonant Contrasts that Adults Assimilate in Different Ways. Language and Speech, 46, 183-216. http://dx.doi.org/doi:10.1177/00238309030460020701

Bever, T.G., \& Poeppel, D. (2010). Analysis by Synthesis: A (Re-)Emerging Program of Research for Language and Vision. Biolinguistics, 4.2-3, 174-200. http://www.biolinguistics.eu

Binder, J.R., Liebenthal, E., Possing, E.T., Medler, D.A., \& Ward, B.D. (2004). Neural correlates of sensory and decision processes in auditory object identification. Nature Neuroscience, 7, 295301. http://dx.doi.org/doi:10.1038/nn1198

Blumstein, S.E., \& Stevens, K.N. (1979). Acoustic invariance in speech production: Evidence from measurements of the spectral characteristics of stop consonants. Journal of the Acoustical Society of America, 66, 1001-1017. http://dx.doi.org/10.1121/1.383319

Boë, L.-J., Badin, P., Ménard, L., Captier, G., Davis, B., MacNeilage, P., Sawallis, T., \& Schwartz, J.L. (2013). Anatomy and control of the developing human vocal tract: A response to Lieberman. Journal of Phonetics, 41, 379-392.

Boë , L.-J., Perrier, P., \& Bailly, G. (1992). The geometric vocal tract variables controlled for vowel production: Proposals for constraining acoustic-to-articulatory inversion. Journal of Phonetics, $20,27-38$.

de Boer, B., \& Kuhl, P. K. (2003). Investigating the role of infant-directed speech with a computer 
model. Acoustics Research Letters Online, 4, 129-134. http://dx.doi.org/10.1121/1.1613311

1536 Boersma, P. (2011). A programme for bidirectional phonology and phonetics and their acquisition and evolution. In Anton Benz \& Jason Mattausch (Eds.) Bidirectional Optimality Theory, pp. 3372. Amsterdam: John Benjamins.

Boersma, P., \& Hamann, S. (2008). The evolution of auditory dispersion in bidirectional constraint grammar. Phonology, 25, 217-270. http://dx.doi.org/10.1017/S0952675708001474

Bouchard, K.E., Mesgarani, N., Johnson, K., \& Chang E.F. (2013). Functional organization of human sensorimotor cortex for speech articulation. Nature, 495, 327-332. http://dx.doi.org/10.1038/nature11911

de Boysson-Bardies, B. (1993). Ontogeny of language-specific syllabic production. In B. de BoyssonBardies \& S. de Schoen \& P. Jusczyk \& P. F. MacNeilage \& J. Morton (Eds.), Developmental neurocognition: Speech and face processing in the first year of life (pp. 353-363). Dordrecht: Kluwer Academic Publishers.

de Boysson-Bardies, B., Halle, P., Sagart, L., \& Durand, C. (1989). A crosslinguistic investigation of vowel formants in babbling. Journal of Child Language, 16, 1-17.

de Boysson-Bardies, B., Sagart, L., \& Durant, C. (1984). Discernible differences in the babbling of infants according to target language. Journal of Child Language, 11, 1-15.

Bruderer, A.G., Danielson, D.K., Kandhadai, P., \& Werker, J.F. (2015). Sensorimotor influence on speech perception in infancy. Proc Natl Acad Sc, 112, 13531-13536. http://dx.doi.org/10.1073/pnas.1508631112

Callan, D.E., Callan, A.M., \& Jones J.A. (2014). Speech motor brain regions are differentially recruited during perception of native and foreign-accented phonemes for first and second language listeners. Frontiers in Neuroscience, 03 September 2014, http://dx.doi.org/10.3389/fnins.2014.00275

Callan, D.E., Jones, J.A., Callan, A.M., \& Akahane-Yamada, R. (2004). Phonetic perceptual 
identification by native- and second-language speakers differentially activates brain regions involved with acoustic phonetic processing and those involved with articulatoryauditory/orosensory internal models. NeuroImage, 22, 1182-1194, http://dx.doi.org/doi:10.1016/j.neuroimage.2004.03.006

Canevari, C., Badino, L., D'Ausilio, A., Fadiga, L., \& Metta, G. (2013). Modeling speech imitation and ecological learning of auditory-motor maps. Frontiers in Psychology, 4, 364, http://dx.doi.org/10.3389/fpsyg.2013.00364

Castellini, C., Badino, L., Metta, G., Sandini, G., Tavella, M., Grimaldi, M., \& Fadiga, L. (2011). The use of phonetic motor invariants can improve automatic phoneme discrimination. PLOS ONE, 6, e24055. http://dx.doi.org/10.1371/journal.pone.0024055

Cheung, C., Hamiton, L.S., Johnson, K., \& Chang, E. F. (2016). The auditory representation of speech sounds in human motor cortex. eLife, 5:e12577. DOI: 10.7554/eLife.12577

Clayards, M., Aslin, R. N., Tanenhaus, M. K., \& Jacobs, R. A. (2007). Within category phonetic variability affects perceptual uncertainty. In Proc. 16th International Congress of Phonetic Sciences, Saarbrücken, Germany, pages 701-704.

Clayards, M., Tanenhaus, M. K., Aslin, R. N., \& Jacobs, R. A. (2008). Perception of speech reflects optimal use of probabilistic speech cues. Cognition, 108, 804-809.

Correia, J.L., Jansma, B.M.B., \& Bonte, M. (2015). Decoding articulatory features from fMRI responses in dorsal speech regions. The Journal of Neuroscience, 35, 15015-15025. http://dx.doi.org/10.1523/JNEUROSCI.0977-15.2015

1581 Davis, B.L., MacNeilage, P., \& Matyear, C.L. (2002). Acquisition of Serial Complexity in Speech 1582 Production: A Comparison of Phonetic and Phonological Approaches to First Word Production. Phonetica, 59, 75-107. http://dx.doi.org/10.1159/000066065

1584 Dayan, P., \& Abbott, L. (2001). Theoretical Neuroscience. The MIT Press, Cambridge, MA. 
THE ROLE OF MOTOR INFORMATION IN SPEECH PERCEPTION

1585 DeCasper, A.J., \& Fifer, W.P. (1980). Of human bonding: newborns prefer their mother's voice. $1586 \quad$ Science, 208, 1174-1176.

1587 Dehaene-Lambertz, G., Dehaene, S., \& Hertz-Pannier, L. (2002). Functional Neuroimaging of Speech Perception in Infants. Science, 298, 2013-2015. http://dx.doi.org/10.1126/science.1077066

1590 Dehaene-Lambertz, G., Hertz-Pannier, L., Dubois, J., Mériaux, S., Roche, A., Sigman, M., \& Dehaene, S. (2006). Functional organization of perisylvian activation during presentation of sentences in preverbal infants. Proceedings of the National Academy of Sciences, 103, 14240-

1594 Delattre, P. C., Liberman, A. M. \& Cooper, F. S. (1955) Acoustic loci and transitional cues for consonants. Journal of the Acoustical Society of America, 27, 769-73. http://dx.doi.org/10.1121/1.1908024

Deng, L. (1999). Computational models for auditory speech processing,. In K. Ponting (Ed.) Computational Models for Speech Pattern Processing, pp. 67-77. NewYork: Springer-Verlag, NATO ASI.

Deng, L. \& Ma, J. (2000). Spontaneous speech recognition using a statistical coarticulatory model for the vocal- tract-resonance dynamics. The Journal of the Acoustical Society of America, 108, 3036-3048. http://dx.doi.org/10.1121/1.1315288

Deng, L., Ramsay, G., \& Sun, D. (1997). Production models as a structural basis for automatic speech recognition. Speech Communication, 22, 93-111. http://dx.doi.org/10.1016/S01676393(97)00018-6

Diehl, R., Lotto, A., \& Holt, L. (2004). Speech perception. Annual Review of Psychology, 55, 149179. http://dx.doi.org/10.1146/annurev.psych.55.090902.142028

1608 Dillon, B., Dunbar, E., \& Idsardi, W. (2013). A single-stage approach to learning phonological categories: Insights from Inuktitut. Cognitive Science, 37, 344-377. 

http://dx.doi.org/10.1111//ogs.12008

1611 Dole, M., Loevenbruck, H., Pascalis, O., Schwartz, J.-L., \& Vilain, A. (2015). Perceptual abilities in relation with motor development in the first year of life. WILD 2015 International Conference, Stockholm.

1614 Dole, M., Loevenbruck, H., Pascalis, O., Schwartz, J.L, Vilain, A. (2016). Phoneme categorization depends on production abilities during the first year of life. XX ICIS 2016 - The International Congress on Infant Studies.

Eimas, P. D. (1999). Segmental and syllabic representations in the perception of speech by young infants. The Journal of the Acoustical Society of America, 105,1901-1911. http://dx.doi.org/10.1121/1.426726 Science, 171, 303-306. http://dx.doi.org/10.1126/science.171.3968.303

Ernst, M. O., \& Banks, M. S. (2002). Humans integrate visual and haptic information in a statistically optimal fashion. Nature, 415, 429-433. http://dx.doi.org/10.1038/415429a

1624 Fadiga, L., Craighero, L., Buccino, G., \& Rizzolatti, G. (2002). Speech listening specifically modulates the excitability of tongue muscles: A TMS study. European Journal of Neuroscience, 15, 399-402. http://dx.doi.org/10.1046/j.0953-816x.2001.01874.x

Feldman, N. H., \& Griffiths, T. L. (2007). A rational account of the perceptual magnet effect. In Proceedings of the 29th Annual Conference of the Cognitive Science Society, pp. 257-262.

Feldman, N. H., Griffiths, T. L., \& Morgan, J. L. (2009a). The influence of categories on perception: Explaining the perceptual magnet effect as optimal statistical inference. Psychological Review,

1632 Feldman, N. H., Griffiths, T. L., \& Morgan, J. L. (2009b). Learning phonetic categories by learning a 1633 lexicon. In Proceedings of the 31st Annual Conference of the Cognitive Science Society, pp. $2208-2213$. 
1635 Feldman, N. H., Griffiths, T. L., Goldwater, S., \& Morgan, J. L. (2013). A role for the developing

1636

1637

1638

1639

1640

1641

1642

1643

1644

1645

1646

1647

1648

1649

1650

1651

1652

1653

1654

1655

1656

1657

1658

1659 lexicon in phonetic category acquisition. Psychological Review, 120, 751-778. http://dx.doi.org/10.1037/a0034245

Formisano, E., De, M.F., Bonte, M., \& Goebel, R. (2008). "Who" is saying "what"? brain-based decoding of human voice and speech. Science, 322, 970-973. http://dx.doi.org/10.1126/science.1164318

Fowler, C., \& Dekle, D. (1991). Listening with eye and hand: crossmodal contributions to speech perception. Journal of Experimental Psychology: Human Perception and Performance, 17, 816-828. http://dx.doi.org/doi: 10.1037/0096-1523.17.3.816

Frankel, J., Richmond, K., King, S., et Taylor, P. (2000). An automatic speech recognition system using neural networks and linear dynamic models to recover and model articulatory traces. In Proc. Sixth International Conference on Spoken Language Processing, Vol. 4. International Speech Communication Association.

Galantucci, B., Fowler, C.A., \& Turvey, M.T. (2006). The motor theory of speech perception reviewed. Psychonomic Bulletin \& Review, 13, 361-377. http://dx.doi.org/10.3758/BF03193857

Gaskell, M. G., \& Marslen-Wilson, W. D. (1997). Integrating form and meaning: A distributed model of speech perception. Language and Cognitive Processes, 12, 613-656.

Gilet, E., Diard, J., \& Bessière, P. (2011). Bayesian action-perception computational model: interaction of production and recognition of cursive letters. PLOS ONE, 6, e20387. http://dx.doi.org/10.1371/journal.pone.0020387

Goldstein, L., \& Fowler, C. A. (2003). Articulatory phonology: A phonology for public language use. In N. O. Schiller \& A. Meyer (Eds.), Phonetics and phonology in language comprehension and production: Differences and similarities (pp. 159-207). Berlin: Mouton de Gruyter.

Grabski, K., Tremblay, P., Gracco, V.L., Girin, L., \& Sato, M. (2013). A mediating role of the 
1660

1661

1662

1663

1664

1665

1666

1667

1668

1669

1670

1671

1672

1673

1674

1675

1676

1677

1678

1679

1680

1681

1682

1683

1684

auditory dorsal pathway in selective adaptation to speech: A state-dependent transcranial magnetic stimulation study. Brain Research, 1515, 55-65.

http://dx.doi.org/10.1016/j.brainres.2013.03.024

Grafton, S.T., Arbib M.A., Fadiga, L., \& Rizzolatti, G. (1996). Localization of grasp representations in humans by positron emission tomography. 2. Observation compared with imagination. Experimental Brain Research, 112, 103-11. http://dx.doi.org/10.1007/BF00227183

Green, D. M. \& Swets, J. A. (1966). Signal detection theory and psychophysics. John Wiley \& Sons, Ltd, New York, NY, USA.

Guenther, F. H. (2006). Cortical interactions underlying the production of speech sounds. Journal of Communication Disorders, 39, 350-365. http://dx.doi.org/10.1016/j.jcomdis.2006.06.013

Guenther, F. H., Ghosh, S. S., \& Tourville, J. A. (2006). Neural modeling and imaging of the cortical interactions underlying syllable production. Brain and Language, 96, 280-301. http://dx.doi.org/10.1016/j.band1.2005.06.001

Guenther, F. H., Hampson, M., \& Johnson, D. (1998). A theoretical investigation of reference frames for the planning of speech movements. Psychological Review, 105, 611-633. http://dx.doi.org/10.1037/0033-295X.105.4.611-633

Halle, M., \& Stevens, K. N. (1959). Analysis by synthesis. In W. Wathen-Dunn \& L. E. Woods (Eds.), Proceedings of the seminar on speech compression and processing. USAF Camb. Res. Ctr. 2: Paper D7.

Hermansky, H. (1998). Should recognizers have ears? Speech Communication, 25, 3-27.

Hickok, G. (2012). Computational neuroanatomy of speech production. Nature Reviews Neuroscience. 13, 135-145. doi:10.1038/nrn3158

Hinton, G., Deng, L., Yu, D., Dahl, G., Mohamed, A., Jaitly, N., A. Senior, Vanhoucke, V., Nguyen, P., Sainath, T., \& Kingsbury, B. (2012). Deep neural networks for acoustic modeling in speech recognition: The shared views of four research groups. IEEE Signal Process. Magazine, 29, 
$82-97$.

1686

Hochmann, J.R., \& Papeo, L. (2014). The Invariance Problem in Infancy: A Pupillometry Study. Psychological Science, 25, 2038-2046. http://dx.doi.org/10.1177/0956797614547918

Houde, J. F., \& Nagarajan, S. S. (2011). Speech production as state feedback control. Frontiers in Human Neuroscience, 5. doi:10.3389/fnhum.2011.00082

Huang, X., \& Deng, L. (2010). An Overview of Modern Speech Recognition., in R. Herbrich \& T. Graepel (Eds.) Handbook of Natural Language Processing, Second Edition, pp. 339-366. Chapman \& Hall/CRC.

Iacoboni, M., Woods, R.P., Brass, M., Bekkering, H., Mazziotta, J.C., \& Rizzolatti, G. (1999). Cortical Mechanisms of Human Imitation. Science, 286, 2526-2528. http://dx.doi.org/10.1126/science.286.5449.2526.

Imada T, Zhang, Y., Cheour, M., Taulu, S., Ahonen, A., \& Kuhl, P.K. (2006) Infant speech perception activates Broca's area: A developmental magnetoencephalography study. NeuroReport, 17, 957-962.

Ito, T., Tiede, M., \& Ostry, D.J. (2009). Somatosensory function in speech perception. Proceedings of the National Academy of Sciences, 106, 1245-1248. http://dx.doi.org/10.1073/pnas.0810063106.

Jacquemot, C., Dupoux, E., \& Bachoud-Lévi, A.-C. (2007). Breaking the mirror: asymmetrical disconnection between the phonological input and output codes. Cognitive Neuropsychology, 24, 3-22. http://dx.doi.org/10.1080/02643290600683342

Jones, J.A., \& Callan, D.E. (2003). Brain activity during audiovisual speech perception: An fMRI study of the McGurk effect. NeuroReport, 14, 1129-1133. http://dx.doi.org/10.1097/00001756-200306110-00006

Jusczyk, P. W., \& Derrah, C. (1987). Representation of speech sounds by young infants. Developmental Psychology, 23, 648-654. http://dx.doi.org/10.1037/0012-1649.23.5.648 
1710 Kewley-Port, D. (1983). Time-varying features as correlates of place of articulation in stop

1711

1712

1713

1714

1715

1716

1717

1718

1719

1720

1721

1722

1723

1724

1725

1726

1727

1728

1729

1730

1731

1732

1733

1734 consonants. Journal of the Acoustical Society of America, 73, 322-335. http://dx.doi.org/10.1121/1.388813

Kiebel, S. J., Daunizeau, J., \& Friston, K. J. (2009). Perception and hierarchical dynamics. Frontiers in Neuroinformatics, 3, 20. http://dx.doi.org/10.3389/neuro.11.020.2009

Kingston, J., \& Diehl, R.L. (1994). Phonetic knowledge. Language, 70, 419-54. http://dx.doi.org/10.1353/lan.1994.0023.

Kleinschmidt, D., \& Jaeger, T. F. (2011). A Bayesian belief updating model of phonetic recalibration and selective adaptation. In Proceedings of the 2nd Workshop on Cognitive Modeling and Computational Linguistics, pages 10-19. Association for Computational Linguistics.

Kleinschmidt, D. \& Jaeger, T. F. (2015). Robust speech perception: Recognizing the familiar, generalizing to the similar, and adapting to the novel. Psychological Review, 122, 148-203. http://dx.doi.org/10.1037/a0038695

Kluender KR. (1994). Speech perception as a tractable problem in cognitive science. In M.A. Gernsbacher (Ed.) Handbook of Psycholinguistics (pp. 173-217). San Diego, CA: Academic.

Kröger, B.J., Kannampuzha, J., \& Kaufmann, E. (2014). Associative learning and self-organization as basic principles for simulating speech acquisition, speech production, and speech perception. EPJ Nonlinear Biomedical Physics, 2. http://dx.doi.org/10.1140/epjnbp15

Kröger, B.J., Kannampuzha, J., \& Neuschaefer-Rube, C. (2009). Towards a neurocomputational model of speech production and perception. Speech Communication, 51, 793-809. http://dx.doi.org/10.1016/j.specom.2008.08.002

Kuhl, P. K. (1979). Speech perception in early infancy: Perceptual constancy for spectrally dissimilar vowel categories. Journal of the Acoustical Society of America, 66, 1668-1679.

Kuhl, P. K. (1983). Perception of auditory equivalence classes for speech in early infancy. Infant Behavior \& Development, 6, 263-285. 
1735 Kuhl, P. K. (2004). Early language acquisition: cracking the speech code. Nature Reviews

1736

1737

1738

1739

1740

1741

1742

1743

1744

1745

1746

1747

1748

1749

1750

1751

1752

1753

1754

1755

1756

1757

1758

1759 Neuroscience, 5, 831-843. http://dx.doi.org/10.1038/nrn1533

Kuhl, P.K., Conboy, B.T., Coffey-Corina, S., Padden, T., Rivera-Gaxiola, M., \& Nelson, T. (2008). Phonetic learning as a pathway to language: New data and native language magnet theory expanded (NLM-e). Philosophical Transactions of the Royal Society B: Biological Sciences, 363, 979-1000. http://dx.doi.org/10.1098/rstb.2007.2154

Kuhl, P. K., \& Meltzoff (1996). A. Infant vocalizations in response to speech: vocal imitation and developmental change. Journal of the Acoustical Society of America. 100, 2425-2438.

Kuhl, P.K., Ramírez, R.R., Bosseler, A., Lotus Lin, J.F., \& Imada, T. (2014). Infants' brain responses to speech suggest Analysis by Synthesis. Proceedings of the National Academy of Sciences, 111, 12572-12573. http://dx.doi.org/10.1073/pnas.1410963111

Kuhl, P. K., Williams, K. A., Lacerda, F., Stevens, K. N. \& Lindblom, B. (1992). Linguistic experience alters phonetic perception in infants by 6 months of age. Science, 255, 606-608. http://dx.doi.org/10.1126/science.1736364

Laurent, R., Schwartz, J.-L., Bessière, P., \& Diard, J. (2013). A computational model of perceptuomotor processing in speech perception: learning to imitate and categorize synthetic $\mathrm{CV}$ syllables. In Proceedings of InterSpeech (pp. 2797-2801). Lyon, France.

Lebeltel, O., Bessière, P., Diard, J., \& Mazer, E. (2004). Bayesian robot programming. Autonomous Robots, 16, 49-79. http://dx.doi.org/10.1023/B:AURO.0000008671.38949.43

Liberman, A. M., Cooper, F. S., Shankweiler, D. P., \& Studdert-Kennedy, M. (1967). Perception of speech code. Psychological Review, 74, 431-461. http://dx.doi.org/10.1037/h0020279

Liberman, A.M., \& Mattingly, I. (1985). The motor theory of speech perception revised. Cognition, 21, 1-36. http://dx.doi.org/10.1016/0010-0277(85)90021-6

Liberman, A.M., \& Mattingly, I.G. (1989). A specialization for speech perception. Science, 243, 489494. http://dx.doi.org/10.1126/science. 2643163 
1760 Liberman, A.M., \& Whalen, D.H. (2000). On the relation of speech to language. Trends in Cognitive Sciences, 4, 187-196. http://dx.doi.org/10.1016/S1364-6613(00)01471-6

1762

1763

1764

1765

1766

1767

1768

1769

1770

1771

1772

1773

1774

1775

1776

1777

1778

1779

1780

1781

1782

1783

1784

Lotto, A.J. (2000). Language acquisition as complex category formation. Phonetica, 57, 189-96. http://dx.doi.org/10.1159/000028472

Luce, P. A., Goldinger, S. D., Auer, E. T., \& Vitevitch, M. S. (2000). Phonetic priming, neighborhood activation, and PARSYN. Perception and Psychophysics, 62, 615-625.

MacNeilage, P.F. (1998). The frame/content theory of evolution of speech production. Behavioral and Brain Sciences, 21, 499-546. http://dx.doi.org/10.1017/S0140525X98001265

Maeda, S. (1990). Compensatory articulation during speech: Evidence from the analysis and synthesis of vocal tract shapes using an articulatory model. In W. Hardcastle \& A. Marchal (Eds.), Speech production and speech modeling (pp. 131-149). Kluwer Academic.

Maeda, S. \& Honda, K. (1994). From EMG to formant patterns: the implication of vowel spaces. Phonetica, 51, 17-29. http://dx.doi.org/10.1159/000261955

Marr, D. (1982). Vision. A Computational Investigation into the Human Representation and Processing of Visual Information. W.H. Freeman and Company, New York, USA.

Massaro, D. W. (1987). Speech Perception by Ear and Eye: A Paradigm for Psychological Inquiry. Laurence Erlbaum Associates, London.

Massaro, D. W. (1998). Perceiving Talking Faces. MIT, Cambridge, Ma).

Massaro, D.W., \& Oden, G.C. (1980). Evaluation and integration of acoustic features in speech perception. Journal of the Acoustical Society of America, 67, 996-1013. http://dx.doi.org/10.1121/1.383941

McClelland, J. L., \& Elman, J. L. (1986). The TRACE model of speech perception. Cognitive Psychology, 18, 1-86. http://dx.doi.org/10.1016/0010-0285(86)90015-0

McGurk, H., \& MacDonald, J. (1976). Hearing lips and seeing voices. Nature, 264, 746-748. http://dx.doi.org/doi:10.1038/264746a0 
1785 McMurray, B., Aslin, R. N., \& Toscano, J. C. (2009). Statistical learning of phonetic categories:

1786

1787

1788

1789

1790

1791

1792

1793

1794

1795

1796

1797

1798

1799

1800

1801

1802

1803

1804

1805

1806

1807

1808

1809 insights from a computational approach. Developmental science, 12, 369-378. http://dx.doi.org/10.1111/j.1467-7687.2009.00822.x

Mehler, J., Jusczyk, P., Lambertz, G., Halsted, N., Bertoncini, J., \& Amiel-Tison, C. (1988). A precursor of language acquisition in young infants. Cognition, 29, 143-178.

Meister, I.G., Wilson, S.M., Deblieck, C., Wu, A.D., \& Iacoboni, M. (2007). The essential role of premotor cortex in speech perception. Current Biology, 17, 1692-1696. http://dx.doi.org/10.1016/j.cub.2007.08.064

Meltzoff, A.N., \& Moore, M.K. (1977). Imitation of facial and manual gestures by human neonates. Science, 4312, 75-78. http://dx.doi.org/10.1126/science.198.4312.75

Ménard, L., Schwartz, J.-L., Boë, L.-J., Kandel, S., \& Vallée, N. (2002). Auditory normalization: of French vowels synthesized by an articulatory model simulating growth from birth to adulthood. Journal of the Acoustical Society of America, 111, 1892-1905. http://dx.doi.org/10.1121/1.1459467

Ménard, L., Schwartz, J.-L., \& Aubin, J. (2008). Invariance and variability in the production of the height feature in French vowels. Speech Communication, 50, 14-28. http://dx.doi.org/10.1016/j.specom.2007.06.004

Ménard, L., Schwartz, J.-L., \& Boë, L.-J. (2004). The role of vocal tract morphology in speech development: Perceptual targets and sensori-motor maps for French synthesized vowels from birth to adulthood. Journal of Speech Language and Hearing Research, 47, 1059-1080. http://dx.doi.org/10.1044/1092-4388(2004/079)

Meunier, C. (2007). Phonétique acoustique. In P. Auzou (Ed.), Les dysarthries (pp. 164-173). Solal.

Moore, R. (2007). Spoken language processing: Piecing together the puzzle. Speech Communication, 49, 418-435. http://dx.doi.org/10.1016/j.specom.2007.01.011

Möttönen, R., Dutton, R., \& Watkins, K.E. (2013). Auditory-motor processing of speech sounds. 
1810

1811

1812

1813

1814

1815

1816

1817

1818

1819

1820

1821

1822

1823

1824

1825

1826

1827

1828

1829

1830

1831

1832

1833

1834

Cerebral Cortex, 23, 1190-1197. http://dx.doi.org/10.1093/cercor/bhs110

Möttönen, R., \& Watkins, K.E. (2009). Motor representations of articulators contribute to categorical perception of speech sounds. The Journal of Neuroscience, 29, 9819-9825. http://dx.doi.org/10.1523/JNEUROSCI.6018-08.2009

Moulin-Frier, C., Diard, J., Schwartz, J.-L., \& Bessière, P. (2015). COSMO (“Communicating about Objects using Sensory-Motor Operations"): a Bayesian modeling framework for studying speech communication and the emergence of phonological systems. Journal of Phonetics, 53, 5-41.

Moulin-Frier, C., Laurent, R., Bessière, P., Schwartz, J.-L, \& Diard, J. (2012). Adverse conditions improve distinguishability of auditory, motor, and perceptuo-motor theories of speech perception: An exploratory Bayesian modelling study. Language and Cognitive Processes, 27, 1240-1263. http://dx.doi.org/10.1080/01690965.2011.645313

Moulin-Frier, C., Schwartz, J.-L., Diard, J., \& Bessière, P. (2011). Emergence of phonology through deictic games within a society of sensori-motor agents in interaction. In A. Vilain, J.-L. Schwartz, C. Abry \& J. Vauclair (Eds.) Primate Communication and Human Language (pp. 193-220). John Benjamins Publishing Company.

Nearey, T.M. (1990). The segment as a unit of speech perception. Journal of Phonetics, 18, 347-73

Norris D., \& McQueen J.M. (2008). Shortlist B: a Bayesian model of continuous speech recognition. Psychol Rev., 115, 357-95. doi: 10.1037/0033-295X.115.2.357.

Öhman, S. (1966). Coarticulation in VCV utterances: spectrographic measurements. Journal of the Acoustical Society of America, 39, 151-168. http://dx.doi.org/10.1121/1.1909864

Ojanen, V., Möttönen, R., Pekkola, Jääskeläinen, I., Joensuu, R., Autti, T., \& Sams, M. (2005). Processing of audiovisual speech in Broca's area. NeuroImage, 25, 333-338. http://dx.doi.org/10.1016/j.neuroimage.2004.12.001.

Pasley, B.N., David, S.V., Mesgarani, N., Flinker, A., Shamma, S.A., Crone, N. E. et al. (2012). 
Reconstructing speech from human auditory cortex. PLoS Biol. 10:e1001251. doi:10.1371/journal.pbio.1001251

Patri, J.F. Diard, J., \& Perrier, P. (2015). Optimal speech motor control and token-to-token variability: a Bayesian modeling approach. Biological Cybernetics, 109, 611-626. http://link.springer.com/article/10.1007/s00422-015-0664-4.

Perkell, J. \& Klatt, D. H. (1986). Invariance and variability in speech processes. Erlbaum.

Perrier, P. (2005). Control and representations in speech production. ZAS Papers in Linguistics, 40, $109-132$.

Perrier, P., Lœvenbruck, H., \& Payan, Y. (1996). Control of tongue movements in speech: The equilibrium point hypothesis perspective. Journal of Phonetics, 24, 53-75.

Pitermann, M. (2000). Effect of speaking rate and contrastive stress on formant dynamics and vowel perception. Journal of the Acoustical Society of America, 107, 3425-3437. http://dx.doi.org/10.1121/1.429413

Polka, L., Masapollo, M., \& Ménard, L. (2014). Who’s talking now? Infants’ perception of vowels with infant vocal properties. Psychological Science, 25, 1448-1456.

Pulvermüller, F., Huss, M., Kherif, F., Moscoso del Prado Martin, F., Hauk, O., \& Shtyrov, Y. (2006). Motor cortex maps articulatory features of speech sounds. Proceedings of the National Academy of Sciences, 103, 7865-7870. http://dx.doi.org/10.1073/pnas.0509989103

Rizzolatti, G., Fadiga, L., Gallese, V., \& Fogassi, L. (1996a). Premotor cortex and the recognition of motor actions. Cognitive Brain Research, 3, 131-141. http://dx.doi.org/10.1016/09266410(95)00038-0

Rizzolatti, G., Fadiga, L., Matelli, M., Bettinardi, V., Paulesu, E., Perani, D., \& Fazio, F. (1996b). Localization of grasp representations in humans by PET: 1. observation vs. execution. Experimental Brain Research, 111, 246-252. http://dx.doi.org/10.1007/BF00227301 
1860

1861

1862

1863

1864

1865

1866

1867

1868

1869

1870

1871

1872

1873

1874

1875

1876

1877

1878

1879

1880

1881

1882

1883

1884

in bimodal speech : auditory, visual, and audio-visual identification of French oral vowels in noise. Journal of the Acoustical Society of America, 103, 3677-3689. http://dx.doi.org/10.1121/1.423069

Rogers, J. C., Möttönen, R., Boyles, R., \& Watkins, K. E. (2014). Discrimination of speech and nonspeech sounds following theta-burst stimulation of the motor cortex. Frontiers in Psychology, 5, 754. http://dx.doi.org/10.3389/fpsyg.2014.00754

Rouder, J. N., \& Lu, J. (2005). An introduction to Bayesian hierarchical models with an application in the theory of signal detection. Psychonomic Bulletin \& Review, 12, 573-604. http://dx.doi.org/10.3758/BF03196750

Sato, M., Grabski, K., Glenberg, A., Brisebois, A., Basirat, A., Ménard, L. \& Cattaneo, L. (2011). Articulatory bias in speech categorization: evidence from use-induced motor plasticity. Cortex, 47, 1001-1003. http://dx.doi.org/10.1016/j.cortex.2011.03.009

Sato, M., Tremblay, P. \& Gracco, V. (2009). A mediating role of the premotor cortex in phoneme segmentation. Brain and Language, 111, 1-7. http://dx.doi.org/10.1016/j.bandl.2009.03.002

Savariaux, C., Perrier P., \& Orliaguet, J.-P. (1995). Compensation strategies for the perturbation of the rounded vowel $[\mathrm{u}]$ using a lip-tube: a study of the control space in speech production. Journal of the Acoustical Society of America, 98, 2428-2442. http://dx.doi.org/10.1121/1.413277

Savariaux, C., Perrier, P., Orliaguet, J.P. \& Schwartz, J.-L. (1999). Compensation strategies for the perturbation of French [u] using a lip tube. II. Perceptual analysis. Journal of the Acoustical Society of America, 106, 381-393. http://dx.doi.org/10.1121/1.427063

Scharenborg, O., Norris, D., Ten Bosch, L., \& McQueen, J. M. (2005). How should a speech recognizer work? Cognitive Science, 29, 867-918. doi:10.1207/s15516709cog0000_37.

Schwartz, J.L. (2010). A reanalysis of McGurk data suggests that audiovisual fusion in speech perception is subject-dependent. Journal of the Acoustical Society of America, 127, 1584- 

1594. http://dx.doi.org/10.1121/1.3293001

Schwartz, J.-L., Abry, C., Boë, L.-J., \& Cathiard, M. (2002). Phonology in a theory of perception-foraction-control. In J. Durand, B. Laks (Eds.) Phonology: from Phonetics to Cognition (pp. 255280). Oxford: Oxford University Press.

Schwartz, J.-L., Basirat, A., Ménard, L., \& Sato, M. (2012a). The Perception-for-Action-Control Theory (PACT): A perceptuo-motor theory of speech perception. Journal of Neurolinguistics, 25, 336-354. doi:10.1016/j.jneuroling.2009.12.004

Schwartz, J.-L., Boë, L.-J., \& Abry, C. (2007). Linking the Dispersion-Focalization Theory (DFT) and the Maximum Utilization of the Available Distinctive Features (MUAF) principle in a Perception-for-Action-Control Theory (PACT). In M.J. Solé, P. Beddor \& M. Ohala (Eds.) Experimental Approaches to Phonology (pp. 104-124). Oxford University Press.

Schwartz J.-L., Boë L.-J., Badin P., \& Sawallis R. T. (2012b). Grounding stop place systems in the perceptuo-motor substance of speech: On the universality of the labial-coronal-velar stop series, Journal of Phonetics, 40, 20-36.

Schwartz, J.L., Boë, L.J., Vallée, N., \& Abry , C. (1997). Major trends in vowel system inventories. Journal of Phonetics, 25, 233-254.

Shiller, D.M., Sato, M., Gracco, V.L., \& Baum, S.R. (2009). Perceptual recalibration of speech sounds following speech motor learning. Journal of the Acoustical Society of America, 125, 1103-1113. http://dx.doi.org/10.1121/1.3058638

Skipper, J.I., Devlin, J.T., \& Lametti, D.R. (2017). The hearing ear is always found close to the speaking tongue: Review of the role of the motor system in speech perception. Brain and Language, 164, 77-105. http://dx.doi.org/10.1016/j.bandl.2016.10.004

Skipper, J.I., van Wassenhove, V., Nusbaum, H.C. \& Small, S.L. (2007). Hearing lips and seeing voices: how cortical areas supporting speech production mediate audiovisual speech perception. Cerebral Cortex, 17, 2387-2399. http://dx.doi.org/10.1093/cercor/bhl147. 
1910 Sonderegger, M., \& Yu, A. (2010). A rational account of perceptual compensation for coarticulation. In Proceedings of the 32nd Annual Meeting of the Cognitive Science Society, pp. 375-380.

Stevens, K.N. (1972). The quantal nature of speech: evidence from articulatory-acoustic data. In E.

Stevens, K.N. (1980). Acoustic correlates of some phonetic categories. Journal of the Acoustical Society of America, 68, 836-842. http://dx.doi.org/10.1121/1.384823

1916

Stevens, K.N. (1989). On the quantal nature of speech. Journal of Phonetics, 17, 3-45.

Stevens, K.N., Blumstein, S.E. (1978). Invariant cues for place of articulation in stop consonants. Journal of the Acoustical Society of America, 64, 1358-1368. http://dx.doi.org/10.1121/1.382102

Stevens, K. N., \& Halle, M. (1967). Remarks on analysis by synthesis and distinctive features. In W. Wathen-Dunn (Ed.), Models for the perception of speech and visual form (pp. 88-102). Cambridge, MA: MIT Press.

Strange, W. (1989). Evolving theories of vowel perception. Journal of the Acoustical Society of America, 85, 2081-2087. http://dx.doi.org/10.1121/1.397860

Sumby, W.H., \& Pollack, I. (1954). Visual contribution to speech intelligibility in noise. Journal of the Acoustical Society of America, 26, 212-215. http://dx.doi.org/doi: 10.1121/1.1907309

Summerfield, Q. (1987). Some preliminaries to a comprehensive account of audio-visual speech perception. In B Dodd and R Campbell (Eds.) Hearing by eye: the psychology of lip-reading (pp 3-51). Lawrence Erlbaum Associates, London.

Sun, J., \& Deng, L. (2002). An overlapping-feature based phonological model incorporating linguistic constraints: Applications to speech recognition. Journal of the Acoustical Society of America, $111,1086-1101$.

Sussman, H., Fruchter, D., Hilbert, J., \& Sirosh, J. (1998). Linear correlates in the speech signal: the orderly output constraint. Behavioral and Brain Sciences, 21(02), 241-259. 
Sussman, H. M., Hoemeke, K. \& Ahmed, F. (1993) A cross-linguistic investigation of locus equations as a relationally invariant descriptor for place of articulation. Journal of the Acoustical Society of America, 94, 1256-68. http://dx.doi.org/10.1121/1.408178

Sussman, H. M., McCaffrey, H. A. \& Matthews, S. A. (1991) An investigation of locus equations as a source of relational invariance for stop place categorization. Journal of the Acoustical Society of America, 90, 1309-25. http://dx.doi.org/10.1121/1.401923

Tanner, W. P., \& Swets, J. A. (1954). A decision-making theory of visual detection. Psychological Review, 61, 401-409. http://dx.doi.org/10.1037/h0058700

Toscano, J. C., \& McMurray, B. (2008). Using the distributional statistics of speech sounds for weighting and integrating acoustic cues. In Proceedings of the 30th Annual Conference of the Cognitive Science Society, pp. 433-439.

Toscano, J. C., \& McMurray, B. (2010). Cue integration with categories: Weighting acoustic cues in speech using unsupervised learning and distributional statistics. Cognitive Science, 34, 434464. http://dx.doi.org/10.1111/j.1551-6709.2009.01077.x

Treille, A., Cordeboeuf, C., Vilain, C., \& Sato, M. (2014). Haptic and visual information speed up the neural processing of auditory speech in live dyadic interactions. Neuropsychologia, 57, 71-77. http://dx.doi.org/doi:10.1016/j.neuropsychologia.2014.02.004

Vallabha, G. K., McClelland, J. L., Pons F., Werker, J. F., \& Amano, S. (2007). Unsupervised learning of vowel categories from infant-directed speech. Proceedings of the National Academy of Sciences, 104, 13273-13278. http://dx.doi.org/10.1073/pnas.0705369104

Watkins, K.E., Strafella, A.P., \& Paus, T. (2003). Seeing and hearing speech excites the motor system involved in speech production. Neuropsychologia, 41, 989-994. http://dx.doi.org/10.1016/S0028-3932(02)00316-0

Werker, J.F., \& Hensch, T.K. (2015). Critical Periods in Speech Perception: New Directions. Annu. Rev. Psychol.. 66, 173-96. 
1960 Werker, J.F., \& Tees, R.C. (1984). Cross-language speech perception: Evidence for perceptual reorganization during the first year of life. Infant Behavior and Development, 7, 49-63. http://dx.doi.org/10.1016/S0163-6383(84)80022-3

Wilson, S. M., \& Iacoboni, M. (2006). Neural responses to non-native phonemes varying in productibility: Evidence for the sensorimotor nature of speech perception. NeuroImage, 33, 316-325. http://dx.doi.org/10.1016/j.neuroimage.

Wilson, S. M., Saygin, A. P., Sereno, M. I., \& Iacoboni, M. (2004). Listening to speech activates motor areas involved in speech production. Nature Neuroscience, 7, 701-702. http://dx.doi.org/10.1038/nn1263

Zekveld, A.A., Heslenfeld, D.J., Festen, J.M., \& Schoonhoven, R. (2006). Top-down and bottom-up processes in speech comprehension. NeuroImage, 32, 1826-1836. http://dx.doi.org/10.1016/j.neuroimage.2006.04.199 
Footnotes

${ }^{(1)}$ It is well known that other sensory systems may intervene in speech perception: such as vision, through lip-reading (Sumby \& Pollack, 1954; McGurk \& MacDonald, 1976; Summerfield, 1987) but also possibly somato-sensory processing (Fowler \& Dekle, 1991; Ito, Tiede \& Ostry, 2009; Treille et al., 2014). However, in this paper we will focus on the

1980 auditory vs. motor systems, acknowledging that these other sensory systems could be 1981 incorporated as additional sensory inputs inside a perceptuo-motor framework.

${ }^{(2)}$ Here, we use the term "communication object" in a broad sense, conflating

1983

1984 different levels of analysis (phonetics, phonology, syntax, semantics). In this paper, objects will only refer to phonological entities.

(3) Technically, probability values below the $\varepsilon$ threshold are set to $\varepsilon$ during perception inference, but are set to 0 during learning. This makes learning approximate but fast, as portions of spaces with very low probabilities are dismissed altogether.

${ }^{(4)}$ Notice that the visual system could intervene in this process, especially considering the natural complementarity of auditory and visual representations in the depiction of vowels and plosives (Summerfield, 1987; Robert-Ribes et al., 1998).

${ }^{(5)}$ VLAM is actually an articulatory rather than a motor model of speech production. VLAM inputs are parameters controlling the shape of the tongue and lips and the position of the jaw, which are themselves the results of motor commands at a higher level (see e.g. Perrier et al., 1996; Perrier, 2005). We consider as a simplification that VLAM articulatory parameters are part of the control system and hence could provide "motor commands" at a certain level of representation in the motor pathway.

${ }^{(6)}$ While we display mean entropies in this Figure, averaging over the 9 syllables, there are actually differences between entropy dynamics among the different syllables, particularly in the motor space. This is clearly seen in Figure 14, where it appears that 
2000 convergence is more rapid for $/ \mathrm{u} /$ than for the other two vowels. The likely reason is that the

2001 available articulatory space for achieving the adequate formants is more restricted for $/ \mathrm{u} /$ in 2002 the available 3-D articulatory space in VLAM. Notice that slower articulatory convergence 2003 (displayed in Figure 14) can occur in spite of rapid acoustic convergence (as displayed by the 2004 rapid formant convergence for / $\mathbf{i} /$ in Figure 14). It is beyond the scope of the present paper to 2005 discuss the importance and significance of these differences in convergence among vowels, 2006 plosives or syllables. 


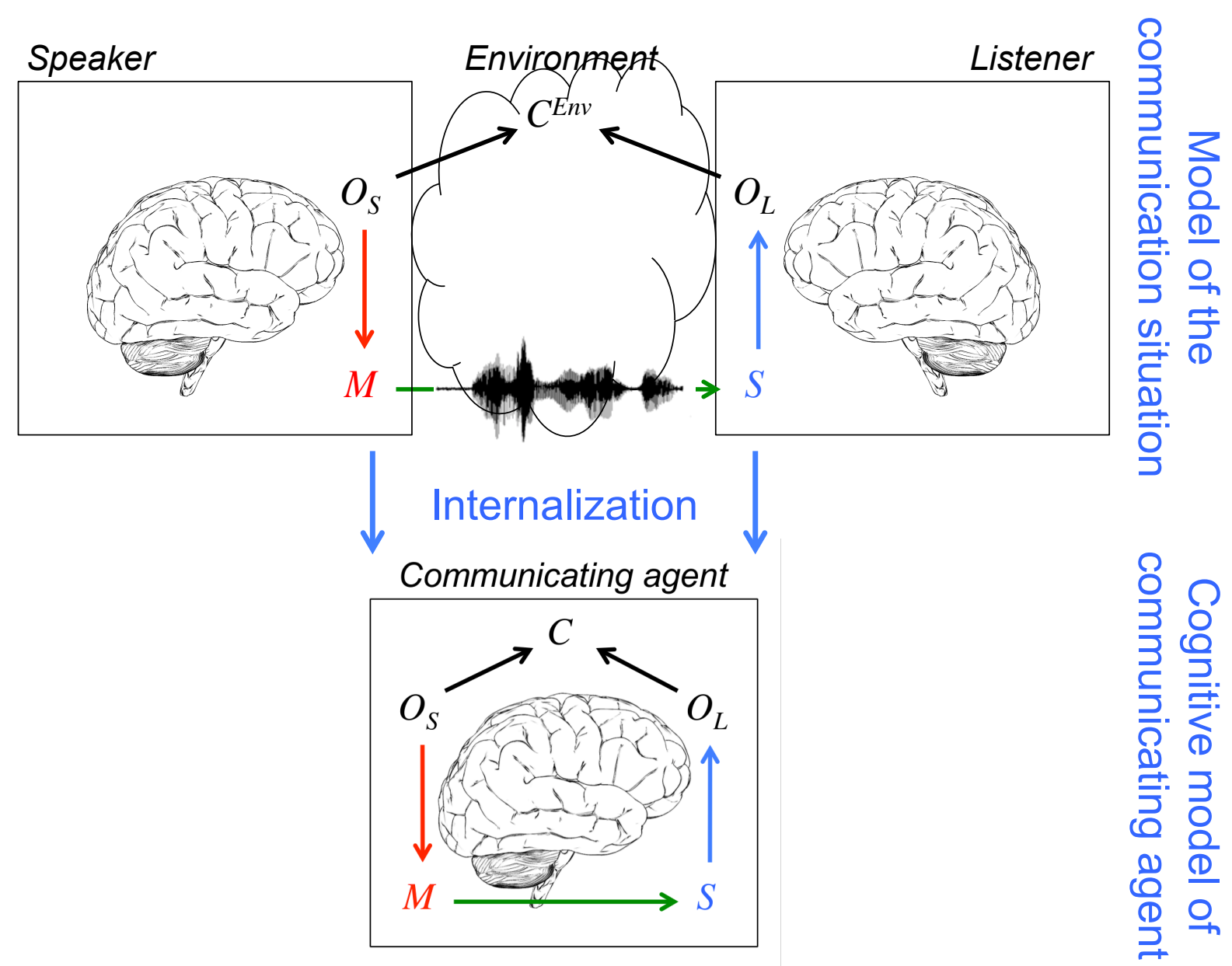

2009 Figure 1. The communication situation, which involves a speaker agent and listener agents

2010 interacting within an environment, is internalized in communicating agents. Top, model of the

2011 communication situation: the speaker wants to mention a linguistic object (in a broad sense,

2012 see footnote 2) $\mathrm{O}_{\mathrm{S}}$. She/he produces a motor gesture M leading to the production of a sound S

2013 propagating in the environment towards the listeners who recover linguistic objects $\mathrm{O}_{\mathrm{L}}$. The

2014 success of communication is estimated by the Boolean variable $C^{\text {Env }}$. Bottom, all variables are 2015 internalized to provide a cognitive model of the communicating agent. 


\begin{tabular}{|c|c|c|}
\hline & $\begin{array}{l}\text { Production } \\
\text { infer } P(M \mid O)\end{array}$ & $\begin{array}{l}\text { Perception } \\
\text { infer } P(O \mid S)\end{array}$ \\
\hline $\begin{array}{l}\text { Motor theory } \\
\text { focus on } O_{S}\end{array}$ & $\underbrace{P\left(M \mid O_{S}\right)}_{\text {motor repertoire }}$ & $\propto \sum_{M}(\underbrace{P\left(M \mid O_{S}\right)}_{\text {motor decoder }} \underbrace{P(S \mid M)}_{\text {inverse model }})$ \\
\hline $\begin{array}{l}\text { Auditory theory } \\
\text { focus on } O_{L}\end{array}$ & $\propto P(M) \sum_{S}(\underbrace{P(S \mid M)}_{\text {direct model }} \underbrace{P\left(O_{L} \mid S\right)}_{\text {sensory targets }})$ & $\underbrace{P\left(O_{L} \mid S\right)}_{\text {sensory classifier }}$ \\
\hline $\begin{array}{l}\text { Perceptuo-motor } \\
\text { theory }\end{array}$ & $\propto P\left(M \mid\left[O_{S}=O_{L}\right]\right) \sum_{S}\left(P(S \mid M) P\left(O_{L} \mid S\right)\right)$ & $\propto P\left(\left[O_{L}=O_{S}\right] \mid S\right) \sum_{M}\left(P\left(M \mid O_{S}\right) P(S \mid M)\right)$ \\
\hline$C=$ True, i.e. $O_{S}=O_{L}$ & motor production sensory production & sensory perception \\
\hline
\end{tabular}

Figure 2. Probabilistic inferences for production and perception tasks instantiated within the

2019 framework of the motor, auditory and perceptuo-motor theories. The $\propto$ symbol denotes

2020 proportionality, i.e. to correctly obtain probability distributions, the expression shown has to

2021 be normalized. The denominations of the components of each equation refer to their possible

2022 interpretation in terms of cognitive processes:

2023 - sensory targets refer to the set of sensory distributions for each object (typically,

2024 "sensory" would be replaced by "auditory" in a basic auditory theory of speech

2025 perception, or possibly "audio-visual" in a modified version taking into account lip-

2026 reading: see note (1)),

2027 - motor repertoire refers to the set of motor distributions for each object,

2028 - $\quad$ sensory production refers to the distribution of sensory data (typically sounds) for each

2029 object,

2030 - motor production refers to the distribution of motor commands (typically articulatory

2031 gestures) for each object,

2032 - sensory classifier refers to the possibility of recovering the object from the stimulus

2033 input (typically the sound),

2034 - motor decoder refers to the possibility (thanks to the Bayesian summation) of

2035 recovering the object from the motor commands (or, in some variants of motor 
theories, the articulatory gesture),

- direct model refers to the possibility of predicting sensory information from the motor command (typically sound from the gesture),

- inverse model refers to the possibility of recovering the motor command from sensory information (typically the gesture from sound). 
Learning Agent

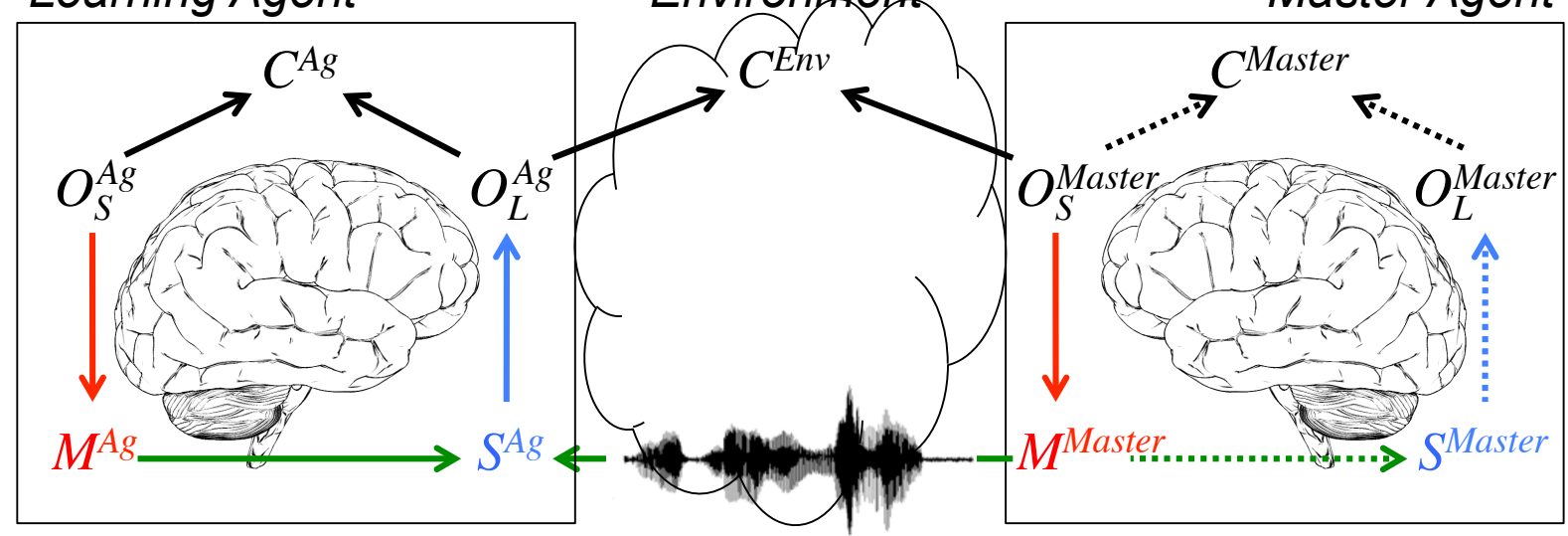

2044 Figure 3. Schema of the supervised learning scenario, where the Master Agent provides the

2045 Learning Agent with < object, stimulus> pairs.

2046

2047 

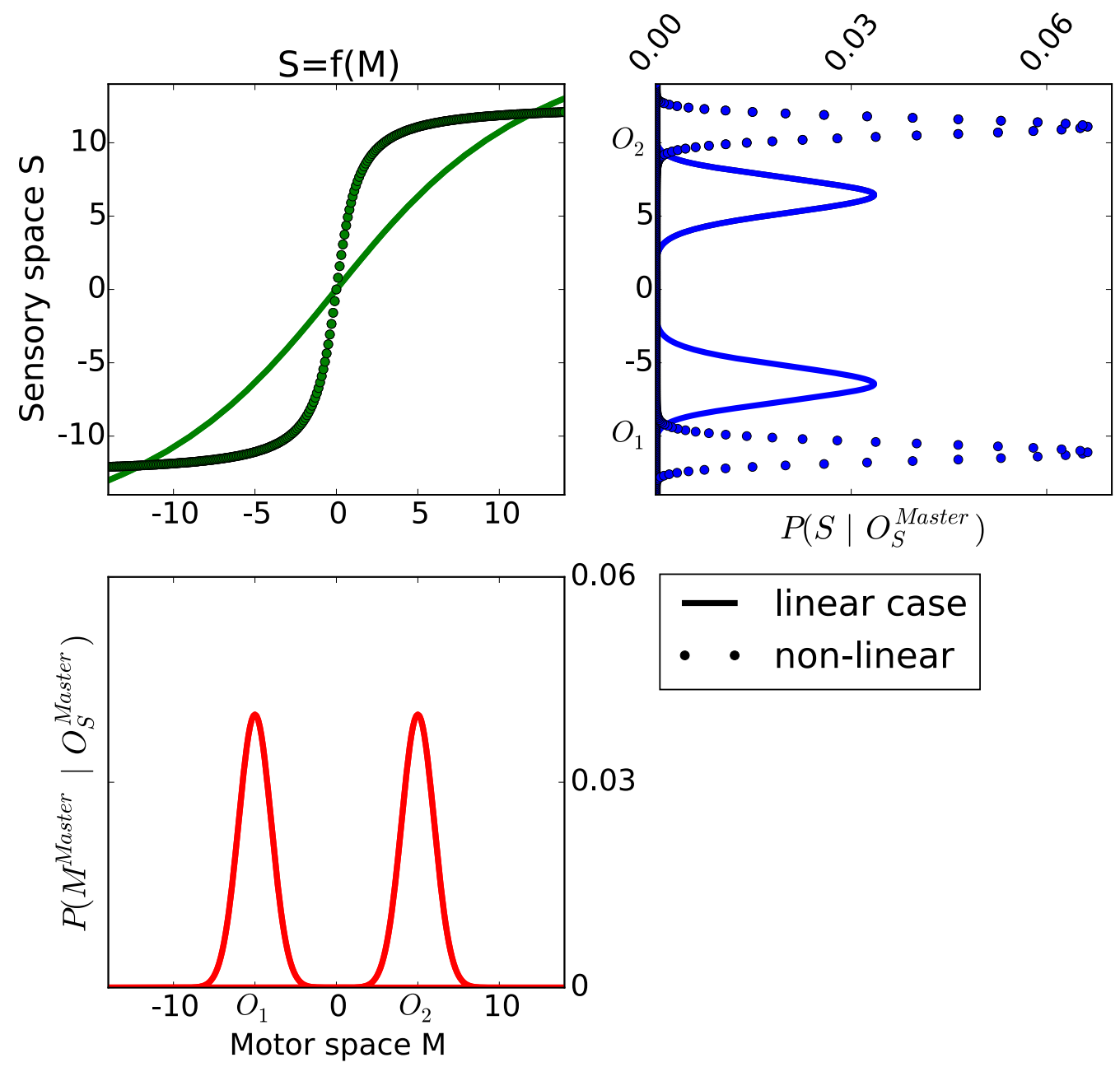

2048

2049 Figure 4. Summary of the stimulus production process of the Master Agent. Its motor

2050 repertoire is shown in the lower left panel. The model $f$ of the motor-to-sensory

2051 transformation is shown in the upper left panel, for two values of the nonlinearity parameter

2052 ( $a=0.01$ for the quasi-linear case and $a=0.1$ for the nonlinear case). The probability

2053 distributions of the resulting sensory inputs received by the Learning Agent are shown in the 2054 upper right panel. 

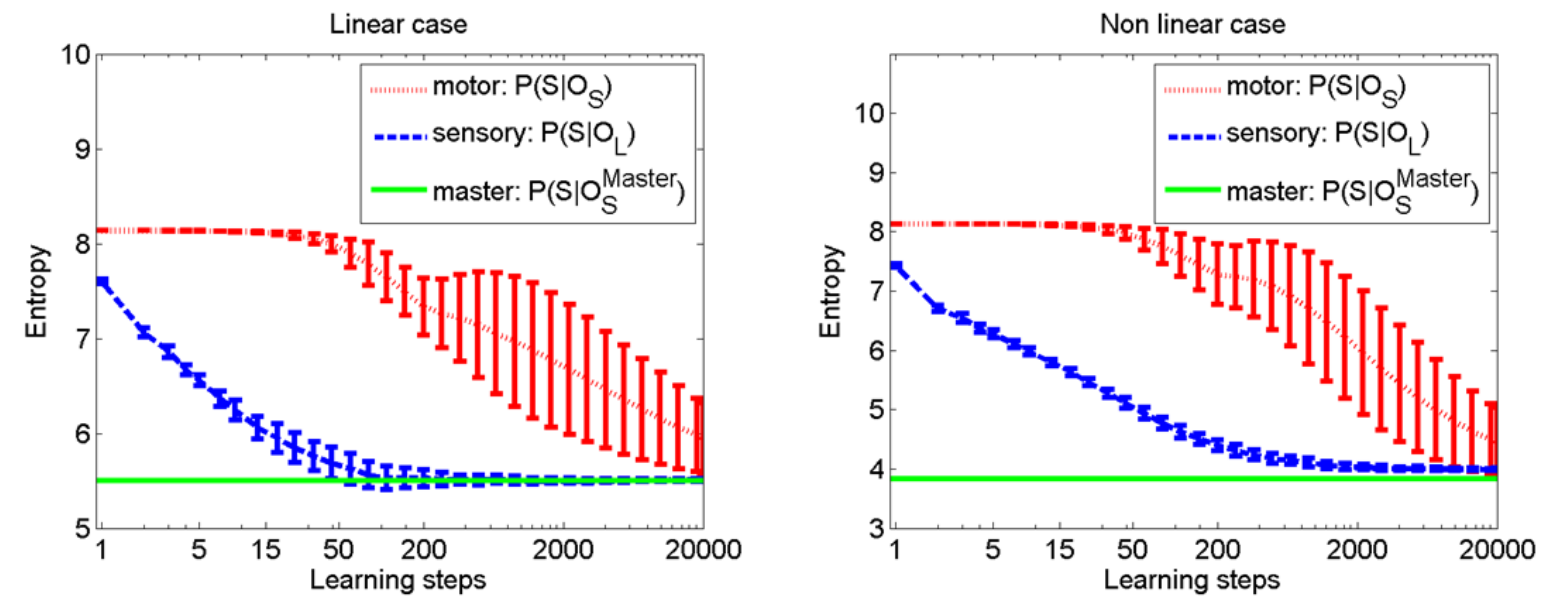

2058 Figure 5. Evolution of entropies of the sensory and motor models of the Learning Agents and

2059 production system of the Master Agent, as a function of the number of iterations of the

2060 learning algorithm, averaged over the possible object values. Left column: linear case; right

2061 column: nonlinear case. In each case, 12 different simulations were run, corresponding to

2062 random initializations of the learning process. The standard deviations shown are computed

2063 over these 12 different simulations. 


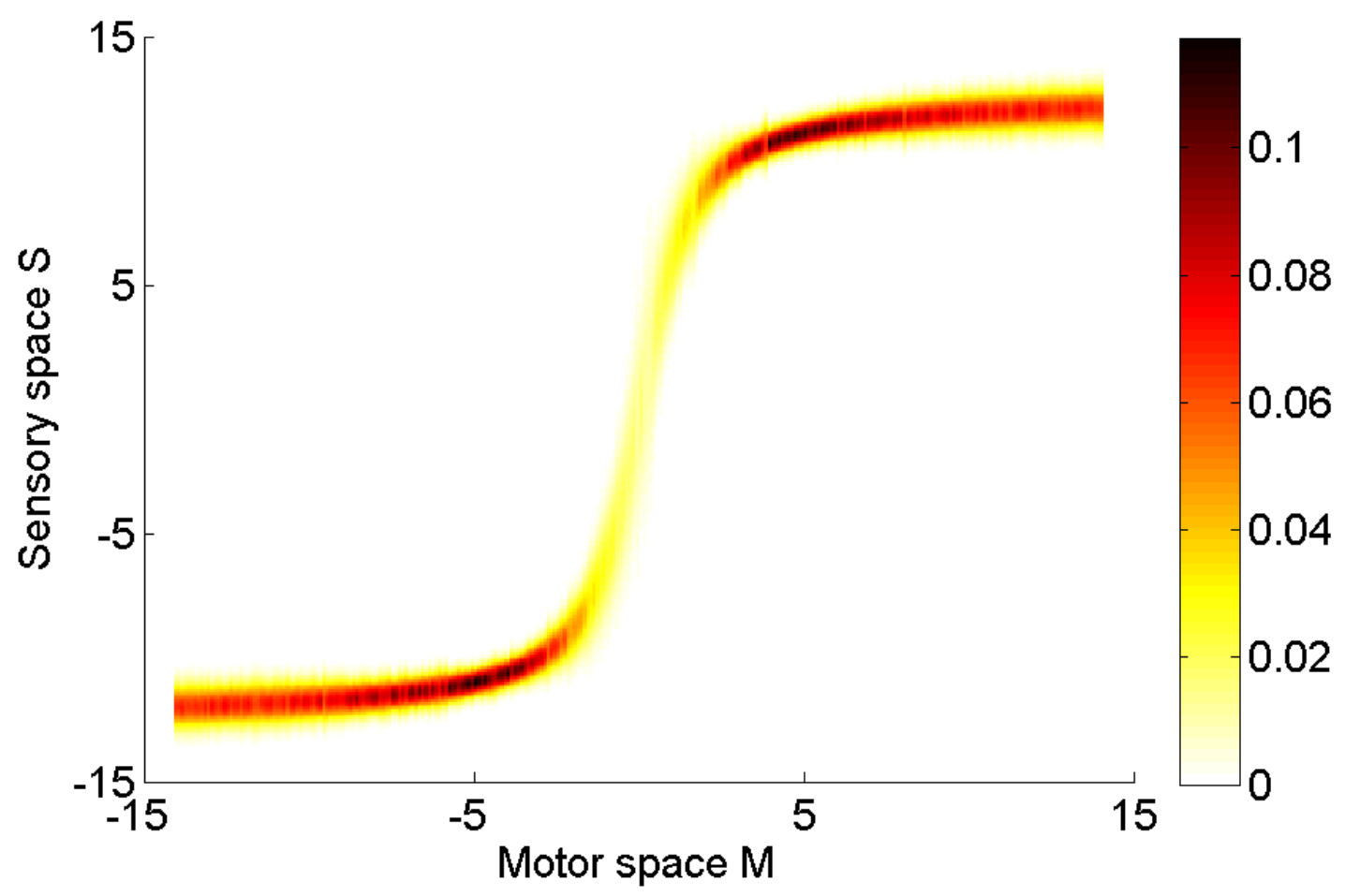

2065 Figure 6. An instance of the learned internal model of the motor-to-sensory transformation, 2066 after 20,000 learning iterations, in a nonlinear setting $(a=0.1)$. For each motor gesture $m$ of 2067 the $\mathrm{x}$-axis, the probability distribution over resulting sensory stimulus $P(S \mid[M=m])$ is read 2068 vertically, with the color code indicating probability (white to yellow to red to black color2069 map (light gray to black), in order of increasing probability value). Black regions (resp.

2070 yellow/light gray) therefore correspond to low-variance (resp. high variance) Gaussian 2071 probability distributions, that is to say, well-explored (resp. poorly explored) portions of the 2072 motor-to-sensory transformation. 


\section{Linear case}
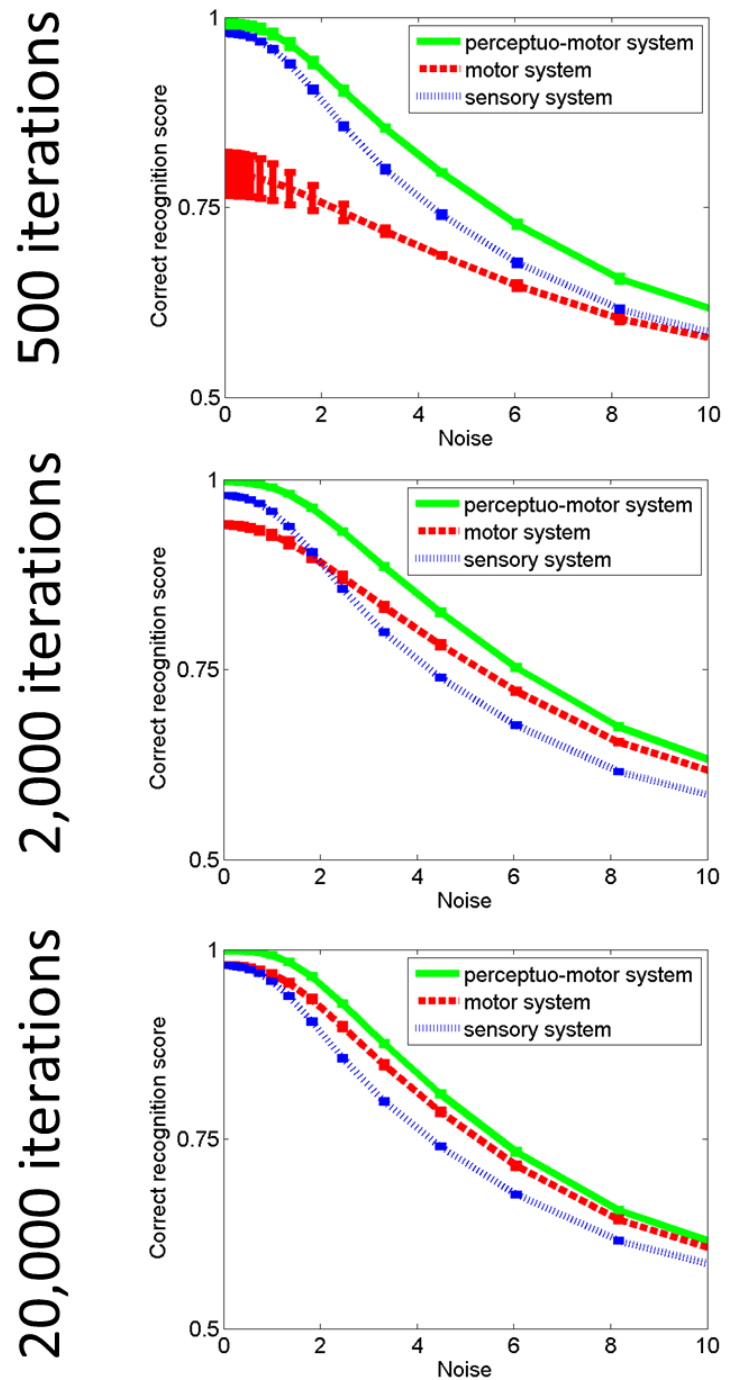

Non-linear case
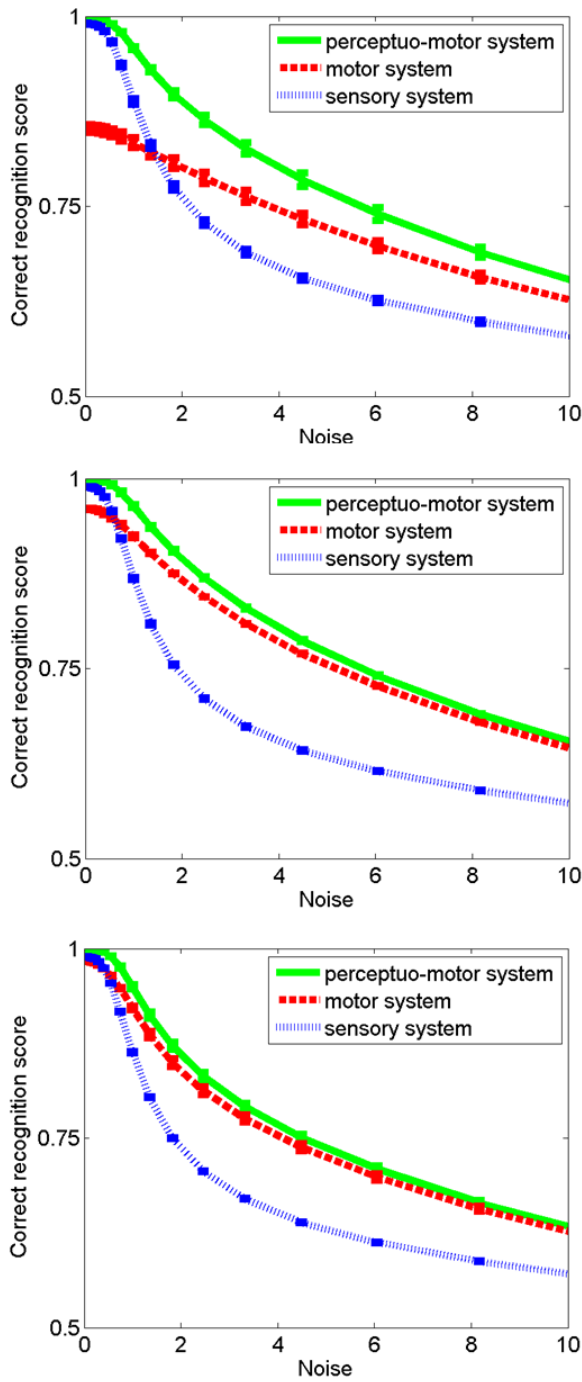

Figure 7. Evolution of correct recognition scores of motor, sensory and perceptuo-motor

2076 models of perception, as a function of environment noise. In each case, 12 different

2077 simulations were run, corresponding to random initializations of the learning process. The standard deviations shown are computed over these 12 different simulations. 


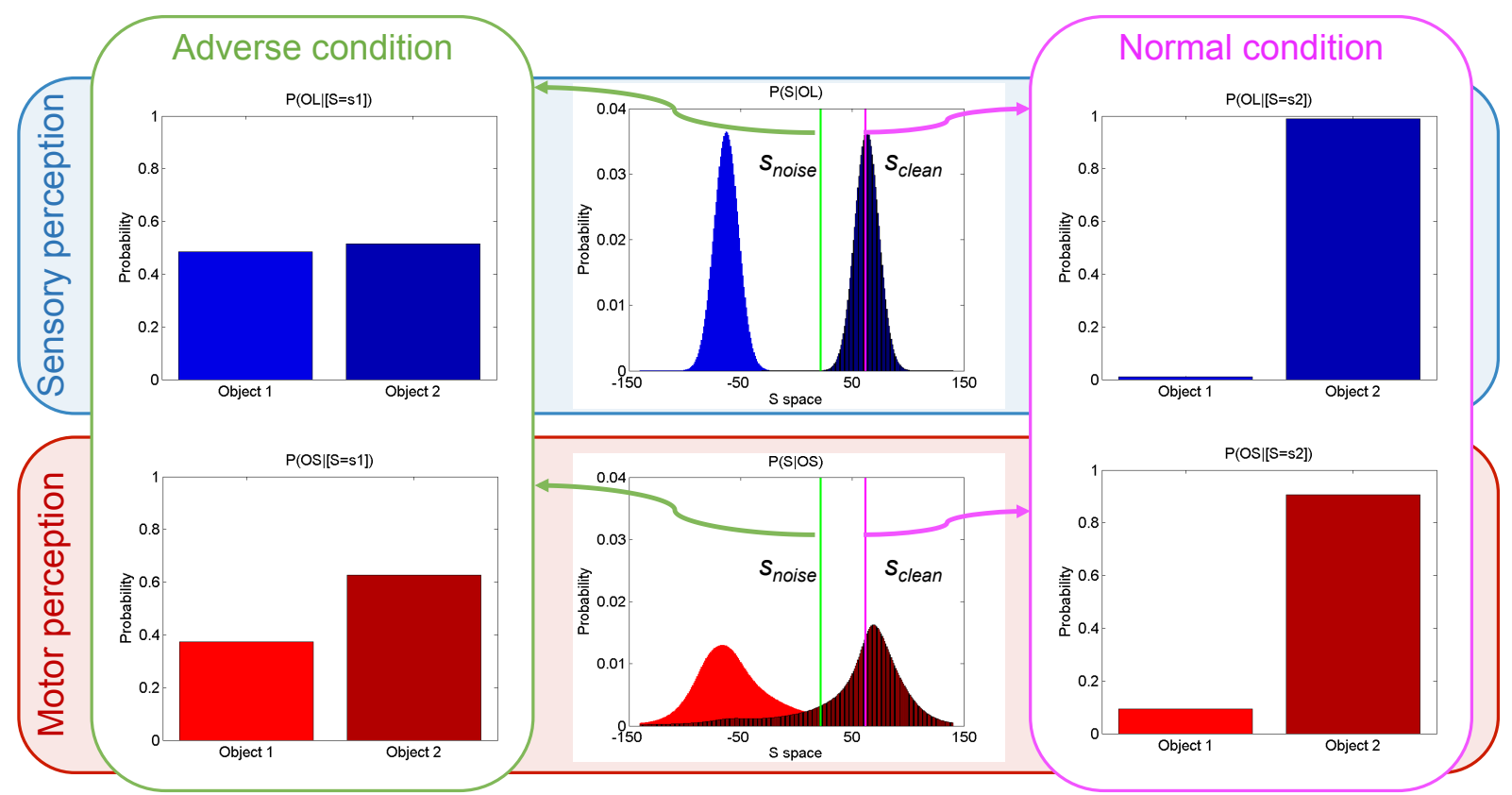

2081 Figure 8. Illustration (linear case, after 1,200 learning iterations) of sensory (top row) and

2082 motor (bottom row) categorization processes on example stimuli $s_{\text {noise }}$ in adverse conditions

2083 (left column) and $s_{\text {clean }}$ in normal conditions (right column), as probabilistic inference from 2084 learned prototypes (center column). 

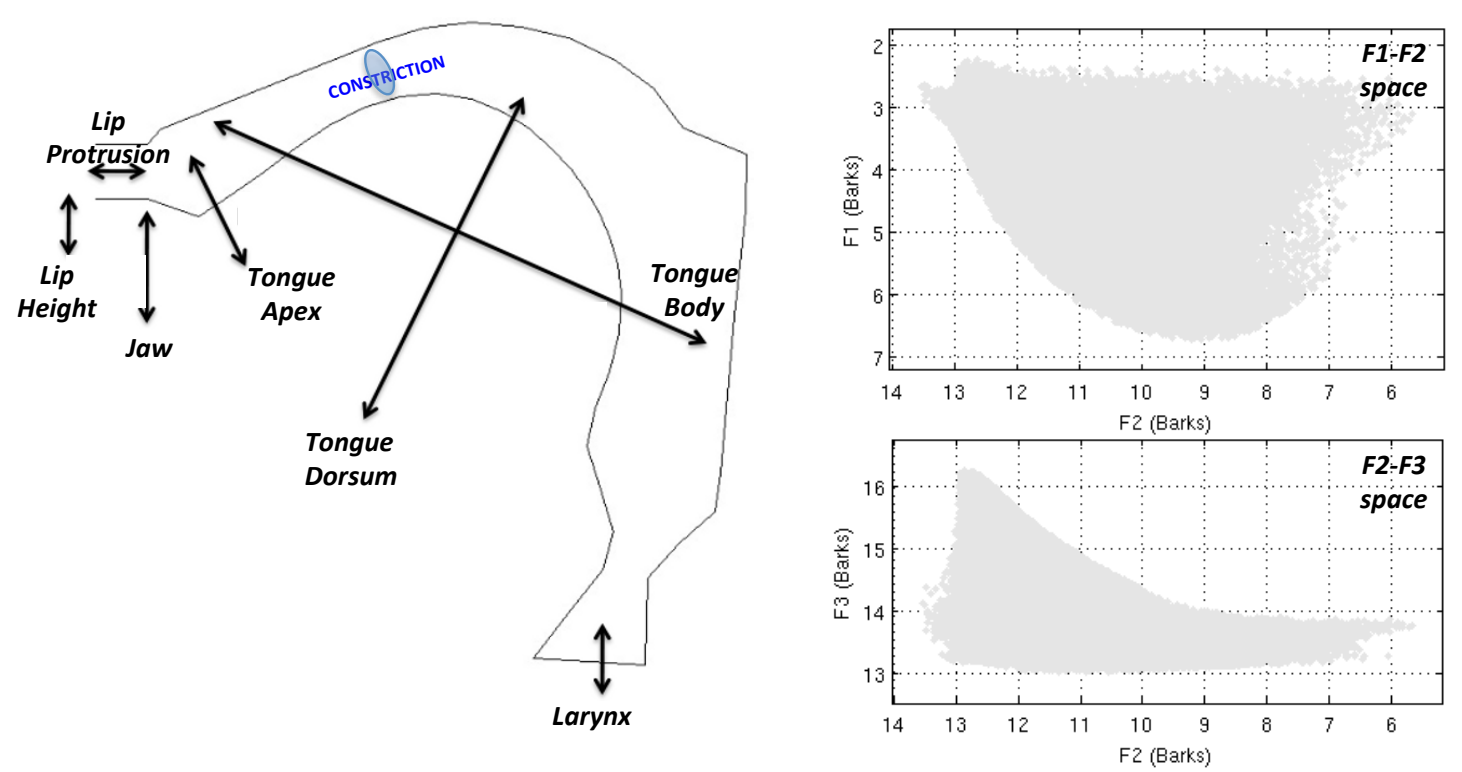

Figure 9. The vocal tract VLAM model. Left: the seven articulatory parameters (Jaw, Lip

2089 Height and Protrusion, Tongue Body, Dorsum and Apex, and Larynx) enable the vocal tract

2090 shape to be driven. The Constriction is defined by the position where the vocal tract area is

2091 minimum. Vowels are constrained to have an area greater than $0.15 \mathrm{~cm}^{2}$. Plosives are

2092 constrained to between 0.05 and $0.15 \mathrm{~cm}^{2}$. Right: plots of the regions of the acoustic space

2093 (top: $(F 1, F 2)$ plane, bottom: $(F 2, F 3)$ plane) that result from articulatory configurations in

2094 VLAM. 

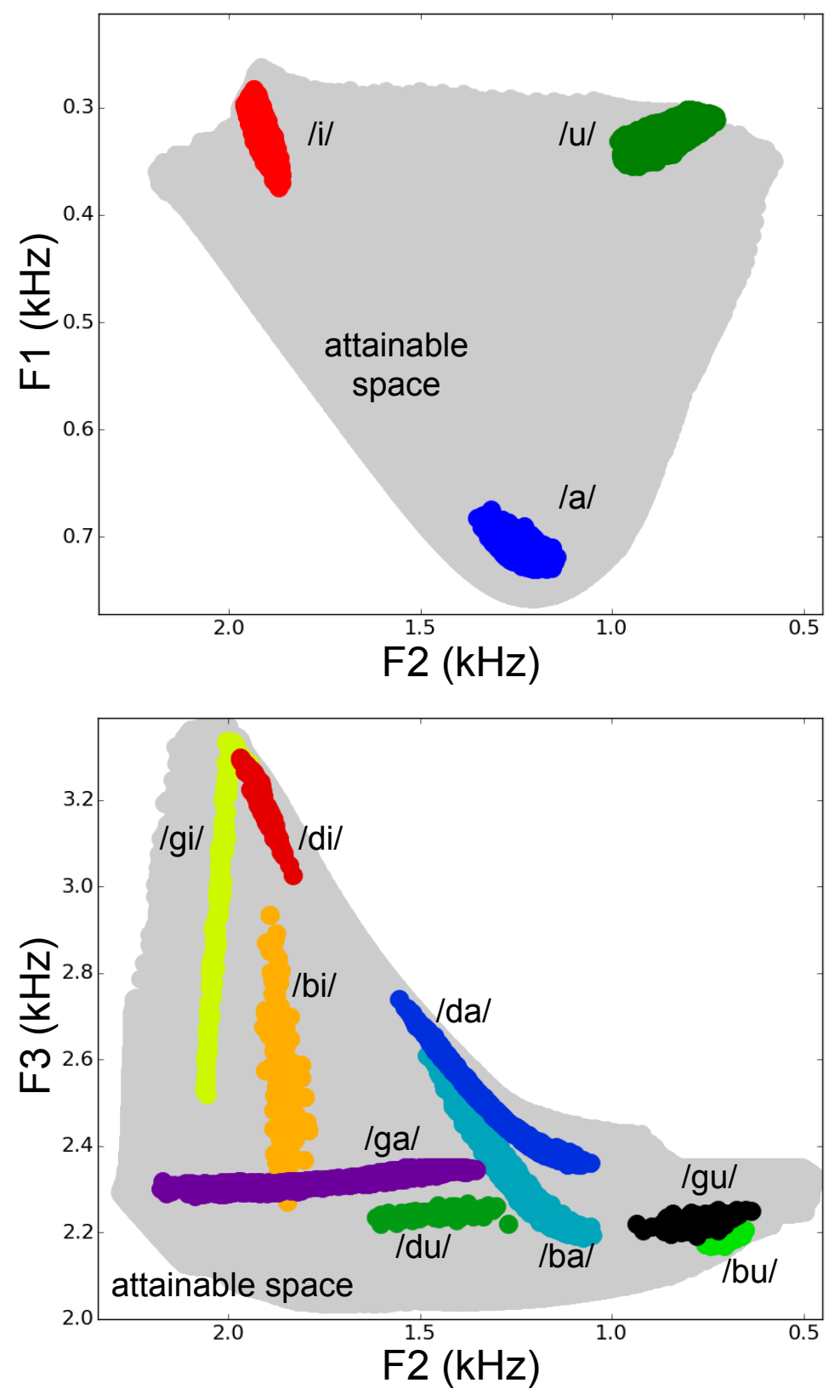

2096

2097 Figure 10. Synthetic syllables in acoustic space. Top: $(F 2, F 1)$ for vowels. Bottom: $(F 2, F 3)$ 2098 for plosives in each syllabic context. 


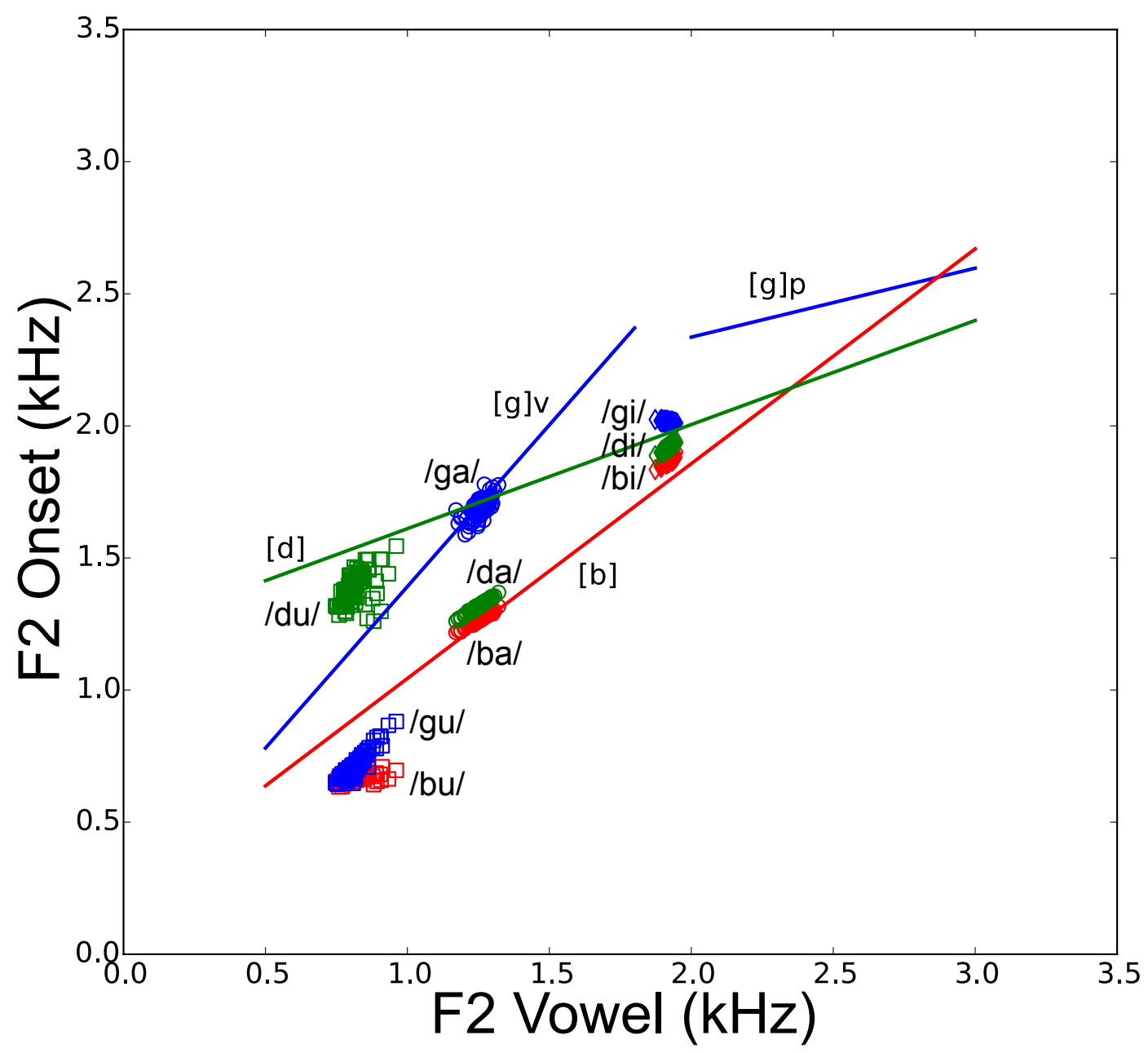

Figure 11. Locus displays for VLAM simulations (color marks annotated with /ba/, /bi/, /bu/, etc.) compared with locus equations provided by Sussman (1998) (portions of straight lines annotated with [d], [b], etc.). For VLAM simulations, each mark is displayed at the position corresponding to the $F 2$ value for the vowel on the $\mathrm{x}$-axis, and the $F 2$ value for the consonant on the y-axis. Sussman's locus equations are derived by pooling the same frequency coordinates for natural utterances from 20 American English speakers (see Sussman, 1998, Fig. 5). Sussman provides one equation for $/ \mathrm{b} /$, one for $/ \mathrm{d} /$ and two separate equations for $/ \mathrm{g} /$ : one when the context vowel is front and the plosive is therefore palatal, and another one when the context vowel is back and the plosive is therefore velar. 


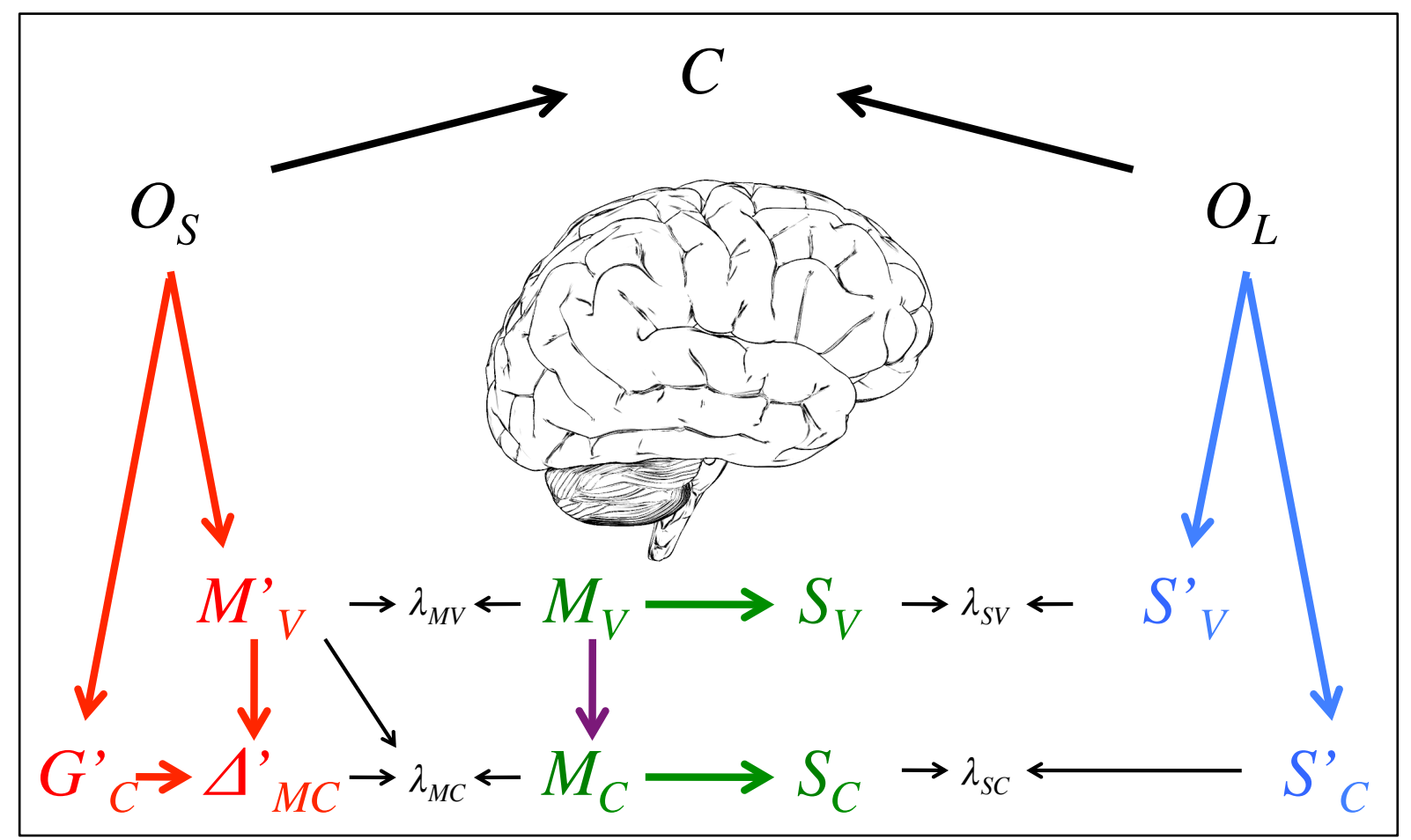

$P\left(O_{S} G_{C}^{\prime} M_{V}^{\prime} \Delta_{M C}^{\prime} \lambda_{M V} \lambda_{M C} M_{V} M_{C} S_{V} S_{C} \lambda_{S V} \lambda_{S C} S_{V}^{\prime} S_{C}^{\prime} O_{L} C\right)=$ $P\left(O_{S}\right) P\left(M_{V}^{\prime} \mid O_{S}\right) P\left(G_{C}^{\prime} \mid O_{S}\right) P\left(\Delta_{M C}^{\prime} \mid M_{V}^{\prime} G_{C}^{\prime}\right)$

$P\left(\lambda_{M V} \mid M_{V}^{\prime} M_{V}\right) P\left(\lambda_{M C} \mid M_{V}^{\prime} \Delta_{M C}^{\prime} M_{C}\right)$

$P\left(M_{V}\right) P\left(S_{V} \mid M_{V}\right) P\left(M_{C} \mid M_{V}\right) P\left(S_{C} \mid M_{C}\right)$

$P\left(\lambda_{S V} \mid S_{V} S_{V}^{\prime}\right) P\left(\lambda_{S C} \mid S_{C} S_{C}^{\prime}\right)$

$P\left(O_{L}\right) P\left(S_{V}^{\prime} S_{C}^{\prime} \mid O_{L}\right)$

2111 Figure 12. The COSMO-S model for processing syllables. This is illustrated by a graphical

2112 representation (Top), and by the decomposition of its joint probability distribution as a

2113 product of probabilistic terms (Bottom). In red (left part), the motor system, in green (middle

2114 part), the sensory-motor system, in blue (right part), the auditory system. 


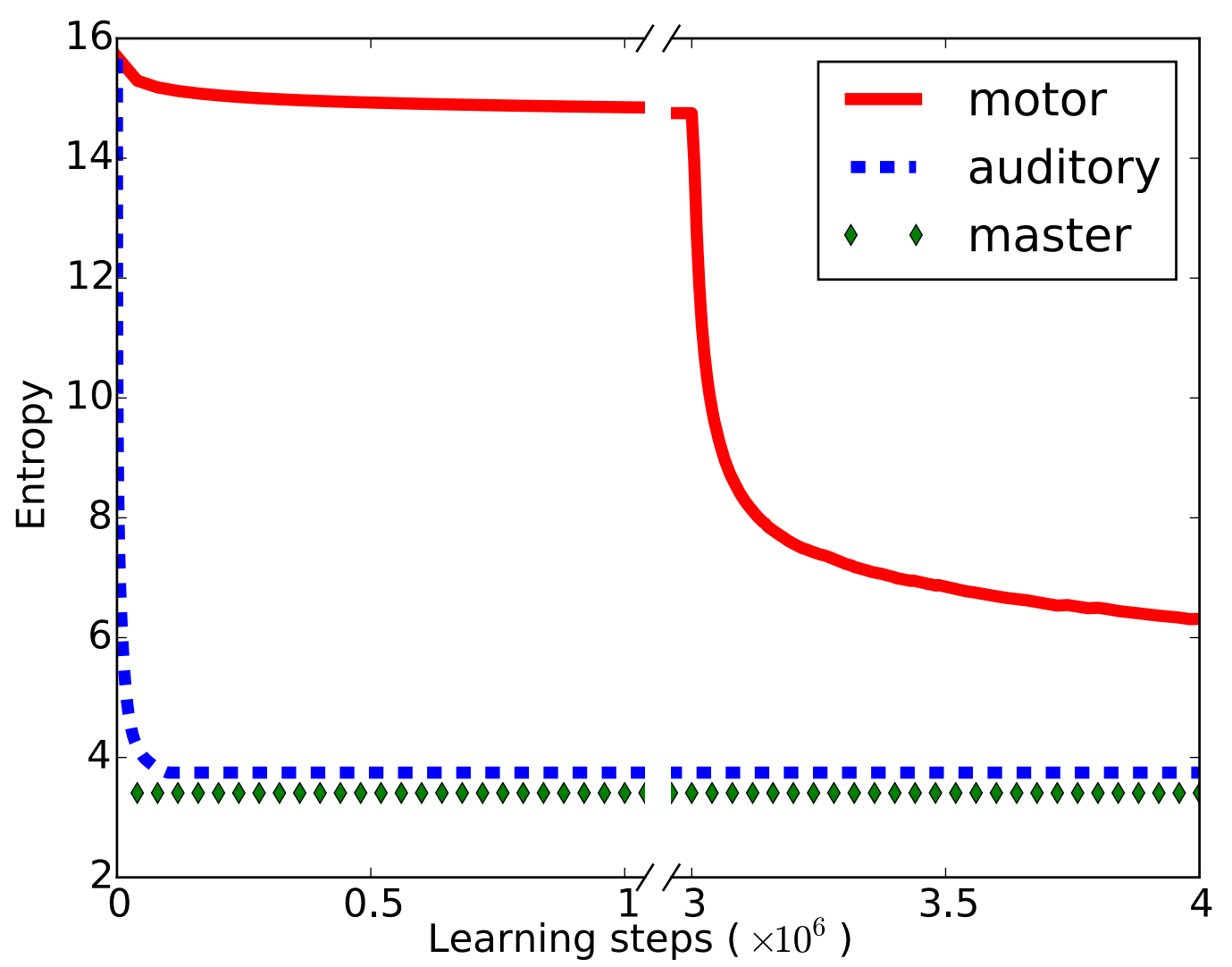

2117 Figure 13. Evolution of entropies of the auditory and motor models of the Learning Agents 2118 and production system of the Master Agent, as a function of the number of iterations of the 2119 learning algorithm, averaged over the possible object values, in the syllable experiment. For 2120 the auditory model, learning corresponds to the sensory learning phase L1 with 4,000,000

2121 iterations. For the motor model, learning starts with the sensory-motor learning phase L2 with $21223,000,000$ iterations followed by the motor learning phase L3 from 3,000,000 to 4,000,000 2123 iterations. 

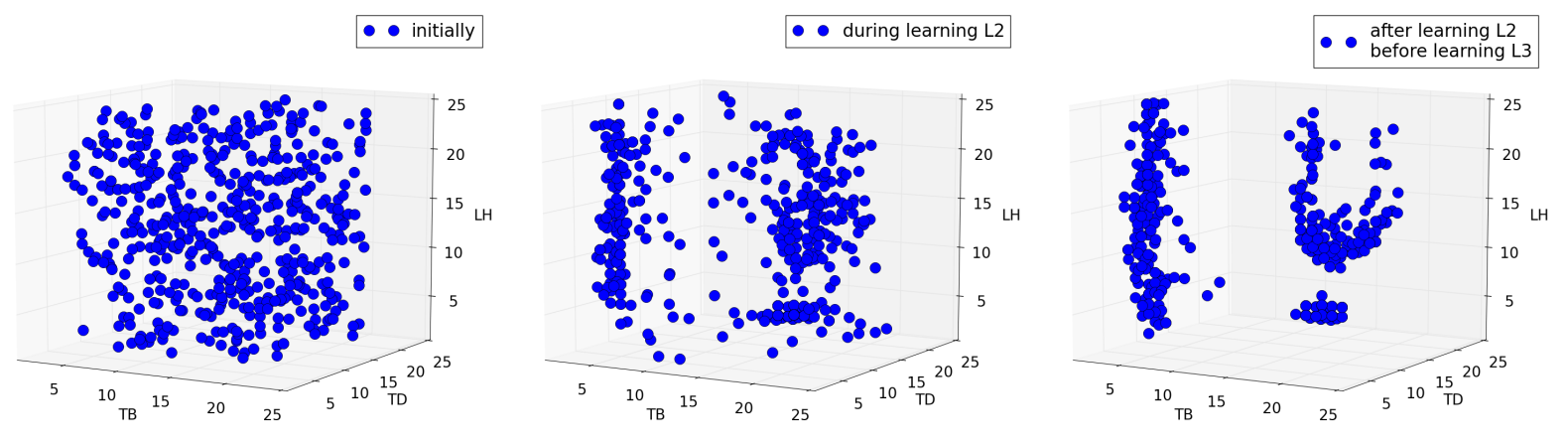

$\bullet$ during learning L3
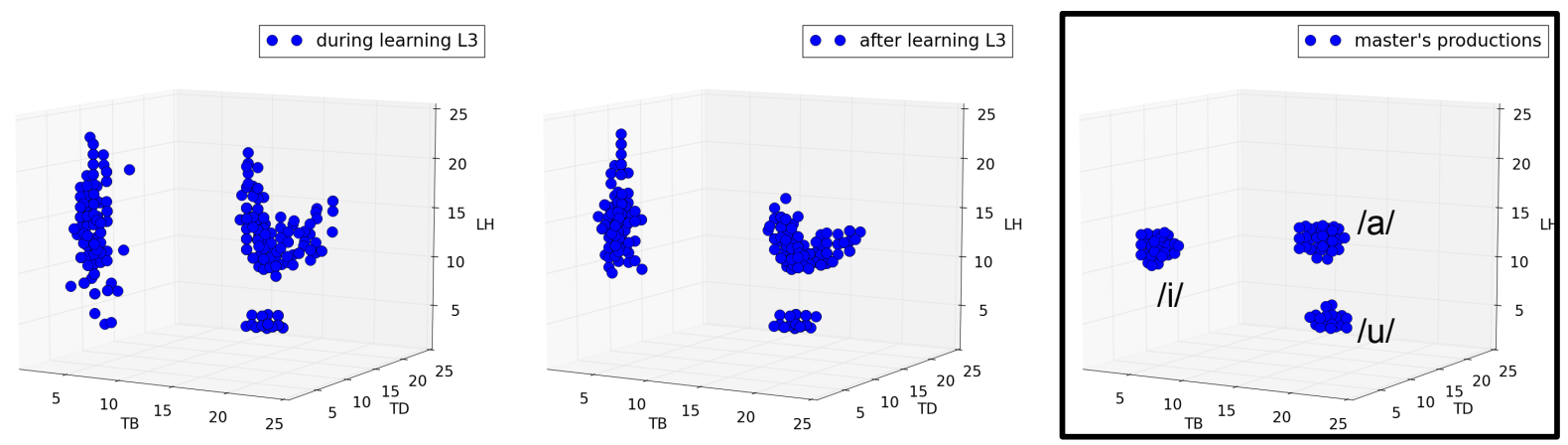

2125 Figure 14. Illustrating exploration in the motor space in COSMO-S. Each graph displays

2126 samples from the probability distributions $P\left(M_{V}^{\prime A g} \mid\left[S_{V}^{A g}=s_{v}\right]\left[O_{S}^{A g}=o_{S}\right]\left[\lambda_{M V}^{A g}=1\right]\right)$ in the

2127 three-dimensional space TB (Tongue Body), TD (Tongue Dorsum) and LH (Lip Height), with

$2128 s_{v}$ the vowel acoustic target and $o_{S}$ the corresponding syllable label. Motor variables are

2129 specified by normalized values between 0 and 25. Each panel shows 500 samples taken at 500

2130 successive time-steps (one sample per learning iteration), during five stages of the exploration

2131 process (see caption of each panel). The bottom right panel shows, for comparison, the motor

2132 distribution of the Master Agent. 

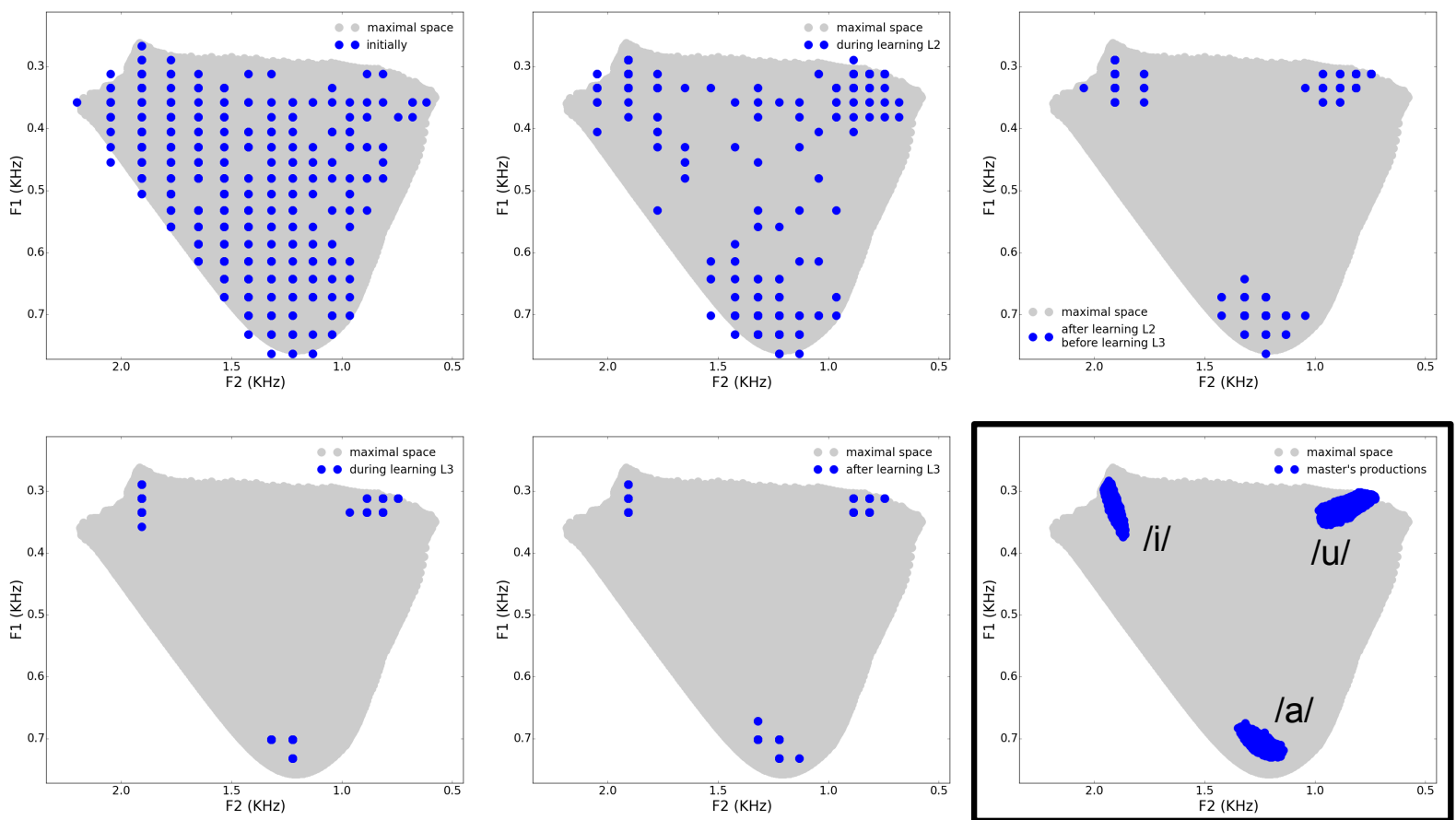

Figure 15. Illustrations of the exploration in the vowel space in COSMO-S. Each graph

2136 displays the images in the acoustic $(F 2, F 1)$ plane of the exact same motor samples as in

2137 Figure 14, via the articulatory-to-acoustic transformation. Each panel concerns the same five

2138 stages of the exploration process as in Figure 14. For comparison, the bottom right panel

2139 shows the stimulus distribution of the Master Agent. 

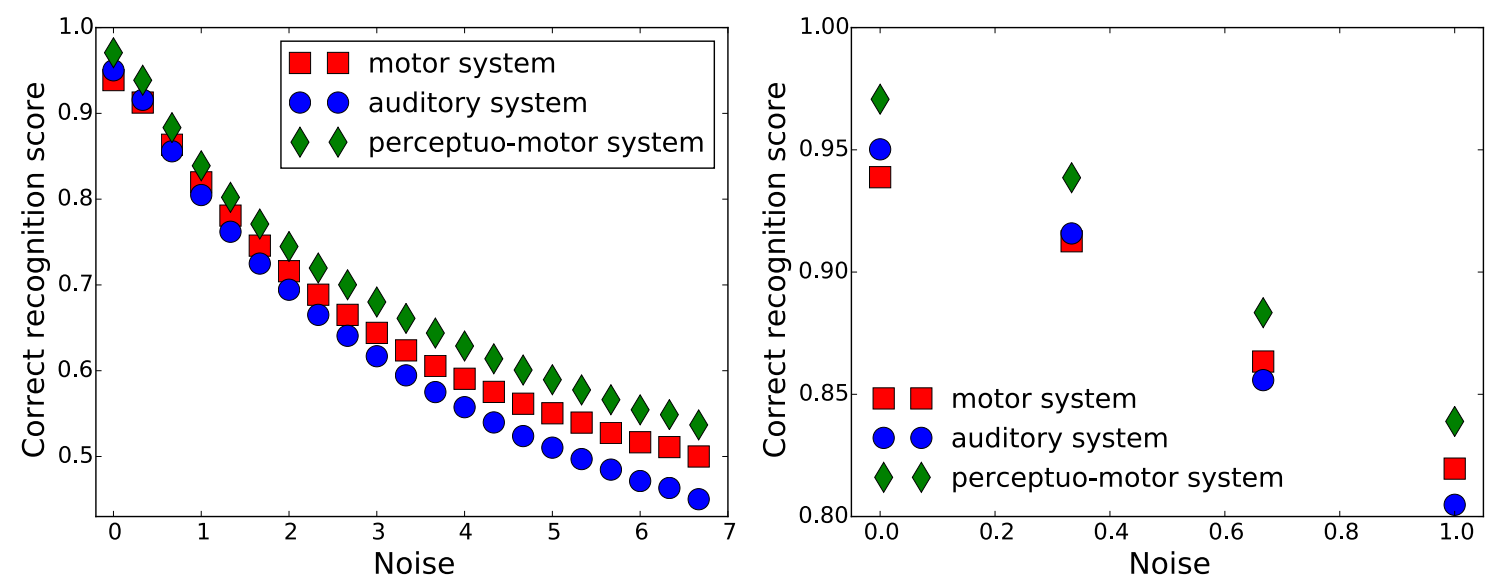

2142 Figure 16. Results of the classification process for syllables presented at various levels of

2143 noise. The correct recognition rates for the auditory, motor and perceptuo-motor

2144 implementations of the perception task in the COSMO-S model are displayed. Right plot:

2145 zoom of the left plot at low levels of noise highlights the inversion of performance between

2146 the auditory system (better under normal conditions) and the motor system (better at noisy 2147 conditions). 

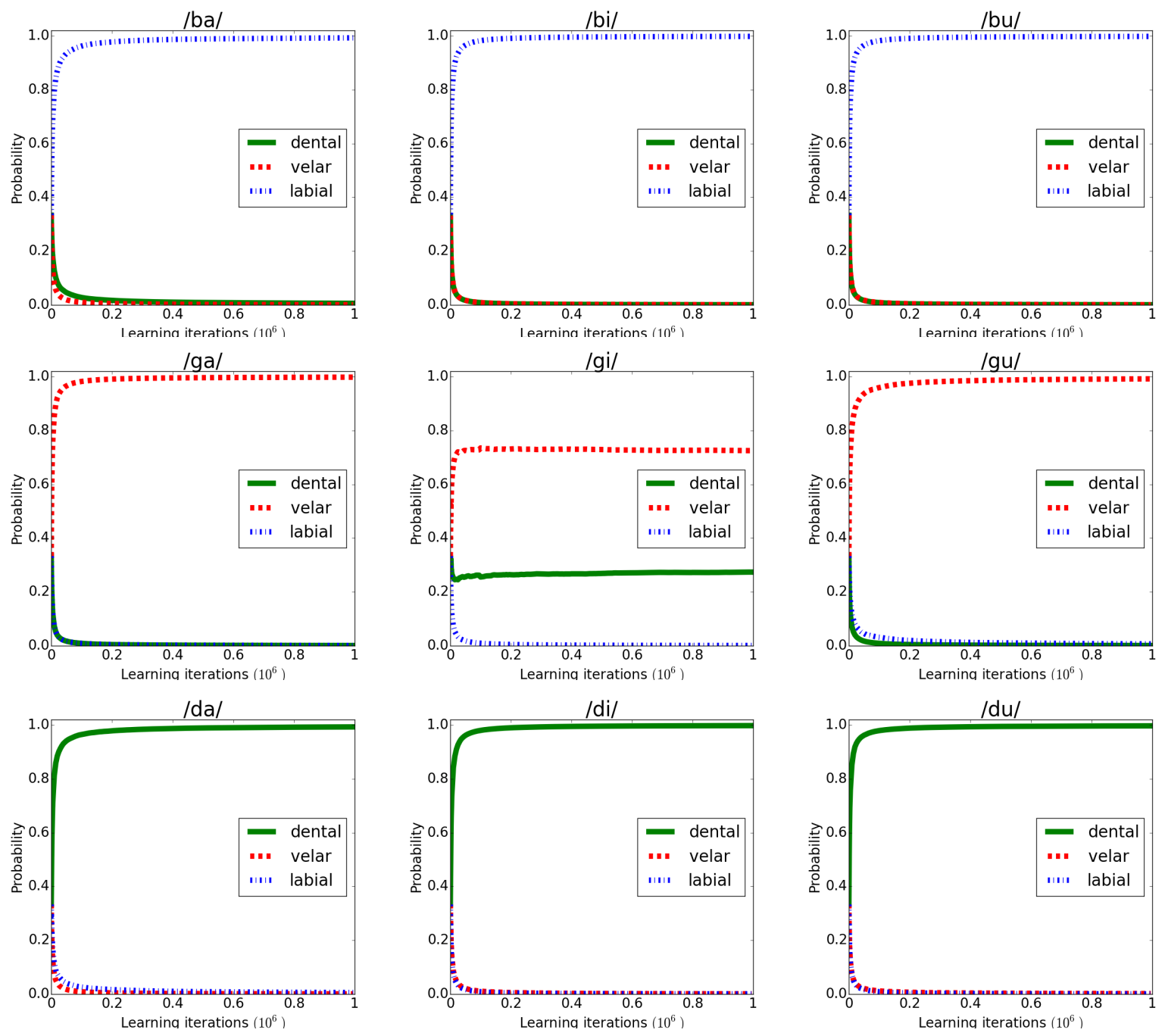

2150 Figure 17. Learning the place of articulation for plosives. Evolution of the probabilities of the

2151 motor variable $P\left(G_{C}^{\prime A g} \mid O_{S}^{A g}\right)$ with the number of iterations in learning, for the 9 objects $O_{S}^{A g}$

2152 (see text). 

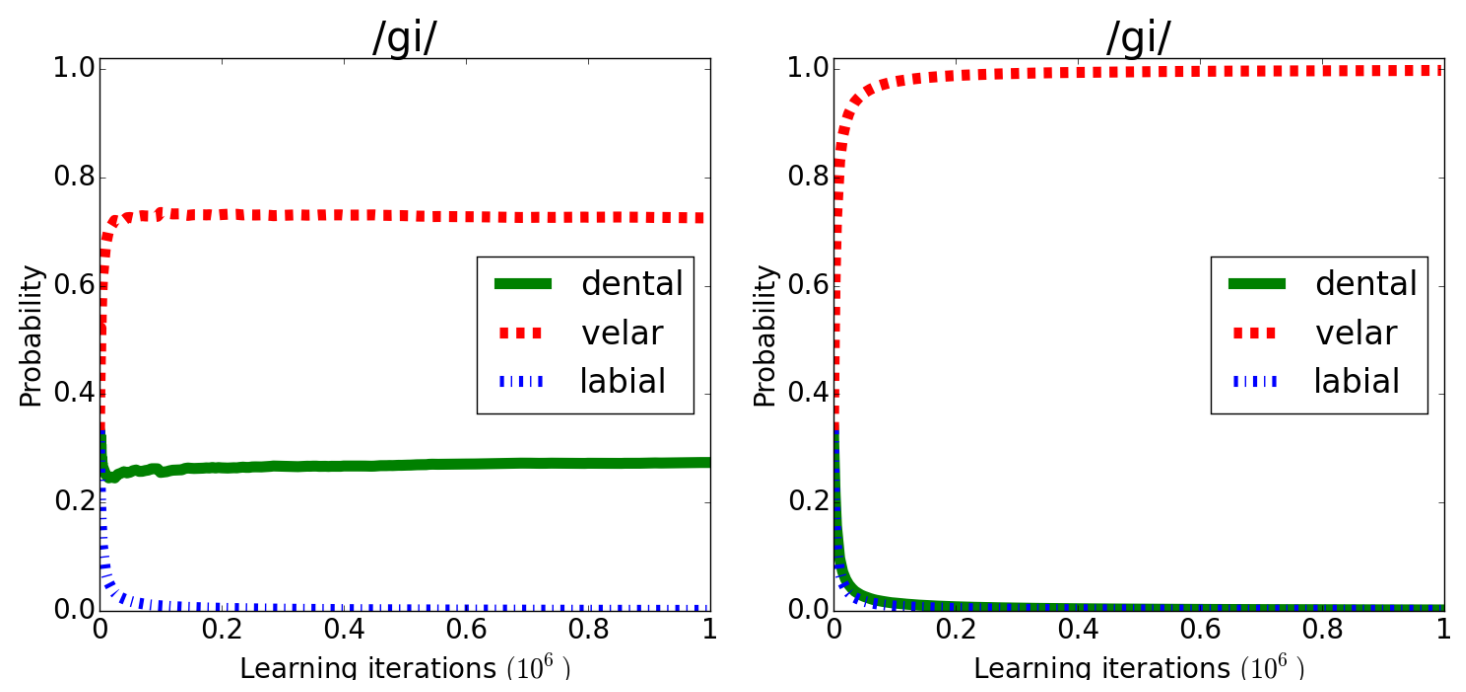

2155 Figure 18. The role of hyperarticulation in the emergence of phonological categories.

2156 Evolution of the probabilities of the motor variable $P\left(G_{C}^{\prime A g} \mid O_{S}^{A g}\right)$ with the number of

2157 iterations in learning, for the object $O_{S}^{A g}=/ g i /$, comparing the cases where learning is

2158 without (left, identical to the middle panel of Figure 16) or with (right) hyper-articulation by 2159 the Master Agent (see text). 